University of Zurich

Department of Economics

Working Paper Series

ISSN 1664-7041 (print)

ISSN 1664-705X (online)

Working Paper No. 358

\title{
Killer Acquisitions and Beyond: Policy Effects on Innovation Strategies
}

Igor Letina, Armin Schmutzler and Regina Seibel

First version: February 2020

This version: October 2021 


\title{
Killer Acquisitions and Beyond: Policy Effects on Innovation Strategies
}

\author{
Igor Letina, Armin Schmutzler and Regina Seibel*
}

This version: October 2021

First version: February 2020

\begin{abstract}
This paper provides a theory of strategic innovation project choice by incumbents and start-ups which serves as a foundation for the analysis of acquisition policy. We show that prohibiting acquisitions has a weakly negative innovation effect. We provide conditions determining the size of the effect and, in particular, conditions under which it is zero. We further analyze the effects of less restrictive policies, including merger remedies and the tax treatment of acquisitions and initial public offerings. Such interventions tend to prevent acquisitions only if the entrant has sufficiently high stand-alone profits.
\end{abstract}

Keywords: innovation, killer acquisitions, merger policy, potential competition, startups.

JEL: O31, L41, G34

${ }^{*}$ Letina: Department of Economics, University of Bern and CEPR. Schmutzler: Department of Economics, University of Zurich and CEPR. Seibel: Department of Economics, University of Zurich. Email: igor.letina@vwi.unibe.ch, armin.schmutzler@econ.uzh.ch, and regina.seibel@econ.uzh.ch. We are grateful for useful discussions to Alessandra Allocca, Philipp Brunner, Steve Callander, Florian Ederer, Marc Möller, Joao Montez, José L. Moragá-Gonzalez, Christian Oertel, Nicolas Schutz, Yossi Spiegel, Konrad Stahl, Xavier Vives and seminar participants at Bar-Ilan University, CEPR joint IMO and ENT Conference, CRESSE 2021, Carlos III Madrid, EARIE 2021, Econometric Society World Congress 2020, Higher School of Economics, IIOC 2021, National University of Singapore, New Economic School, Paris School of Economics, Paris Seminar on the Economics of Digitization, Swiss Society of Economics and Statistics 2021, Verein für Socialpolitik (IO Panel) and Universities of Basel, British Columbia, Groningen, Hamburg, Lausanne, Leicester, Linz, Mannheim (MaCCI 2020), Milano (Cattolica), St. Gallen and Zurich. 


\section{Introduction}

Mergers rarely trigger interventions by competition authorities unless they involve substantial additions of incumbent market shares. Recently, practitioners and academics have argued that this lenient approach to merger control may be flawed, as mergers between firms that are not currently competing might eliminate potential competition. ${ }^{1}$ This issue even arises when "the target firm has no explicit or immediate plans to challenge the incumbent firm on its home turf, but is one of several firms that is best placed to do so in the next several years" (Shapiro, 2018). It becomes more pressing when the acquiree's technology would otherwise enable it to compete against the incumbent in the near future.

Such concerns arise in various sectors. For instance, in the digital economy, Google, Amazon, Apple, Facebook and Microsoft bought start-ups worth a total of 31.6 billion USD in 2017. ${ }^{2}$ Google acquired about one firm per month between 2001 and 2020. ${ }^{3}$ A possible reason may be that the acquiring firms are better at commercializing the ideas of the start-ups, so that an acquisition may be efficient. Recent evidence suggests, however, that anti-competitive motives may also be important. The work of Cunningham, Ederer and Ma (2021) for the pharmaceutical industry is a compelling case in point. The authors show that incumbent firms often engage in so-called killer acquisitions by purchasing start-ups with the sole purpose of eliminating potential competition, without intending to commercialize the innovation. ${ }^{4}$ Even when incumbents do commercialize the innovation, acquisitions need not be innocuous, as they may widen the technological lead of a dominant incumbent, making entry ever harder (e.g. Bryan and Hovenkamp, 2020a).

These considerations suggest rethinking the predominant practice of waving through acquisitions of small innovative start-ups by incumbent firms. ${ }^{5}$ There appears to be a

\footnotetext{
${ }^{1}$ This concern is reflected in policy reports such as Crémer, de Montjoye and Schweitzer (2019) ("EU Report"), Furman, Coyle, Fletcher, McAuley and Marsden (2019) ("Furman Report") or Scott Morton, Bouvier, Ezrachi, Jullien, Katz, Kimmelman, Melamed and Morgenstern (2019) ("Stigler Report"); see also Salop (2016), Salop and Shapiro (2017), Hovenkamp and Shapiro (2017), Bryan and Hovenkamp (2020b).

${ }^{2}$ See The Economist 26/10/2018 "American tech giants are making life tough for start-ups". Examples include Facebook's takeovers of WhatsApp, Instagram and Oculus CR, Google's acquisition of DoubleClick, Waze and YouTube, and Microsoft's purchases of GitHub and LinkedIn. For more descriptive statistics on start-up acquisitions, see Gautier and Lamesch (2020).

${ }^{3}$ https://en.wikipedia.org/wiki/List_of_mergers_and_acquisitions_by_Alphabet

${ }^{4}$ The use of the "killer" metaphor in the literature is not uniform. Some authors apply the expression "kill zone" to start-up activities that are so close to those of dominant incumbents that they may trigger hostile behavior towards the entrant, without implying that the incumbent would not commercialize the start-up's technologies.

${ }^{5} \mathrm{~A}$ rare exception was the FTC's intervention against the acquisition of HeartWare by Thoratec, a maker of left ventricular assist devices, in 2009 on the grounds that "HeartWare alone represents a significant threat to Thoratec's LVAD monopoly;" see https://www.ftc.gov/sites/default/files/documents/ cases/2009/07/090730thorateadminccmpt.pdf. More recently, the biotech firm Illumina abandoned its proposed acquisition of the small rival Pacific Biosciences following opposition of the U.S. FTC and the U.K. CMA. The former explicitly referred to the extinction of Pacific Biosciences as a "nascent competitive threat". For similar reasons, the FTC imposed a divestiture before approving the acquisition of College
} 
broad consensus among economists that this approach is excessively lenient. That said, a per-se prohibition of start-up acquisitions would not be desirable either: As many observers have pointed out, the prospect of selling the firm may increase the entrant's ex-ante innovation incentive, even without commercialization by the incumbent. ${ }^{6}$ Since Rasmusen (1988), several academic papers have formalized this "entry-for-buyout logic". However, the option of buying an innovative entrant could reduce innovation efforts of the incumbent. Therefore, the overall effect of a prohibition of acquisitions on innovation is unclear ex ante and should be expected to depend on market characteristics. This suggests a selective approach towards treating start-up acquisitions, where the competition authority intervenes only in markets where the benefits from preserving potential competition outweigh any possible negative innovation effects.

Our paper focuses on the innovation effects of acquisition policy. Its purpose is twofold. First, we identify conditions justifying a prohibition of acquisitions. To this end, we show that, though the innovation effect of forbidding acquisitions is weakly negative, its size depends on market characteristics. In particular, we identify circumstances under which the effect is absent, so that the standard pro-competitive arguments suffice to justify a prohibition. Second, as one might argue that selective intervention is difficult to implement in practice, we also analyze the effects of several policy instruments which leave the acquisition decision to the firms, but influence acquisition incentives. Specifically, we consider merger remedies, acquisition taxes and preferential treatment of initial public offerings.

Our model is generic rather than tailored to any single industry, as we do not impose any functional form on demand or profit and we do not restrict attention to either process or product innovations. It is based on a novel theory of $R \& D$ project choice of incumbents and start-ups that enables us to study project variety and duplication. An incumbent monopolist possesses a technology that allows her to operate in a product market without innovation. By contrast, an entrant has to innovate in order to produce. Contrary to most papers in the innovation literature, which only analyze the overall level of $R \& D$ spending, we allow firms to strategically choose in which innovation projects to invest as well as how much to invest in each project. Such a representation captures important aspects of many real-world innovation decisions. ${ }^{7}$ Ex ante, projects only differ with respect to investment costs; ex post, only one project will lead to an innovation. With some probability, this innovation will be drastic, resulting in monopoly profits for the commer-

Park by Ossur, both producers of prosthetic devices (see OECD, 2020).

${ }^{6}$ See Bourreau and de Streel (2019), Crémer et al. (2019), Furman et al. (2019) and Cabral (2020).

${ }^{7} \mathrm{~A}$ prominent example for different approaches to an innovation is the development of the internet. Among several competing methods to connect different networks and transmit data, the packet switching method turned out to be the one efficient enough to enable today's internet (Leiner, Cerf, Clark, Kahn, Kleinrock, Lynch, Postel, Roberts and Wolff, 2009). 
cializing firm. Otherwise, it will be non-drastic, allowing the entrant to compete. Under a laissez-faire policy, the incumbent can acquire the entrant once the innovation outcomes become common knowledge. If an acquisition takes place, the trading surplus is split according to exogenously given shares reflecting bargaining power. ${ }^{8}$ The firm possessing the innovation technology then decides whether to commercialize it at some fixed cost or not; thereafter, product market competition takes place. Our model addresses the case when commercialization costs are high, so that the incumbent does not commercialize the acquired innovation (the killer acquisition case), as well as the case where they are low enough that it does (the genuine acquisition case). This is important because both cases are empirically relevant and because it might be difficult for the authorities to distinguish between them. ${ }^{9}$

We fully characterize the equilibrium structure, which enables us to analyze policy effects on innovation strategies. We first focus on the effects of prohibiting start-up acquisitions. The analysis is non-trivial because incumbents and entrants react differently to the policy. Nevertheless, we obtain the clear result that the policy effect on innovation is weakly negative. While this appears to vindicate the entry-for-buyout argument, there is an important qualification: The innovation effect is strictly negative in the killeracquisitions case, but in the genuine acquisitions case there is a non-degenerate parameter region where it is entirely absent. Thus, perhaps surprisingly, even though killer acquisitions may appear to be particularly problematic because non-drastic innovations do not reach the market, the pro-competitive argument for prohibiting genuine acquisitions is sometimes even clearer because the adverse innovation effect may be zero. Crucially, in all equilibria in the killer-acquisition case, the entrant's incentives determine the variety of innovation projects. As the absence of the acquisition option reduces the entrant's investment incentives, overall variety declines when acquisitions are prohibited. By contrast, when non-drastic innovations are valuable enough for the incumbent to commercialize, her incentives to innovate may be higher than those of the entrant. In this case, the incumbent's incentives are decisive for the variety of innovation, and they are not affected by the policy regime. Without an adverse innovation effect, prohibiting acquisitions improves welfare because it exclusively enhances competition.

In all other cases, however, policy has to trade off the positive competition effect of preventing acquisitions against the negative innovation effect. To this end, it is useful to understand for which market characteristics the innovation effect is small. Our results

\footnotetext{
${ }^{8}$ See Phillips and Zhdanov (2013), Cabral (2018) and Kamepalli, Rajan and Zingales (2020) for similar assumptions.

${ }^{9}$ This distinction mirrors the contrast between killer acquisitions and nascent potential competitor theory of harms. The latter case arises if "the acquired product might grow into a rival product, and hence ... controlling that product (but not killing it), removes the competitive threat that it poses" (OECD, 2020, p.7).
} 
suggest that the pro-competitive effect of prohibiting acquisitions is likely to dominate the adverse innovation effect in markets in which the entrant's bargaining power is low and the incumbent's competitive profits are high. Thus, innovation effects should not be seen as a carte blanche for allowing acquisitions. Rather, whether or not acquisitions should be allowed depends on the specifics of the industry.

Determining whether the market conditions justify an intervention may be difficult in practice. In Section 6, we therefore consider several alternative policies. First, we discuss two behavioral remedies: restrictions on the use of the acquired technology and prohibition of "killing" the acquired technology. Such remedies may decrease ex-ante innovation incentives, but in complementary cases: Limiting the usage of the acquired technology after an acquisition does not affect innovation for killer acquisitions, but decreases innovation for genuine acquisitions and may turn some of them into killer acquisitions. If the "killing" of the entrant's technology is prohibited, some killer acquisitions become genuine. Innovation is unaffected in the genuine-acquisition case and diminished in the killer-acquisition case. Second, we analyze tax policies which aim to tilt the decision of the start-up in favor of market entry. Similarly to the behavioral remedies, an increase in acquisition taxes is likely to decrease innovation. In contrast, making IPOs more profitable for startups, for instance by lowering the tax burden, fosters innovation. A common attractive feature of all these policy instruments is that, if they have an effect, they may render the acquisition unprofitable in circumstances when an entrant would make substantial profits on its own, suggesting that he would be a viable competitor.

We chose our modeling assumptions so as to avoid non-interesting cases. First, we assume that monopoly profits are higher than total duopoly profits, which makes an acquisition attractive. Second, we assume that entrants can, in principle, commercialize the innovation themselves, as otherwise prohibiting acquisitions would not have any competitive benefits.

Further, we show in Section 7 that the weakly negative innovation effects of a noacquisition policy are robust to relaxing other assumptions of the benchmark model. First, suppose that the size of the innovation (drastic or non-drastic) is not yet known at the time of the acquisition. Such uncertainty leads to more acquisitions, but has no effect on innovation behavior with or without a no-acquisition policy. Second, asymmetries in commercialization costs and the chances of receiving the patent do not influence the innovation effect, though, in the former case, a no-acquisition policy may suffer from the additional inefficiency that an entrant with high commercialization costs ends up commercializing the innovation. Third, when projects are heterogeneous (so that more costly projects are more likely to generate drastic innovations), banning acquisitions may have a particularly pronounced negative effect on the probability of drastic innovations. Fourth, we argue that, while the existence of a second entrant would tend to reduce investment incentives, 
the effect of the no-acquisition policy would remain qualitatively similar.

Finally, the reader may wonder how our predictions compare with those obtained in a more standard model where firms only choose the overall R\&D effort. ${ }^{10}$ In Section B.7, we analyze such a model and show that prohibiting acquisitions reduces the entrant's R\&D investment, but increases the incumbent's investment - because R\&D investment is now the incumbent's only way to block the entrant. For suitable parameters, the positive effect on the incumbent can dominate the negative effect on the entrant, resulting in a higher overall innovation probability. However, as our results reveal, this does not hold when firms can choose in which $R \& D$ projects to invest. The reason is that the incumbent, who seeks to block the entrant, only increases investments in those projects in which the entrant also invests (i.e., duplicate projects) and does not increase investments in new projects. Due to this increase in R\&D duplication, the prohibition of start-up acquisitions may increase the overall $R \& D$ investment, while nevertheless resulting in a lower probability of discovering the innovation. Compared to the standard framework where firms only choose the R\&D intensity, our framework identifies a novel effect of a more restrictive acquisition policy (change in duplication) and offers qualitatively different predictions (no positive innovation effect). Of course, which of the two modeling frameworks is adequate depends on the innovation technology for the case at hand, with the multi-project setting being more appropriate whenever there is fundamental uncertainty about the right approach.

The remainder of the paper is organized as follows. In the main text, we focus on the introduction of the main framework, the results and the discussion. Appendix A contains the statement of a result that is fundamental for the proofs of our equilibrium characterizations. Appendix B provides the details of the proofs as well as precise statement of results that we only mention briefly in the main text.

\section{Relation to the Literature}

Cunningham et al. (2021) not only provide empirical evidence for the existence of killer acquisitions, but they also develop a theoretical model to explain the rationale behind discontinuing development. The main difference between their model and ours is that we emphasize the initial innovation decisions, which they do not analyze.

A recent theoretical literature has studied under which circumstances mergers of incumbents increase innovation. Federico, Langus and Valletti $(2017,2018)$ and Motta and Tarantino (2018) identify negative effects, whereas Denicolò and Polo (2018) find positive effects. In Bourreau, Jullien and Lefouili (2019), both possibilities arise. ${ }^{11}$ In models with

\footnotetext{
${ }^{10}$ Such models are in the tradition of, for example, Gilbert and Newbery (1982), Reinganum (1983), Loury (1979) and Lee and Wilde (1980).

${ }^{11}$ A related literature investigates the effects of the number of firms on innovation, see Yi (1999), Norbäck and Persson (2012) and Marshall and Parra (2019). Moreover, many papers discuss the relation
} 
multiple research approaches, Letina (2016) and Gilbert (2019) obtain negative effects on $R \& D$ diversity; Letina also finds that mergers reduce research duplication. MoragaGonzález, Motchenkova and Nevrekar (2019) show that mergers may increase welfare by alleviating biases in the direction of innovation. ${ }^{12}$

Instead of focusing on incumbent innovations, our paper asks how acquisition policy affects the R\&D project choices of incumbents and entrants. ${ }^{13}$ Rasmusen (1988) identified an incentive to enter a market to get bought by the current incumbent, suggesting that a lenient acquisition policy can foster innovative entry. ${ }^{14}$ In Phillips and Zhdanov (2013) a laissez-faire policy increases the incumbent's innovation as well as the entrant's. ${ }^{15}$ Cabral (2018) obtains the innovation-for-buyout effect in a continuous-time setting. Mermelstein, Nocke, Satterthwaite and Whinston (2020) and Hollenbeck (2020) use computational methods to study the long-run effects of merger policy in dynamic oligopoly models with entry-for-buyout incentives; the latter finds that prohibiting mergers can lead to less innovation and lower long-run consumer welfare. Fumagalli, Motta and Tarantino (2020) show that, in spite of potential anti-competitive effects, the prospect of acquisitions by an incumbent may foster start-up innovations by relaxing financial constraints. ${ }^{16}$ By contrast, Kamepalli et al. (2020) and Katz (2020) argue that, in the tech industry, a laissez-faire policy may have negative effects on start-up innovations. ${ }^{17}$ While Gans and Stern (2000) focus less on acquisition policies, they provide an in-depth analysis of the innovation decisions of entrants who can bargain with incumbents about cooperative agreements such as technology licensing. They show how the terms of the agreement depend on fundamentals such as property rights and stand-alone profits. ${ }^{18}$ Unlike our paper, none of these papers analyzes the strategic choice of innovation projects.

Several papers deal with the effects of acquisition policy on the type of innovation. Bryan and Hovenkamp (2020a) consider distortions in the innovation decisions of start-ups

between other measures of competitive intensity and innovation; see Vives (2008) and Schmutzler (2013) for unifying approaches.

${ }^{12}$ Bryan and Lemus (2017), Letina and Schmutzler (2019), Bardey, Jullien and Lozachmeur (2016) and Bavly, Heller and Schreiber (2020) treat other aspects of innovation project choice.

${ }^{13}$ Segal and Whinston (2007) ask how the antitrust treatment of incumbents affects entrants' innovation incentives: A more restrictive policy increases the entrants' short-term benefits from being in the market, but leads to long-term losses in case he becomes dominant himself.

${ }^{14}$ See Mason and Weeds (2013) for similar reasoning.

${ }^{15}$ In their model, large firms can sell their own product and the target's product after the acquisition and there is an additional value from applying an innovation to both products.

${ }^{16}$ The related contribution of Motta and Peitz (2021) focuses mainly on the ex-post acquisition and commercialization decisions of a resource-constrained entrant who has previously generated an innovation.

${ }^{17}$ While the results of the two papers are similar, the central mechanisms differ. In Kamepalli et al. (2020), expectations of "techies" (potential early adopters of a new technology) drive the result. In Katz (2020), the key assumption is that potential entrants can choose innovation quality.

${ }^{18}$ They use these insights to investigate the effects of the acquisition option on innovation incentives and on the nature of the strategic interaction between firms. Gans, Hsu and Stern (2002) provides empirical support for the predictions. 
who produce inputs for competing incumbents, without considering entry into this competition. In Gilbert and Katz (2021) and Dijk, Moraga-Gonzalez and Motchenkova (2021), a vertically differentiated entrant can choose whether or not to compete head-to-head with the incumbent. The papers provide conditions under which a restrictive acquisition policy will increase or decrease biases in this decision. In Callander and Matouschek (2021), the entrant can similarly choose the distance to the incumbent's location. The prospect of an acquisition incentivizes the entrant to locate closer to the existing product, and hence to aim for a less radical and less uncertain innovation. ${ }^{19}$

Contrary to these papers with a new take on the issue of product differentiation, we emphasize differentiation in the innovation process, in the projects that firms apply to achieve a given innovation goal. More generally, compared with the existing literature, we focus on identifying market characteristics driving the size of the innovation effect and justifying intervention. On a related note, we show how the case for intervention differs between killer acquisitions and genuine acquisitions. Our emphasis on innovation portfolios allows us to analyze policy effects on project variety and duplication rather than merely on overall innovation efforts. Finally, to our knowledge, we are the first to provide a formal analysis of the innovation effects of a wide range of policies towards start-up acquisitions.

\section{The Model}

We consider two variants of a multi-stage game, corresponding to a laissez-faire policy (A) which tolerates acquisitions and a no-acquisition policy (N). In both cases, there is an incumbent $(i=I)$ who owns a technology with which she can produce goods. In addition, she can invest in $\mathrm{R} \& \mathrm{D}$. An entrant $(i=E)$ has to invest in $\mathrm{R} \& \mathrm{D}$ before he can produce. Before providing the details, we start with an overview of the time structure.

1. Investment stage: Firms simultaneously decide how much to invest in different research projects, thereby determining the probability of a (patentable) innovation, which can be drastic or non-drastic with exogeneously given probability.

2. Acquisition stage: Under a laissez-faire policy firms negotiate an acquisition, which takes place if and only if it strictly increases total payoffs, and they negotiate the acquisition price. Under a no-acquisition policy, this stage is dropped.

\footnotetext{
${ }^{19}$ Similar to Callander (2011) and Carnehl and Schneider (2021), the authors postulate a positive relation between distance and novelty of an innovation. Cabral (2018) derives a similar conclusion to Callander and Matouschek (2021) in a very different setting. In Wickelgren (2021), lenient acquisition policy encourages entrants to develop substitutes rather than complements to the incumbent's product. Motta, Shelegia et al. (2021) identify a tendency for rivals to provide complements rather than substitutes to an incumbent's products to stay out of the kill zone (avoid being copied), but argue that the prospect of acquisitions works against this effect, pushing entrants towards developing substitutes.
} 
3. Commercialization stage: The firm holding the patent (if any) decides whether to commercialize the technology.

4. Market stage: The incumbent and the entrant receive product market profits, which depend on whether there was an innovation, whether it was drastic or nondrastic and which firm has access to it. Total payoffs result after accounting for potential investment and commercialization costs and acquisition payments.

We now describe the stages in detail. In the investment stage, the firms choose how much to invest into each research project $\theta$ from a continuum $\Theta=[0,1)$. Only one project, $\hat{\theta} \in \Theta$, will result in an innovation (be the correct project). All other projects will lead to a dead end and produce no valuable output. Each project is equally likely to be correct. For all $\theta \in[0,1)$, each firm chooses a research intensity $r_{i}(\theta) \in[0,1] . r_{i}(\hat{\theta})$ is the probability that firm $i$ will discover the innovation if $\hat{\theta}$ is the correct project. We restrict the firms' choices to the set $\mathcal{R}$ of measurable functions $r:[0,1) \rightarrow[0,1]$. The investment cost of firm $i$ is $\int_{0}^{1} r_{i}(\theta) C(\theta) d \theta$, where the cost function $C:[0,1) \rightarrow \mathbb{R}_{+}$is continuous, differentiable, strictly increasing and convex and such that $\lim _{\theta \rightarrow 1} C(\theta)=\infty$ and $C(0)=0$.

With exogeneously given probability $p$, the correct project $\hat{\theta}$ results in a high technological state $(H)$, corresponding to a drastic innovation compared to the incumbent's current technology. ${ }^{20}$ Otherwise, $\hat{\theta}$ results in a low state $L$, corresponding to a non-drastic innovation, allowing the entrant to compete with the incumbent and obtain positive market profits. If a single firm discovers the innovation, it receives a patent. If both firms discover the innovation, the patent is allocated randomly with equal probability. ${ }^{21}$ We assume that only the patent holder can use the new technology. Once the correct project has been realized, both firms learn the resulting technology level, summarized in the interim technology states $\left(t_{I}^{i n t}, t_{E}^{i n t}\right) \in \mathcal{T}:=\{(\ell, 0),(\ell, L),(\ell, H),(L, 0),(H, 0)\}$, where $\ell$ corresponds to the incumbent's initial technology and 0 corresponds to the entrant's initial technology. ${ }^{22}$

In the second stage of the game under laissez-faire, the acquisition stage, the incumbent can acquire the entrant by paying the profits that the latter could obtain in the market plus a share of the (bargaining) surplus $\beta \in(0,1)$. We will assume that the acquisition takes place if and only if the bargaining surplus is strictly positive. If the entrant is acquired, then any patent held by the entrant is transferred to the incumbent. In the third stage, the commercialization stage, the patent holder can bring the new technology to the market at commercialization cost $\kappa>0 .{ }^{23}$ Thereafter, the final technology states $\left(t_{I}^{\text {fin }}, t_{E}^{\text {fin }}\right) \in \mathcal{T}$

\footnotetext{
${ }^{20}$ This is a variant of the standard assumption that the size of the profit effect of an innovation is not perfectly predictable given the R\&D investment of a firm. In Section 7, we find that, when more costly projects are more likely to generate drastic innovations, similar results emerge.

${ }^{21}$ We consider asymmetric chances of receiving patents in Section 7.

${ }^{22}$ More technically, we assume that, simultaneously with the innovation decisions, there is a move of nature determining the correct project and whether the innovation is drastic or non-drastic.

${ }^{23}$ As we show in Section 7 , none of our main insights depend on $\kappa$ being equal for both firms.
} 
result. Finally, in the product market stage, each firm $i \in\{I, E\}$ with technology $t_{i}$ facing a competitor $j$ with technology $t_{j}$, collects product market profits $\pi_{i}\left(t_{i}, t_{j}\right)$. We introduce the following assumptions.

Assumption 1 (Market profits).

(i) Profits are non-negative: $\pi_{i}\left(t_{i}, t_{j}\right) \geq 0$ for any $t_{i}$ and $t_{j}$. Monopoly profits are strictly positive, that is, $\pi_{i}\left(t_{i}, 0\right)>0$ for any $t_{i}$.

(ii) Without an innovation, the entrant cannot compete: $\pi_{E}\left(0, t_{I}\right)=0$ for $t_{I} \in\{\ell, L, H\}$.

(iii) Technology $H$ corresponds to a drastic innovation and generates monopoly profit: $\pi(H):=\pi_{E}(H, \ell)=\pi_{I}(H, 0)>\max \left\{\pi_{I}(L, 0), \pi_{I}(\ell, 0)\right\}$ and $\pi_{I}(\ell, H)=0$.

(iv) Competition decreases total profits:

$\max \left\{\pi_{I}(L, 0), \pi_{I}(\ell, 0)\right\}>\pi_{I}(\ell, L)+\pi_{E}(L, \ell)$.

Assumption 1(ii) captures the fundamental asymmetry between incumbent and entrant. We allow profits to be firm-specific functions of technological states, except for drastic innovations, see Assumption 1(iii). Finally, Assumption 1(iv) ensures that the incumbent wishes to acquire the entrant at least sometimes. Assumption 1 is consistent with a wide range of interpretations, applying equally to process and product innovations. In the latter case, we do not rule out that an incumbent will produce her old product as well as the entrant's: One can simply interpret $\pi_{I}(L, 0)$ as corresponding to a multiproduct monopoly profit. Assumption 1(iv) is natural in this case as well, because a two-product monopolist can always imitate the pricing of differentiated duopolists and thus earn at least as much.

Assumption 2. Commercialization costs satisfy

$$
\begin{aligned}
& \text { (i) } \pi_{E}(L, \ell) \geq \kappa ; \\
& \text { (ii) } \pi(H)-\pi_{I}(\ell, 0) \geq \kappa .
\end{aligned}
$$

Thus, even with the non-drastic innovation, the entrant's profit is at least as high as the commercialization cost. This avoids the case that the entrant is not viable on its own, in which prohibiting acquisitions would not have any pro-competitive effect. For the incumbent, the increase in the monopoly profit obtained by using the drastic innovation outweighs the commercialization cost. For the non-drastic innovation, this may or may not be the case.

We refer to the firms' continuation payoffs at the beginning of the acquisition stage, conditional on the realization of the interim states $t_{I}^{\text {int }}$ and $t_{E}^{\text {int }}$, as their values $v_{I}\left(t_{I}^{\text {int }}, t_{E}^{\text {int }}\right)$ and $v_{E}\left(t_{E}^{i n t}, t_{I}^{i n t}\right)$, respectively. These values depend on the policy regime. They are independent of the competitor state if a firm's state is $H$; we thus simply write $v_{I}(H)$ and 
$v_{E}(H)$. The expected total payoff of the incumbent who chooses an investment function $r_{I}(\theta)$ when facing an entrant who chooses $r_{E}(\theta)$ is

$$
\begin{aligned}
\mathbb{E} \Pi_{I}\left(r_{I}, r_{E}\right)= & -\int_{0}^{1} r_{I}(\theta) C(\theta) d \theta+\int_{0}^{1} r_{I}(\theta)\left(1-r_{E}(\theta)\right)\left[p v_{I}(H)+(1-p) v_{I}(L, 0)\right] d \theta \\
& +\int_{0}^{1}\left(1-r_{I}(\theta)\right) r_{E}(\theta)(1-p) v_{I}(\ell, L) d \theta+\int_{0}^{1}\left(1-r_{I}(\theta)\right)\left(1-r_{E}(\theta)\right) v_{I}(\ell, 0) d \theta \\
& +\int_{0}^{1} r_{I}(\theta) r_{E}(\theta)\left[p\left(\frac{1}{2} v_{I}(H)\right)+(1-p)\left(\frac{1}{2} v_{I}(L, 0)+\frac{1}{2} v_{I}(\ell, L)\right)\right] d \theta .
\end{aligned}
$$

The first integral captures the innovation costs of an incumbent with strategy $r_{I}$. The second integral represents the incumbent's continuation payoff when she discovers an innovation and the entrant does not, conversely for the third integral. The fourth integral represents the continuation payoff when neither firm innovates, and the fifth is for the case when both firms innovate. Similarly, for the entrant we obtain:

$$
\begin{aligned}
\mathbb{E} \Pi_{E}\left(r_{E}, r_{I}\right)= & -\int_{0}^{1} r_{E}(\theta) C(\theta) d \theta+\int_{0}^{1} r_{E}(\theta)\left(1-r_{I}(\theta)\right)\left[p v_{E}(H)+(1-p) v_{E}(L, \ell)\right] d \theta \\
& +\int_{0}^{1} r_{E}(\theta) r_{I}(\theta)\left[\frac{p}{2} v_{E}(H)+\frac{1-p}{2} v_{E}(L, \ell)\right] d \theta .
\end{aligned}
$$

For the investment stage, characterizing subgame-perfect equilibria amounts to finding functions $r_{i}, r_{j} \in \mathcal{R}$ such that $\mathbb{E} \Pi_{i}\left(r_{i}, r_{j}\right) \geq \mathbb{E} \Pi_{i}\left(r_{i}^{\prime}, r_{j}\right)$ for any $r_{i}^{\prime} \in \mathcal{R} .{ }^{24}$ However, because of the additively separable structure of the objective functions, the game can effectively be decomposed into a continuum of investment games, one for each project. Thus, for any project $\theta$, to find the best-reply investment of firm $i$ we only need to look at the amount invested by the other firm $r_{j}(\theta)$ and we can ignore the investments of both firms in all other projects, which simplifies the equilibrium analysis significantly. Using this approach, we will show that the characterization of the equilibrium investment will rely on critical projects $\theta_{E}^{1}, \theta_{E}^{2}, \theta_{I}^{1}$ and $\theta_{I}^{2}$, which are defined implicitly by:

$$
\begin{aligned}
C\left(\theta_{E}^{1}\right) & =p v_{E}(H)+(1-p) v_{E}(L, \ell) \\
C\left(\theta_{E}^{2}\right) & =\frac{1}{2}\left(p v_{E}(H)+(1-p) v_{E}(L, \ell)\right) \\
C\left(\theta_{I}^{1}\right) & =p v_{I}(H)+(1-p) v_{I}(L, 0)-v_{I}(\ell, 0) \\
C\left(\theta_{I}^{2}\right) & =\frac{p}{2} v_{I}(H)+(1-p)\left(\frac{1}{2} v_{I}(L, 0)+\frac{1}{2} v_{I}(\ell, L)\right)-(1-p) v_{I}(\ell, L) .
\end{aligned}
$$

The expected cost of a critical project equals the expected future profit increase it

\footnotetext{
${ }^{24}$ Obviously, for any equilibrium $\left(r_{I}, r_{E}\right)$, any pair of functions $\left(\tilde{r}_{I}, \tilde{r}_{E}\right)$ which only differ from $\left(r_{I}, r_{E}\right)$ on a set of measure zero is also an equilibrium. We omit the necessary "almost everywhere" qualifications from the statements of our formal results for ease of exposition.
} 
generates. The critical projects differ for incumbents and entrants and depend on whether the competitor is expected to invest in the same project or not. Accordingly, project $\theta_{i}^{1}$ is defined by the requirement that its cost equals the expected value increase to firm $i$ if it invests in the correct project when the other firm does not. Since project costs are increasing in $\theta$, this implies that firm $i$ would want to invest in any $\theta \in\left[0, \theta_{i}^{1}\right)$ for which it assumes that the competitor does not invest in, and it would not want to invest in any $\theta \in\left(\theta_{i}^{1}, 1\right)$ in which it believes the competitor is not investing. Similarly, $\theta_{i}^{2}$ is defined by the requirement that its cost equals the expected value increase to firm $i$ if it invests in a correct project in which the other firm invests as well.

\section{Investments under the Laissez-Faire Policy}

We now analyze investments in the laissez-faire case. In Section 4.1, we provide some auxiliary results. In Section 4.2, we characterize equilibrium investments.

\subsection{Auxilliary Results}

We begin by summarizing the result of the acquisition subgame.

Lemma 1 (Acquisitions). Under laissez-faire, the incumbent acquires the entrant if and only if the latter holds a patent for technology L. Commercialization arises in any commercialization subgame, except if the incumbent holds the patent and $\pi_{I}(L, 0)-\pi_{I}(\ell, 0)<\kappa$.

Intuitively, if the entrant owns technology $L$, an acquisition increases total profits by eliminating competition, but it leaves profits unaffected otherwise. The incumbent's commercialization decision depends on the value of the non-drastic innovation. If $\pi_{I}(L, 0)-$ $\pi_{I}(\ell, 0)<\kappa$ (henceforth, the killer-acquisition case), commercialization is not worthwhile

- the only motive for an acquisition is the elimination of competition. If $\pi_{I}(L, 0)-$ $\pi_{I}(\ell, 0) \geq \kappa$ (the genuine-acquisition case), the incumbent additionally benefits from a better technology.

Using Lemma 1, we obtain firm values after the realization of innovation outcomes.

Lemma 2 (Values). Consider the laissez-faire policy:

(i) The entrant's values after realization of the innovation outcomes are

$$
\begin{aligned}
& v_{E}(H)=\pi(H)-\kappa \\
& v_{E}(L, \ell)=\pi_{E}(L, \ell)-\kappa+\beta\left(\max \left\{\pi_{I}(L, 0)-\kappa, \pi_{I}(\ell, 0)\right\}-\pi_{E}(L, \ell)-\pi_{I}(\ell, L)+\kappa\right) \\
& v_{E}\left(0, t_{I}\right)=0 \text { for } t_{I} \in\{\ell, L, H\} .
\end{aligned}
$$

(ii) The incumbent's values after realization of the innovation outcomes are

$$
\begin{aligned}
& v_{I}(H)=\pi(H)-\kappa \\
& v_{I}(L, 0)=\max \left\{\pi_{I}(L, 0)-\kappa, \pi_{I}(\ell, 0)\right\}
\end{aligned}
$$




$$
\begin{aligned}
& v_{I}(\ell, L)=v_{I}(L, 0)-v_{E}(L, \ell) \\
& v_{I}(\ell, 0)=\pi_{I}(\ell, 0) \\
& v_{I}(\ell, H)=0 .
\end{aligned}
$$

The values involving technology $L$ require an explanation. After a non-drastic entrant innovation, $\left(t_{I}^{i n t}, t_{E}^{i n t}\right)=(\ell, L)$. The incumbent then acquires the entrant, so that $v_{E}(L, \ell)$ is the acquisition price (the sum of the entrant's stand-alone profit and his share of the surplus). $v_{I}(\ell, L)$ is the monopolist's stand-alone payoff, net of the acquisition price. Finally, the max-operators take into account the difference between the killer-acquisition and genuine-acquisition case. Using Lemma 2, we can now restrict the ordering of the critical projects, which is essential for the equilibrium properties.

Lemma 3. Under laissez-faire, the critical projects must satisfy (i), (ii) or (iii):

(i) $\theta_{I}^{1} \leq \theta_{I}^{2}=\theta_{E}^{2}<\theta_{E}^{1}$;

(ii) $\theta_{I}^{2}=\theta_{E}^{2}<\theta_{I}^{1}<\theta_{E}^{1}$;

(iii) $\theta_{I}^{2}=\theta_{E}^{2}<\theta_{E}^{1} \leq \theta_{I}^{1}$.

Relation (iii) cannot arise in the killer-acquisition case.

Lemma 3 reveals some common properties of all equilibria. First, the projects which the incumbent is willing to duplicate (i.e., invest in if the entrant also does) are exactly those which the entrant is willing to duplicate as well; we thus write $\theta^{2}:=\theta_{I}^{2}=\theta_{E}^{2} \cdot{ }^{25}$ Second, $\theta_{E}^{2}<\theta_{E}^{1}$, so that the entrant is always willing to invest in a larger range of projects if he is the sole innovator than if the incumbent also invests in these projects. Intuitively, the incumbent's investment reduces the entrant's probability of receiving a patent.

There is a crucial difference between the genuine- and killer-acquisition cases. While all three orderings can arise in the former case, $\theta_{I}^{1}<\theta_{E}^{1}$ holds in the killer-acquisition case, so that case (iii) is impossible. Intuitively, conditional on the other firm not investing, the entrant is willing to invest in more expensive projects than the incumbent. This reflects the well-known Arrow replacement effect: An $L$ innovation does not increase incumbent profits, and her profit increase from the $H$ innovation is lower than the entrant's, since without the innovation the entrant receives zero profits. Hence, the entrant's willingness to pay to be the sole innovator is greater than the incumbent's. This will be important for

\footnotetext{
${ }^{25}$ To understand why, note that if a project in which both firms invest delivers an $H$ technology, both firms receive the same expected net payoff from investing, because not investing means losing the high innovation to the rival and receiving 0 for sure rather than obtaining the high monopoly profit with probability $1 / 2$. If a project delivers an $L$ technology instead, the entrant gains the acquisition price with probability $1 / 2$ by investing, while the incumbent saves the acquisition price with probability $1 / 2$ by investing. Thus, the expected benefits of investing (conditional on the other firm investing) are the same for entrants and incumbents.
} 
our result that prohibiting acquisitions has a negative effect on equilibrium investments. Contrary to the killer-acquisition case, the incumbent's critical project $\theta_{I}^{1}$ may lie above the entrant's critical project $\theta_{E}^{1}$ in the genuine-acquisition case, as in ordering (iii). We will identify the circumstances under which this occurs and discuss the implications of this observation after Proposition 2 below.

\subsection{Equilibrium Investments}

We now characterize the equilibrium R\&D investments, using the following definition:

Definition 1. The firms use an anti-coordination profile on a subset $S$ of $[0,1)$ if, for every $\theta \in S$ one of the following cases arises:

(i) $r_{E}(\theta)=1$ and $r_{I}(\theta)=0$, or (ii) $r_{E}(\theta)=0$ and $r_{I}(\theta)=1$, or

(iii) $r_{E}(\theta)=\frac{C\left(\theta_{I}^{1}\right)-C(\theta)}{C\left(\theta_{I}^{1}\right)-C\left(\theta_{I}^{2}\right)}$ and $r_{I}(\theta)=\frac{C\left(\theta_{E}^{1}\right)-C(\theta)}{C\left(\theta_{E}^{1}\right)-C\left(\theta_{E}^{2}\right)} \cdot 26$

Thus, firms use an anti-coordination profile on $S$ if, for each project in $S$, either only one of the firms invests fully, whereas the other one does not invest at all, or both firms invest just enough to make the other firm indifferent between investing and not investing.

Our characterization result shows that both firms invest in all sufficiently cheap projects, but neither firm invests in the most expensive projects. Moreover, firms may choose an anti-coordination profile on some subset of projects.

Proposition 1 (Equilibrium R\&D investment). In any equilibrium under laissez-faire,

$$
\begin{aligned}
& \text { (A) } r_{E}(\theta)=1 \text { and } r_{I}(\theta)=1 \text { for } \theta \in\left[0, \theta^{2}\right], \\
& \text { (B) } r_{E}(\theta)=0 \text { and } r_{I}(\theta)=0 \text { for } \theta \in\left(\max \left\{\theta_{E}^{1}, \theta_{I}^{1}\right\}, 1\right) .
\end{aligned}
$$

(i) If $\theta_{I}^{1} \leq \theta^{2}<\theta_{E}^{1}$, then there exists a unique equilibrium. In addition to $(A)$ and $(B)$, this equilibrium satisfies $r_{E}(\theta)=1$ and $r_{I}(\theta)=0$ for $\theta \in\left(\theta^{2}, \theta_{E}^{1}\right]$.

(ii) If $\theta^{2}<\theta_{I}^{1}<\theta_{E}^{1}$, the equilibrium is not unique. A strategy profile is an equilibrium if and only if it satisfies $(A)$ and $(B)$, firms use an anti-coordination profile on $\left(\theta^{2}, \theta_{I}^{1}\right]$, and $r_{E}(\theta)=1$ and $r_{I}(\theta)=0$ for $\theta \in\left(\theta_{I}^{1}, \theta_{E}^{1}\right]$.

(iii) If $\theta^{2}<\theta_{E}^{1} \leq \theta_{I}^{1}$, the equilibrium is not unique. A strategy profile is an equilibrium if and only if it satisfies $(A)$ and $(B)$, firms use an anti-coordination profile on $\left(\theta^{2}, \theta_{E}^{1}\right]$, and $r_{E}(\theta)=0$ and $r_{I}(\theta)=1$ for $\theta \in\left(\theta_{E}^{1}, \theta_{I}^{1}\right]$.

\footnotetext{
${ }^{26}$ Note that we are not requiring the same case to apply for every $\theta \in S$.
} 
We will refer to equilibria with $r_{E}(\theta) \in\{0,1\}$ and $r_{I}(\theta) \in\{0,1\} \forall \theta \in[0,1)$ as simple equilibria. Proposition 1 implies that, under laissez-faire, a simple equilibrium exists for any choice of parameters, in the killer- as well as the genuine-acquisition case. In case $(i)$, depicted in the left plot of Figure 1, both firms invest fully $\left(r_{i}=1\right)$ in all projects in the interval $\left[0, \theta^{2}\right]$, while only the entrant invests in $\left(\theta^{2}, \theta_{E}^{1}\right]$. Neither firm invests in projects in $\left(\theta_{E}^{1}, 1\right)$. In case $(i i)$, this simple equilibrium coexists with infinitely many other (simple and non-simple) equilibria, because in any project in $\left[\theta^{2}, \theta_{I}^{1}\right)$ each firm only wants to invest if the other one does not, so that firms use an anti-coordination profile. The middle plot of Figure 1 shows an equilibrium where both choose intermediate investment levels in $\left(\theta^{2}, \theta_{I}^{1}\right]$.
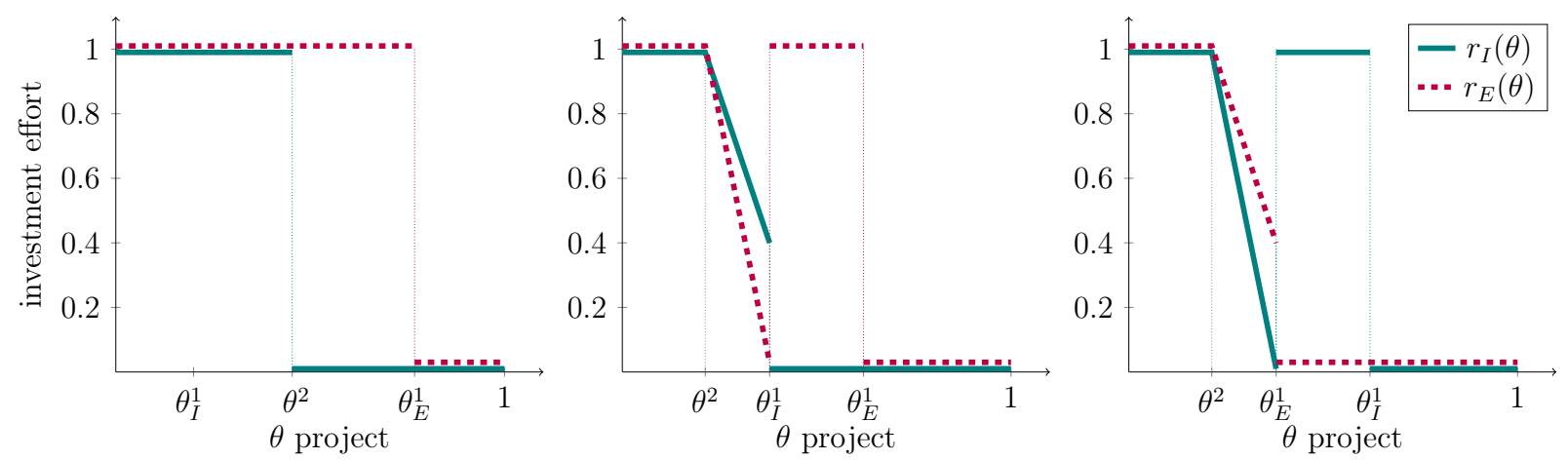

Figure 1: Equilibrium portfolio of entrant and incumbent for the three cases of Proposition 1: Case (i) in the left, case (ii) in the middle and case (iii) in the right plot.

In the killer-acquisition case, only the equilibrium constellations (i) and (ii) can arise. For genuine acquisitions, the incumbent has additional investment incentives, as the commercialization of non-drastic innovations may increase monopoly profits. Nevertheless, if $\theta_{I}^{1}<\theta_{E}^{1}$ as with killer acquisitions, the equilibrium structure is the same, and the entrant's critical project $\theta_{E}^{1}$ is the most costly one pursued in equilibrium. The possibility that $\theta_{I}^{1}$ lies above $\theta_{E}^{1}$ (case iii) has repercussions for the equilibrium structure. In this case, the multiple equilibrium structure of Case (ii) is replaced by one where the incumbent and the entrant switch roles - otherwise it is entirely analogous. The right plot of Figure 1, which corresponds to Proposition 1(iii), shows one potential equilibrium when $\theta_{I}^{1}>\theta_{E}^{1}$. As depicted, in all equilibria in this last case, the incumbent's critical project is the most costly one pursued.

Proposition 1 implies that in any equilibrium there exists a set of projects in which only the firm with higher $\theta_{i}^{1}$ invests. In the killer-acquisition case (where (i) or (ii) applies), decreasing the entrant's innovation incentives will cause him to reduce investment in exactly the projects which cost the most and which only he would pursue. When the acquisition is genuine, this logic no longer applies in case (iii), as the incumbent now is the one whose critical project $\theta_{I}^{1}$ is the most costly one pursued. 
Given any strategy profile $\left(r_{I}, r_{E}\right)$, the probability that at least one firm innovates is

$$
\mathcal{P}\left(r_{I}, r_{E}\right)=\int_{0}^{1}\left(r_{I}(\theta)+r_{E}(\theta)-r_{I}(\theta) r_{E}(\theta)\right) d \theta .
$$

However, the innovation probability is sensitive to equilibrium selection. As an alternative, we therefore introduce the variety of research projects which is equal to the size of the set of projects in which at least one firm invests. Formally:

$$
\mathcal{V}\left(r_{I}, r_{E}\right)=\int_{0}^{1} \mathbf{1}\left(r_{I}(\theta)+r_{E}(\theta)>0\right) d \theta .
$$

The following implication of Proposition 1 shows that variety is a useful proxy for probability: It is invariant to equilibrium selection, provides an upper bound to the innovation probability in any equilibrium and coincides with it in any simple equilibrium.

Corollary 1. Under a laissez-faire policy, if $\left(r_{I}, r_{E}\right)$ is an equilibrium, then $\mathcal{V}\left(r_{I}, r_{E}\right)=$ $\max \left\{\theta_{E}^{1}, \theta_{I}^{1}\right\} \geq \mathcal{P}\left(r_{I}, r_{E}\right)$. If $\left(r_{I}, r_{E}\right)$ is a simple equilibrium, then $\mathcal{V}\left(r_{I}, r_{E}\right)=\mathcal{P}\left(r_{I}, r_{E}\right)$.

There are two reasons why we consider non-simple equilibria in spite of this attractive feature of simple equilibria. First, it may be easier to coordinate on non-simple equilibria. Second, while simple equilibria always exist under laissez-faire, when acquisitions are prohibited, simple equilibria do not always exist (see Section B.2.2).

\section{Prohibiting Acquisitions}

We now analyze the effects of prohibiting start-up acquisitions. In Section 5.1, we show that this policy reduces the equilibrium project variety and innovation probability. Section 5.2 analyzes how the size of this effect depends on the market environment. In Section 5.3, we discuss the effect of the policy on $R \& D$ duplication. We denote the critical values under the laissez-faire and no-acquisition policies as $\theta_{i}^{k}(A)$ and $\theta_{i}^{k}(N), k \in\{1,2\}$, respectively.

\subsection{The Effect on Variety}

Firm behavior in the commercialization and market stages remains unchanged when acquisitions are prohibited. By Lemma 1, such a policy constrains behavior only when the entrant has a non-drastic innovation. Analogously to the laissez-faire case, in any equilibrium $\left(r_{I}^{N}, r_{E}^{N}\right)$ for the no-acquisition policy the firms invest in all projects below $\max \left\{\theta_{E}^{1}(N), \theta_{I}^{1}(N)\right\}$, but in no other projects. ${ }^{27}$ Hence, variety in this regime is given by $\mathcal{V}^{N}=\max \left\{\theta_{E}^{1}(N), \theta_{I}^{1}(N)\right\}$. Since by Corollary 1 variety in any laissez-faire equilibrium

\footnotetext{
${ }^{27}$ We provide a full characterization of the equilibria under the no-acquisition policy in Propositions B.1 and B.2 in Appendix B.2.2.
} 
is $\mathcal{V}^{A}=\max \left\{\theta_{E}^{1}(A), \theta_{I}^{1}(A)\right\}$, the size of the policy effect on variety is $\Delta_{\mathcal{V}}:=\mathcal{V}^{A}-\mathcal{V}^{N}=$ $\max \left\{\theta_{E}^{1}(A), \theta_{I}^{1}(A)\right\}-\max \left\{\theta_{E}^{1}(N), \theta_{I}^{1}(N)\right\}$. Our next result characterizes the sign of $\Delta_{\mathcal{V}}$.

Proposition 2. Consider the no-acquisition policy.

(i) In any equilibrium, (a) the variety of research projects is weakly smaller than in any equilibrium under laissez-faire and (b) the probability of an innovation is weakly smaller than in any simple equilibrium under laissez-faire.

(ii) The inequalities in (i) are strict, except that there is no effect on variety in the genuine-acquisition case if $\theta_{E}^{1}(A) \leq \theta_{I}^{1}(A)$.

Proposition 2 shows that a restrictive acquisition policy never increases variety. However, (ii) highlights a crucial difference between genuine and killer acquisitions. The policy effect is strictly negative in the latter case, but not necessarily in the former. This reflects two simple observations. First, $\theta_{E}^{1}(N)<\theta_{E}^{1}(A)$ : Prohibiting acquisitions reduces the entrant's expected payoff from R\&D investments, since he cannot sell the firm. Second, $\theta_{I}^{1}(A)=\theta_{I}^{1}(N)=: \theta_{I}^{1}$ : If the entrant does not invest in the correct project, there will be no reason to acquire him, so that the policy regime is irrelevant for $\theta_{I}^{1}$. Only three possible orderings for $\theta_{I}^{1}$ and the entrant's critical projects $\theta_{E}^{1}(A)$ and $\theta_{E}^{1}(N)$ are compatible with these two observations:

$$
\begin{array}{r}
\text { (I) } \theta_{I}^{1}<\theta_{E}^{1}(N)<\theta_{E}^{1}(A) \\
\text { (II) } \theta_{E}^{1}(N) \leq \theta_{I}^{1}<\theta_{E}^{1}(A) \\
\text { (III) } \theta_{E}^{1}(N)<\theta_{E}^{1}(A) \leq \theta_{I}^{1} .
\end{array}
$$

When (I) or (II) applies, $\theta_{E}^{1}(A)$, which reflects the entrant's incentives, determines the equilibrium variety under laissez-faire. A ban on acquisitions weakens these incentives and therefore reduces variety to $\theta_{E}^{1}(N)$ under ordering (I) or to $\theta_{I}^{1}$ under (II). Figure 2(I) and 2 (II) illustrate these two cases, respectively. When (III) applies, $\theta_{I}^{1}$ determines the equilibrium variety in both policy regimes. Hence, as illustrated in Figure 2(III), a prohibition of acquisitions has no effect. Importantly, ordering (III) only applies when acquisitions are genuine, which implies that the policy effect is strict for all killer acquisitions. Furthermore, since $\mathcal{P}\left(r_{I}, r_{E}\right)=\mathcal{V}\left(r_{I}, r_{E}\right)$ in any simple equilibrium, the statement in Proposition 2 on innovation probabilities immediately follows from the effect on variety.

Proposition 2(ii) gives a condition under which a prohibition of acquisitions has no innovation effect at all, coinciding with case (iii) in Lemma 3 (or case (III) in Figure 2). A comparison of the definition of $C\left(\theta_{I}^{1}\right)$ and $C\left(\theta_{E}^{1}\right)$ shows that this happens if and only if $(1-p) v_{I}(L, 0)-v_{I}(\ell, 0) \geq(1-p) v_{E}(L, \ell)$. Proposition B.3 in Appendix B.3.2 expresses this condition in terms of fundamentals. We find that a necessary condition for 


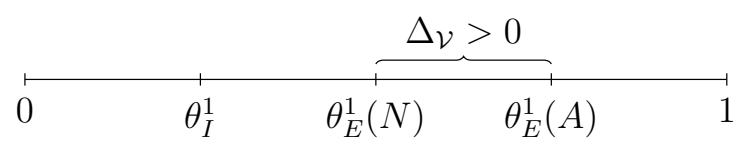

$$
\stackrel{\theta_{E}^{1}(N) \quad \theta_{I}^{1} \quad \theta_{E}^{1}(A)}{\theta_{\mathcal{V}}>0}
$$

$$
\begin{aligned}
& \Delta_{\mathcal{V}}=0 \\
& \begin{array}{lllll}
\longmapsto & \theta_{E}^{1}(N) & \theta_{E}^{1}(A) & \theta_{I}^{1} & 1
\end{array}
\end{aligned}
$$

Figure 2: The effect of prohibiting acquisitions on project variety.

the absence of an innovation effect is that $\pi_{I}(L, 0)-\pi_{I}(\ell, 0) \geq \pi_{E}(L, \ell)$, so that a nondrastic innovation would increase incumbent monopoly profits by a large amount, while the entrant's profit under duopoly competition has to be relatively low (competition is intense or biased against the entrant). We further show that, once this profit condition holds, the innovation effect will be zero if commercialization costs $\kappa$, the entrant's bargaining power $\beta$ and the probability $p$ of a drastic innovation are sufficiently low.

\subsection{The Size of the Effect on Variety}

As an input into our subsequent policy discussion, we analyze how the market environment determines the size of the innovation-reducing effect of restricting acquisitions.

Proposition 3. Consider any equilibrium under a laissez-faire policy $\left(r_{I}^{A}, r_{E}^{A}\right)$ and any equilibrium under the no-acquisition policy $\left(r_{I}^{N}, r_{E}^{N}\right)$.

(i) The size of the policy effect $\Delta_{\mathcal{V}}$ is (a) weakly increasing in entrant bargaining power $\beta$, (b) weakly decreasing in the incumbent's profits under competition $\pi_{I}(\ell, L)$ and (c) strictly decreasing in the entrant's profits under competition $\pi_{E}(L, \ell)$ if $\theta_{I}^{1}<\theta_{E}^{1}(N)$, but weakly increasing if $\theta_{E}^{1}(N)<\theta_{I}^{1}$.

(ii) The effects in (i) are strict if $\theta_{I}^{1}<\theta_{E}^{1}(A)$ and they are zero if $\theta_{I}^{1}>\theta_{E}^{1}(A)$.

This result identifies the circumstances under which the innovation effect is important. To understand it, recall that in both policy regimes the variety of research projects is determined by the most expensive project any firm is willing to invest in, so that $\Delta_{\mathcal{V}}=$ $\max \left\{\theta_{E}^{1}(A), \theta_{I}^{1}\right\}-\max \left\{\theta_{E}^{1}(N), \theta_{I}^{1}\right\}$. Thus, the effect of a parameter on the loss of variety is equivalent to its effect on the difference between these critical projects.

An increase in the entrant's bargaining power $\beta$ increases his payoff in case of an 
acquisition and thus $\theta_{E}^{1}(A) .{ }^{28}$ The change neither affects $\theta_{E}^{1}(N)$ (since acquisitions are not allowed) nor $\theta_{I}^{1}$ (since there is no acquisition if the entrant does not innovate). Combining these observations, for orderings (I) and (II), an increase in $\beta$ strictly increases $\Delta_{\mathcal{V}}$, as it increases $\theta_{E}^{1}(A)$ without affecting $\theta_{E}^{1}(N)$ and $\theta_{I}^{1}$. For ordering (III), an increase in $\beta$ has no effect, as it does not change $\theta_{I}^{1} \cdot{ }^{29}$ Next, an increase in the incumbent's profits under competition $\pi_{I}(\ell, L)$ neither affects $\theta_{E}^{1}(N)$ nor $\theta_{I}^{1}$, but it reduces the acquisition surplus and therefore decreases $\theta_{E}^{1}(A)$. The overall effect is a strict reduction in $\Delta_{\mathcal{V}}$ for orderings (I) and (II), and no effect for ordering (III). Finally, the effect of an increase in the entrant's duopoly profit $\pi_{E}(L, \ell)$ is more subtle, because $\pi_{E}(L, \ell)$ increases both $\theta_{E}^{1}(A)$ and $\theta_{E}^{1}(N)$, but the increase is greater for $\theta_{E}^{1}(N)$. As ordering (III) may arise only when the acquisition is genuine, these arguments again highlight the importance of distinguishing these two cases.

To summarize, Proposition 3 shows how the loss of variety depends on bargaining power and the intensity of potential competition as captured by duopoly profits. This result is a useful ingredient in the policy analysis, as it identifies circumstances in which competition authorities can implement a more restrictive acquisition policy without substantial negative effects on innovation. We provide a more detailed policy discussion in Section 8.

\subsection{The Effect on Duplication}

The acquisition policy not only affects variety and thereby the probability of innovation, but also the firms' incentives to duplicate research projects. Contrary to the laissez-faire case, duopolistic competition arises after a non-drastic innovation of the entrant. This affects the critical values.

Corollary 2. Prohibiting acquisitions increases the incumbent's duplication incentives and decreases those of the entrant, that is: (i) $\theta_{I}^{2}(N)>\theta^{2}(A)$ and (ii) $\theta^{2}(A)>\theta_{E}^{2}(N)$.

Intuitively, (i) if the entrant invests in a project, the incumbent gains more from duplicating it under a no-acquisition policy than under laissez-faire: Without the acquisition option, own investments that duplicate entrant's research are the only means of preventing competitive entry. As to the entrant, (ii) duplicating the incumbent's investments is less attractive under the no-acquisition policy than under laissez-faire because of the absence of prospective gains from selling the firm. Since the incentives for duplication increase for the incumbent and decrease for the entrant, the overall amount of duplication can

\footnotetext{
${ }^{28}$ While we do not model the sources of bargaining power explicitly, the analysis of Gans and Stern (2000) suggests that it could, for instance reflect intellectual property rights.

${ }^{29}$ This argument and the one in the next paragraph apply when orderings (II) and (III) are strict. When $\theta_{I}^{1}$ is equal to one of the entrant's critical projects, matters are more subtle, but the intuition is similar. See the proof for details.
} 
either increase or decrease. We discuss the complex net effects of these policy reactions in Proposition B.4 in Online Appendix B.3.4.

By investigating $R \& D$ portfolios rather than just total $R \& D$ efforts, we can distinguish between innovation investments for duplicative projects and new projects. The assumption that the incumbent can patent her innovation makes acquisitions and innovations substitutes for the incumbent. Thus, prohibiting acquisitions increases incumbent R\&D effort in a model where the effort choice is one-dimensional, but the above analysis shows that this increase is driven exclusively by duplication incentives which do not translate into an increase in innovation probability. A model with a one-dimensional effort choice cannot capture such strategic project choices, see Appendix B.7. Finally, this is important beyond the theoretical analysis of start-up acquisitions because it also shows that considering total R\&D efforts to evaluate the impact of a policy on innovation can be misleading. If a policy increases duplication, total R\&D spending may increase while the overall innovation probability decreases.

\section{$6 \quad$ Alternative Policies}

Preventing incumbents from acquiring start-ups who produce close substitutes can potentially foster competition, but it may hurt innovation. The above analysis suggests that there are circumstances in which it is beneficial to intervene, but translating these circumstances into criteria which are readily applicable for competition authorities is non-trivial. In the following, we therefore analyze alternative policies that are not contingent on details of the environment, but nevertheless not as crude as outright prohibitions, as their intensity can be adjusted to societal preferences. Even though the effects of these policies differ in detail, they share the common attractive feature that they only prevent acquisitions of entrants who would obtain relatively high stand-alone profits, suggesting they would be viable competitors. Moreover, three of the four policies lead to innovation outcomes that are between those under laissez-faire and a no-acquisition policy, respectively.

\subsection{Behavioral Remedies}

Rather than prohibiting acquisitions completely, competition agencies often impose remedies on the acquiring firm. We consider two possible approaches. The first approach only affects genuine acquisitions and has no effect on killer acquisitions, whereas the second approach only has a bearing on killer acquisitions. 


\subsubsection{Restrictions on Technology Usage}

A behavioral remedy could inhibit the use of the startup's technology by the incumbent. For instance, the EU only accepted Google's recent acquisition of Fitbit conditional on licensing requirements and limitations on data usage. Such measures presumably have adverse profit effects on the acquiring firm when the acquisition is genuine. To capture this, we assume that the incumbent's market profit after an acquisition and commercialization of technology $L$ is $\rho \pi_{I}(L, 0)$ where $\rho \in[0,1)$. Lower $\rho$ implies more stringent remedies, with $\rho=1$ corresponding to a laissez-faire policy. The remedy only affects business operations that are related to the acquired technology, so that the incumbent can use her existing technology $\ell$ without restrictions. Thus, independently of $\rho$, the incumbent can secure herself a post-acquisition market profit of at least $\pi_{I}(\ell, 0)$. If the incumbent discovers technology $L$ herself, there is no reduction in market profits, as remedies are only imposed in case of an acquisition. Denote with $\theta_{i}^{k}(\rho)$ the critical value $k \in\{1,2\}$ of firm $i$ when the remedy is $\rho \in[0,1)$. We now characterize the effects of imposing such a remedy.

Proposition 4 (Restrictions on technology usage). In the killer-acquisition case, restrictions on technology usage have no effect. In the genuine-acquisition case,

(i) If $\pi_{I}(\ell, 0) \leq \pi_{E}(L, \ell)-\kappa+\pi_{I}(\ell, L)$ and $\rho \leq \frac{\pi_{E}(L, \ell)+\pi_{I}(\ell, L)}{\pi_{I}(L, 0)}$, then all critical values are identical to those under a prohibition and the incumbent never acquires the entrant.

(ii) Otherwise, the critical values lie between those with laissez-faire and prohibition of acquisitions and the incumbent acquires the entrant with an $L$ innovation. If $\pi_{I}(\ell, 0)>\pi_{E}(L, \ell)-\kappa+\pi_{I}(\ell, L)$ and $\rho \leq \frac{\pi_{I}(\ell, 0)+\kappa}{\pi_{I}(L, 0)}$, then, in contrast to the case without remedies, the incumbent does not commercialize the innovation after acquisitions.

(i) shows that strong remedies prevent genuine acquisitions of entrants with high standalone profits. For entrants with low stand-alone profits (ii), the remedy leads to innovation strategies that are between those under prohibition and those under laissez-faire. Apart from not addressing killer acquisitions, remedies have another negative effect: They turn some genuine acquisitions into killer acquisitions when the incumbent's benefit from commercializing technology $L$ is not too much larger than $\kappa$, so that, with the remedies, commercializing technology $L$ is no longer worthwhile.

\subsubsection{Prohibition of "killing"}

Alternatively, competition policy could prevent incumbents from shutting down acquired entrants. ${ }^{30}$ Such a remedy would cause the incumbent to forgo some acquisitions that she

\footnotetext{
${ }^{30}$ In practice, this would require competition authorities to conduct ex-post reviews to evaluate whether shutting down would constitute a monopolization/abuse of dominance offence. Although not
} 
would otherwise pursue. Our next result characterizes the innovation effect of this policy; we use $\theta_{i}^{k}(P K)$ for the critical values when killing is prohibited.

Proposition 5 (Prohibition of "killing"). In the genuine-acquisition case, prohibition of killing has no effect. In the killer-acquisition case,

(i) If $\pi_{I}(L, 0)-\pi_{I}(\ell, L) \leq \pi_{E}(L, \ell)$, then all critical values are identical to those under a prohibition, and the incumbent never acquires the entrant.

(ii) Otherwise, the critical values lie between those with laissez-faire and prohibition of acquisitions. The incumbent acquires the entrant with the $L$ innovation, but, in contrast with the case without remedies, commercializes the innovation.

When the policy has an innovation effect, it resembles the previous remedy: It prevents acquisitions of entrants with high stand-alone profits (i), and, for entrants with low standalone profits, it leads to innovation strategies between those under prohibition and those under laissez-faire (ii). Clearly, (killer) acquisitions that are not prevented are turned into genuine acquisitions, which may be the primary intention behind such a policy.

\subsection{Fiscal Policies}

The goal of inducing start-ups to compete with instead of sell out to the incumbent could be achieved by fiscal policies, as suggested by Lemley and McCreary (2020), who group such policies into "sticks" and "carrots". "Sticks" reduce the profitability of acquisitions, while "carrots" aim at increasing the profitability of market entry for start-ups. We consider one specific policy of each type and show that, even though these policies may affect acquisition incentives in a similar way, there are important differences in ex-ante innovation effects.

\subsubsection{Taxing Acquisitions}

One specific "stick" affecting the relative profitability of acquisitions and market entry is an acquisition $\operatorname{tax} \tau$. Starting from a pre-tax value which we denote as $\bar{B}$, the bargaining surplus falls to some $B^{\tau}$ as a result of the tax. We show how the critical values $\theta_{i}^{k}(\tau)$ under a tax $\tau$ depend on the characteristics of the start-up and their technology:

Proposition 6 (Taxing acquisitions).

(i) If $\tau \geq \frac{\bar{B}}{\pi_{E}(L, \ell)-\kappa}$, then all critical values are identical to those under a prohibition and the incumbent never acquires the entrant.

common, this is sometimes done. For instance, after Mallinckrodt's subsidiary Questcor acquired the rights for Synacthen from Novartis, the FTC successfully took the firm to court for anti-competitive behavior, which was manifest in excessive prices (https://www.ftc.gov/system/files/documents/cases/ 170118mallinckrodt_complaint_public.pdf). For a broader discussion of conceivable policy responses, see OECD (2020). 
(ii) If $0<\tau<\frac{\bar{B}}{\pi_{E}(L, \ell)-\kappa}$ then the critical values lie between those under the laissezfaire and the no-acquisition policy and the incumbent acquires the entrant with the $L$ innovation.

Intuitively, (i) the tax is equivalent to a prohibition of acquisitions if it is so high that even at the minimal acceptable acquisition price (which is equal to the entrant's outside option), the tax bill would be higher than the bargaining surplus. This happens when $\pi_{E}(L, \ell)-\kappa$, the entrant's profit net of commercialization cost, is high. By contrast (ii), for lower taxes acquisitions of entrants with technology $L$ still take place. The firms' innovation strategies are affected in the same direction, but not to the same extent as under a prohibition of acquisitions. Therefore, compared with a prohibition, a tax results in a smaller negative innovation effect for entrants with low stand-alone profits, but it only prevents acquisitions of entrants with high stand-alone profits.

\subsubsection{Increasing Profitability of IPOs}

As an alternative to acquisition taxes, Lemley and McCreary (2020) suggest "carrot" policies to make initial public offerings (IPOs) more attractive, such as lower taxes on IPO gains or a quicker and more straightforward IPO process. ${ }^{31}$ We operationalize such policies by supposing that the net profit of the entrant is given by $\eta \pi_{E}(H)$ and $\eta \pi_{E}(L, \ell)$, where $\eta=1$ represents the status quo and $\eta>1$ represents the preferential IPO policy. For $i \in\{I, E\}$ and $k \in\{1,2\}$, denote the critical value when the IPO policy is $\eta>1$ with $\theta_{i}^{k}(\eta)$. The following result shows the effect of such a policy.

Proposition 7 (Increasing Profitability of IPOs). Consider an IPO policy $(\eta>1)$.

(i) All critical values lie above those under the laissez-faire policy.

(ii) The incumbent acquires the entrant with the $L$ innovation if and only if $\eta<\frac{\bar{B}+\pi_{E}(L, \ell)}{\pi_{E}(L, \ell)}$.

According to (i), lower IPO taxes would increase the entrant's incentives to invest in both variety and duplication of $\mathrm{R} \& \mathrm{D}$. This is not surprising, as more profitable IPOs increase the entrant's payoffs no matter whether an acquisition takes place or not. By (ii), similarly to an acquisition tax and behavioral remedies, a preferential IPO treatment would prevent acquisitions of entrants with high stand-alone profits. A more subtle effect of preferential IPO treatment is that it increases the incumbent's incentive to duplicate R\&D projects. Since the entrant's duplication incentives increase as well, preferential IPO treatment would unambiguously increase research duplication.

\footnotetext{
${ }^{31}$ Companies already try to avoid the complicated IPO process by merging with blank-cheque companies known as Spacs. The number of such deals has exploded in 2020 and 2021 to potentially worrying levels. For example, see "Spac boom eclipses 2020 fundraising record in single quarter", O. Aliaj and A. Kasumov, Financial Times, March 17, 2021, (https://www.ft.com/content/321400c1-9c4d-40ac-b464-3a64c1c4ca80).
} 


\section{Robustness}

We now provide robustness results. Appendix B.5 contains formal results and proofs.

Uncertainty at the Time of Acquisition In our model, before entering acquisition negotiations, both firms know whether the innovation is drastic or not. In practice, this may be difficult: Extensive testing may be necessary to identify cost savings or quality improvements. We now show that the effects of prohibiting an acquisition remain similar if the technology level of an innovation is uncertain at the time of the acquisition. We maintain the setting of Section 3, but assume that only the correct project is revealed at the end of the investment stage, not its technology level. Thus, interim technology states $\left(t_{I}^{\text {int }}, t_{E}^{i n t}\right) \in\{(0,0),(0,1),(1,0)\}$ are realized, where 1 indicates that the firm received a patent and 0 indicates that it did not. After the acquisition stage, the technology level of the correct project is realized as $L$ or $H$. Thereafter, firms decide on commercialization, before the final technology states $\left(t_{I}^{f i n}, t_{E}^{f i n}\right) \in \mathcal{T}$ are realized. Everything else remains as before. Proposition B.5 in Appendix B.5.1 shows that, irrespective of the policy regime, uncertainty does not affect equilibrium investments and thus does not change the policy effect. However, uncertainty does influence the frequency of acquisitions. The incumbent will acquire the entrant irrespective of the technology level of the latter's innovation because the expected surplus at the time is positive, since it is a convex combination of a positive acquisition surplus with the $L$ technology and no acquisition surplus with the $H$ technology.

Asymmetric Chances of Receiving Patents We show that the variety of pursued investment projects is invariant to the assumption that firms are equally likely to receive the patent after simultaneous discovery. Let the probability of receiving the patent be $\alpha_{I} \in(0,1)$ for the incumbent and $\left(1-\alpha_{I}\right)$ for the entrant. ${ }^{32}$ Proposition B.6 in Appendix B.5.2 shows that, regardless of $\alpha_{I}$, banning acquisitions weakly reduces the variety of research projects and thereby the innovation probability. Furthermore, the size of the policy effect is independent of $\alpha_{I}$. Therefore, the results on the relation between parameters and the size of the policy effect identified in Proposition 3 are also robust to changes in $\alpha_{I}$. This holds because $\alpha_{I}$ matters only when both firms discover an innovation. Thus, it affects duplication incentives, but not the incentives to invest in projects in which the competitor is not investing. Since variety is given by $\max \left\{\theta_{E}^{1}, \theta_{I}^{1}\right\}$, it is not affected by $\alpha_{I}$ in either policy regime, so that the size of the policy effect does not depend on $\alpha_{I}$.

Heterogeneous Commercialization Costs Due to a better infrastructure or a more developed sales network, the incumbent might be able to commercialize the inno-

\footnotetext{
${ }^{32}$ The main model corresponds to $\alpha_{I}=1 / 2$.
} 
vation at a lower cost $\kappa_{I}$ than the entrant $\left(\kappa_{E}\right)$. Adjusting Assumption 2, we suppose $\pi_{E}(L, \ell) \geq \kappa_{E}$ and $\pi(H)-\pi_{I}(\ell, 0) \geq \kappa_{I}$. We focus on the killer-acquisition case, so that $\pi_{I}(L, 0)-\pi_{I}(\ell, 0)<\kappa_{I}$. We add the innocuous assumption that $\pi_{E}(L, \ell) \leq \pi_{I}(L, 0)$, requiring that, with an $L$-technology, the monopolist would obtain market profits at least as high as the entrant would from competing against technology $\ell{ }^{33}$ Proposition B.7 in Appendix B.5.3 shows that banning acquisitions reduces the variety of research projects, which tends to reduce the innovation probability. A prohibition of acquisitions now results in an additional inefficiency, as it forces the entrant to commercialize the H-technology using the cost $\kappa_{E}$ instead of letting the incumbent commercialize it at the lower cost $\kappa_{I}$.

Heterogeneous Innovation Outcomes Costly projects might be more innovative and thus yield a drastic innovation with a larger probability. Our results are robust to allowing for such heterogeneity. We now suppose the probability of a drastic innovation $p(\theta)$ is an increasing function of $\theta, p:[0,1) \rightarrow[0,1)$, which is continuous, differentiable and concave. Keeping all remaining assumptions as in the main model, Proposition B.8 in Appendix B.5.4 establishes that prohibiting acquisitions still reduces variety and innovation probability. While the effects refer to any innovation, the fact that, as before, banning acquisitions induces entrants to stop investing in the most expensive projects in their portfolio, gives the result an interesting twist: Banning acquisitions will reduce drastic innovations relatively more compared to non-drastic innovations. Hence, in addition to reducing the overall innovation probability, banning acquisitions will change the direction of the remaining projects away from drastic innovation.

Multiple Entrants We now sketch why allowing for multiple entrants should not change the effects of a restrictive acquisition policy on innovation substantially, without going into details of equilibrium existence and characterization. We focus on the killeracquisition case, assuming there are two entrants. Compared with the main model, the analysis changes mainly because firms need to consider that two competitors might invest in some project, which reduces the probability of obtaining a patent. To capture the willingness to invest in such projects, we define critical projects $\theta_{i}^{3}$ in a similar way as $\theta_{i}^{1}$ and $\theta_{i}^{2}$. Clearly, $\theta_{i}^{3}<\theta_{i}^{2}$, reflecting the lower probability of obtaining a patent when three rather than two firms invest. Crucially, the number of entrants does not affect $\theta_{i}^{1}$ and $\theta_{i}^{2}$. Therefore, the highest critical value is still $\theta_{E}^{1}$, no matter which policy regime applies. Moreover, in any equilibrium, for any project $\theta \leq \theta_{E}^{1}$ at least one firm invests a positive amount. Thus, as in the main model, the entrants' critical projects determine variety. Therefore, the policy effect on variety remains the same with multiple entrants as with a single entrant.

\footnotetext{
${ }^{33}$ We do not rely on this natural assumption in the main model, which is why we only add it here.
} 


\section{Policy Discussion}

Merger analysis usually weighs potential efficiency gains against the reduction in competition. In this section, we focus on the trade-off between ex-post competition and ex-ante innovation effects instead, while acknowledging that other merger efficiencies may also exist. Though we did not make the effects of competition on consumer surplus explicit in the above analysis, we will base the following discussion on the innocuous assumption that, for any fixed technology level, consumers benefit from entry. ${ }^{34}$

We start by noting that the trade-off is absent in some situations. Aside from the trivial case that the pro-competitive arguments for a prohibition will be absent if the entrant needs the incumbent to commercialize the innovation (which we ruled out by Assumption 2), we identified the more interesting possibility that a prohibition has no adverse innovation effects. As discussed at the end of Section 5.1, a necessary condition for a zero innovation effect is that a non-drastic innovation would result in a large increase of the incumbent's monopoly profit, while the entrant's duopoly profit is low (competition is intense or biased against the entrant). This condition appears plausible in an industry where the incumbent benefits from network effects, making it hard for the entrant to stand on his own feet. Once this profit condition holds, the innovation effect will be zero if commercialization costs, the entrant's bargaining power and the probability of a drastic innovation are sufficiently low. Then, the anti-competitive effect of an acquisition suffices to justify an intervention. Even when the prohibition of acquisitions impedes innovations, this is not always detrimental to consumers: Under the conditions of the killer-acquisition case (high commercialization cost and low effects of the innovation on monopoly profits), if the chances of a drastic innovation are negligible $(p=0)$, any innovation in the laissez-faire case would be nondrastic and would therefore never reach the market. Prohibiting acquisitions is thus clearly justified.

When there is an innovation effect, the trade-off depends on policy objectives and the market environment in a subtle way. Proposition 3 illustrates the conditions influencing the size of the innovation effect. For instance, it implies that, in the killer-acquisition case, an exogenous reduction in the entrant's duopoly profits $\pi_{E}(L, \ell)$ increases the size of the adverse innovation effect. However, low entrant profits may reflect more intense competitive interaction between the firms and therefore a higher consumer surplus relative to the monopoly case. Thus, the gains from maintaining competition might also be particularly high in this case.

In Appendix B.6, we discuss these trade-offs in more detail, using standard differentiated Bertrand and Cournot models. The analysis suggests that, from a consumer perspective, the net gains from prohibiting acquisitions tend to become smaller as the

\footnotetext{
${ }^{34}$ See Section B.6 for a precise formalization of this assumption.
} 
entrant's bargaining power and the intensity of competition (as captured by the degree of substitution) increase; however only in the Cournot example do they ever become negative.

One might therefore conclude that competition authorities should intervene selectively, depending on market characteristics. However, doing so would require precise information, which the agencies might lack. Some of the alternative policies discussed in Section 6 might be advantageous in this respect. Importantly, these policies would prevent acquisitions only in those circumstances when an entrant would earn substantial stand-alone profits, suggesting that he would be a viable competitor. There are pitfalls, however. Remedies that limit the profits that an incumbent can obtain by using the entrant's technology are potentially problematic as they do not address the problem of killer acquisitions and, in some cases, even transform genuine acquisitions into killer acquisitions. Prohibiting the "killing" turns killer into genuine acquisitions, but similarly decreases innovation incentives and may be difficult to enforce. A more promising approach would be a tax on acquisitions, which would be easier to implement than prohibiting "killing" while still preventing acquisitions of entrants with high stand-alone value. Another promising policy would be to increase profitability of IPOs, perhaps through lower taxes on IPO profits. Unlike other policies we discussed, this would increase incentives to innovate. ${ }^{35}$ Finally, since these policies are not mutually exclusive, a combination of policies (for example, a tax on acquisitions and a lower tax on IPO profits) could result in a better overall outcome than any single policy. Our model provides a framework in which the effects of such combined policies can be analyzed.

Obviously, this policy discussion is limited by the assumptions of our framework. For example, we have not treated the possibility that there are multiple incumbents, which could lead to the possibility that firms acquire entrants to avoid that competitors have access to their technology. Further, our analysis does not directly apply to the interesting case where an incumbent in one market acquires a start-up that has recently entered a related market which the incumbent cannot serve with her existing technology. Moreover, our approach focuses on the short-run policy effects. In the long term, rather than merely killing a potential entrant, the incumbent can combine the knowledge of the two firms to expand its technological lead, which is likely to make entry ever more difficult. It would be interesting to analyze how incumbents and potential entrants target their innovation activities when entry can take place repeatedly and the incumbent's technology improves as a result of acquisitions. Is increasing dominance of the incumbent an inevitable outcome? Will the innovation process eventually slow down because it becomes too hard for entrants to compete? While these questions are beyond the scope of the current paper, our analysis suggests that to answer them it would be expedient to take the policy effects on project choice into account, rather than only the effects on the overall innovation level.

\footnotetext{
${ }^{35}$ Of course, a possible cost of such a policy is that it leads to lower tax revenues.
} 


\section{Conclusion}

Recently, there has been a heated debate on the policy towards start-up acquisitions, with particular emphasis on innovation effects. Motivated by this discussion, we provide a theory of the strategic choice of innovation projects by incumbents and start-ups which allows for endogenous acquisition and commercialization decisions. We use this framework for a policy analysis. We first find that prohibiting start-up acquisitions weakly reduces the variety of research projects pursued and thereby the probability of discovering innovations, and that it may induce the incumbent to strategically duplicate the entrant's projects to prevent competition. However, our analysis shows that the negative innovation effect of prohibiting acquisitions may well be absent for innovations with high commercialization potential. Even for less attractive innovations that the incumbent would not want to commercialize, the adverse innovation effects may be negligible, for instance, if the entrant has low bargaining power and the incumbent's duopoly profits are high, so that the competition-enhancing effect of prohibiting acquisitions is likely to dominate in this case. However, an approach that conditions on details of the market environment is arguably impractical, as it imposes heavy informational requirements on competition authorities. Our analysis suggests that a useful alternative might be to rely on policies that weaken the incentives for acquisitions, while leaving the details to the market. Suitable remedies, acquisition taxes and preferable treatments of IPOs would make sure that acquisitions only arise in marginal cases where the entrant would not be very strong on its own. 


\section{A Appendix}

The proofs of our equilibrium characterizations for the laissez-faire case and the noacquisition policy rely on one common ingredient that applies in the killer acquisitions case as well as the genuine acquisition case. The logic of this crucial result also underlies the arguments for the results on alternative policies in Section 6 and the extensions in Section 7. In view of its fundamental importance, we state and discuss this result. Its proof is in Appendix B.

Proposition A.1. Any equilibrium under laissez-faire or the no-acquisition policy must satisfy (a)-(f) below. If (a)-(f) all hold, the investment functions $r_{E}(\theta)$ and $r_{I}(\theta)$ can be sustained as an equilibrium.

(a) $r_{E}(\theta)=1$ and $r_{I}(\theta)=1$ whenever $\theta \in\left[0, \theta_{E}^{2}\right]$

(b) $r_{E}(\theta)=0$ and $r_{I}(\theta)=0$ whenever $\theta \in\left(\max \left\{\theta_{I}^{1}, \theta_{E}^{1}\right\}, 1\right)$

(c) $r_{E}(\theta)=1$ and $r_{I}(\theta)=0$ whenever $\theta \in\left(\max \left\{\theta_{I}^{2}, \theta_{I}^{1}\right\}, \theta_{E}^{1}\right]$

(d) $r_{E}(\theta)=\frac{C\left(\theta_{I}^{1}\right)-C(\theta)}{C\left(\theta_{I}^{1}\right)-C\left(\theta_{I}^{2}\right)}$ and $r_{I}(\theta)=\frac{C\left(\theta_{E}^{1}\right)-C(\theta)}{C\left(\theta_{E}^{1}\right)-C\left(\theta_{E}^{2}\right)}$ whenever $\theta \in\left(\max \left\{\theta_{I}^{1}, \theta_{E}^{2}\right\}, \min \left\{\theta_{E}^{1}, \theta_{I}^{2}\right\}\right]$.

(e) Firms use an anti-coordination profile whenever $\theta \in\left(\theta_{I}^{2}, \min \left\{\theta_{I}^{1}, \theta_{E}^{1}\right\}\right]$

(f) The equilibrium satisfies $r_{E}(\theta)=0$ and $r_{I}(\theta)=1$ in all other cases.

The result characterizes equilibrium choices $r_{i}(\theta)$ on the basis of the relation of that project $\theta$ to the critical projects $\theta_{E}^{1}, \theta_{I}^{1}, \theta_{E}^{2}$ and $\theta_{I}^{2}$. It leads to necessary and sufficient conditions for the equilibrium. In line with Proposition 1, Parts (a) and (b) show that there is full investment in sufficiently cheap projects and no investment in sufficiently expensive projects. In the remaining constellations, intermediate situations arise, with investment by at least one firm, but not full investment by both. The details of the equilibrium structure then depend on the order of the critical values. As we have seen in Proposition 1, there are several conceivable orders for the laissez-faire case. These orders differ from those that are possible under a no-acquisition policy (Propositions B.1 and B.2). Using Proposition A.1, it is straightforward to show how these differences in orders translate into differences in equilibrium structures. 


\section{References}

Bardey, David, Bruno Jullien, and Jean-Marie Lozachmeur, "Health insurance and diversity of treatment," Journal of Health Economics, 2016, 47, 50-63.

Bavly, Gilad, Yuval Heller, and Amnon Schreiber, "Social Welfare in Search Games with Asymmetric Information," Mimeo, 2020.

Bourreau, Marc and Alexandre de Streel, Digital Conglomerates and EU Competition Policy, Paris, Namur: Télecom Paris-Tech, Université de Namur, 2019.

_ , Bruno Jullien, and Yassine Lefouili, "Mergers and Demand-Enhancing Innovation," TSE Working Papers No. 18-90\%, 2019.

Bryan, Kevin A. and Erik Hovenkamp, "Antitrust Limits on Startup Acquisitions," Review of Industrial Organization, 2020.

_ and _ , "Startup acquisitions, error costs, and antitrust policy," The University of Chicago Law Review, 2020, 87 (2), 331-356.

- and Jorge Lemus, "The Direction of Innovation," Journal of Economic Theory, 2017, 172, 247-272.

Cabral, Luís, "Standing on the Shoulders of Dwarfs: Dominant Firms and Innovation Incentives," CEPR Discussion Papers No. 13115, 2018.

_ , "Merger policy in digital industries," Information Economics and Policy, 2020.

Callander, Steven, "Searching and learning by trial and error," American Economic Review, 2011, 101 (6), 2277-2308.

- and Niko Matouschek, "The Novelty of Innovation: Competition, Disruption, and Antitrust Policy," Unpublished Draft, 2021. March 31, 2021.

Carnehl, C. and J. Schneider, "A Quest for Knowledge," Unpublished Draft, 2021. June, 2021.

Crémer, Jacques, Yves-Alexandre de Montjoye, and Heike Schweitzer, "Competition policy for the digital era," Report for the European Commission, 2019.

Cunningham, Colleen, Florian Ederer, and Song Ma, "Killer acquisitions," Journal of Political Economy, 2021, 129 (3), 649-702.

Denicolò, Vincenzo and Michele Polo, "Duplicative research, mergers and innovation," Economics Letters, 2018, 166, 56-59.

Dijk, Esmee, Jose L. Moraga-Gonzalez, and Evgenia Motchenkova, "How Do Start-up Acquisitions Affect the Direction of Innovation?," Unpublished Draft, 2021. January 3, 2021.

Federico, Giulio, Gregor Langus, and Tommaso Valletti, "A simple model of mergers and innovation," Economics Letters, 2017, 15\%, 136-140.

_ , _ , and _ , "Horizontal mergers and product innovation," International Journal of Industrial Organization, 2018, 59, 1-23. 
Fumagalli, Chiara, Massimo Motta, and Emanuele Tarantino, "Shelving or developing? The acquisition of potential competitors under financial constraints," Mimeo, 2020.

Furman, Jason, Diane Coyle, Amelia Fletcher, Derek McAuley, and Philip Marsden, "Unlocking digital competition: Report of the digital competition expert panel," UK government publication, HM Treasury, 2019.

Gans, J, D.H. Hsu, and Scott Stern, "When does start-up innovation spur the gale of creative destruction?," The RAND Journal of Economics, 2002, 33 (4).

Gans, Joshua S and Scott Stern, "Incumbency and R\&D incentives: Licensing the gale of creative destruction," Journal of Economics \& Management Strategy, 2000, 9 (4), 485-511.

Gautier, Axel and Joe Lamesch, "Mergers in the digital economy," CESifo Working Paper No. 8056, 2020.

Gilbert, Richard and Michael L. Katz, "Dynamic Merger Policy and Pre-Merger Investment: Equilibrium Product Choice by an Entrant," Available at SSRN 3795782, 2021.

Gilbert, Richard J. and David M.G. Newbery, "Preemptive patenting and the persistence of monopoly," The American Economic Review, 1982, pp. 514-526.

Gilbert, R.J., "Competition, Mergers, and R\&D Diversity," Review of Industrial Organization, 2019, pp. 465-484.

Hollenbeck, Brett, "Horizontal mergers and innovation in concentrated industries," Quantitative Marketing and Economics, 2020, 18 (1), 1-37.

Hovenkamp, Herbert and Carl Shapiro, "Horizontal mergers, market structure, and burdens of proof," Yale LJ, 2017, 127, 1996.

Kamepalli, Sai Krishna, Raghuram Rajan, and Luigi Zingales, "Kill Zone," Stigler Center for the Study of the Economy and the State, 2020.

Katz, Michael L, "Big-Tech Mergers: Innovation, Competition for the Market, and the Acquisition of Emerging Competitors," Unpublished Draft, 2020.

Lee, Tom and Louis L Wilde, "Market structure and innovation: A reformulation," The Quarterly Journal of Economics, 1980, 94 (2), 429-436.

Leiner, Barry M, Vinton G Cerf, David D Clark, Robert E Kahn, Leonard Kleinrock, Daniel C Lynch, Jon Postel, Larry G Roberts, and Stephen Wolff, "A brief history of the Internet," ACM SIGCOMM Computer Communication Review, 2009, 39 (5), 22-31.

Lemley, Mark A. and Andrew McCreary, "Exit strategy," Mimeo, 2020.

Letina, Igor, "The road not taken: competition and the R\&D portfolio," The RAND Journal of Economics, 2016, 47 (2), 433-460.

- and Armin Schmutzler, "Inducing variety: A theory of innovation contests," 
International Economic Review, 2019, 60 (4), 1757-1780.

Loury, Glenn C., "Market structure and innovation," The Quarterly Journal of Economics, 1979, 93 (3), 395-410.

Marshall, Guillermo and Alvaro Parra, "Innovation and Competition: The Role of the Product Market," International Journal of Industrial Organization, 2019, 65, $221-247$

Mason, Robin and Helen Weeds, "Merger policy, entry, and entrepreneurship," European Economic Review, 2013, 57, 23-38.

Mermelstein, Ben, Volker Nocke, Mark A Satterthwaite, and Michael D Whinston, "Internal versus external growth in industries with scale economies: A computational model of optimal merger policy," Journal of Political Economy, 2020, 128 (1), 301-341.

Moraga-González, José-Luis, Evgenia Motchenkova, and Saish Nevrekar, "Mergers and Innovation Portfolios," CEPR Discussion Paper No. 14188, 2019.

Morton, Fiona Scott, Pascal Bouvier, Ariel Ezrachi, Bruno Jullien, Roberta Katz, Gene Kimmelman, A Douglas Melamed, and Jamie Morgenstern, "Committee for the study of digital platforms: Market structure and antitrust subcommittee report," Chicago: Stigler Center for the Study of the Economy and the State, University of Chicago Booth School of Business, 2019.

Motta, Massimo and Emanuele Tarantino, "The effect of horizontal mergers, when firms compete in investments and prices," Mimeo, 2018.

- and Martin Peitz, "Big tech mergers," Information Economics and Policy, 2021, 54, 100868.

_, Sandro Shelegia et al., "The kill zone: copying, acquisition and start-ups direction of innovation," Technical Report 2021.

Norbäck, Pehr-Johan and Lars Persson, "Entrepreneurial innovations, competition and competition policy," European Economic Review, 2012, 56, 488-506.

OECD, "Start-Ups, Killer Acquisitions and Merger Control - A Background Note," 2020.

Phillips, Gordon M. and Alexei Zhdanov, "R\&D and the Incentives from Merger and Acquisition Activity," The Review of Financial Studies, 2013, 26 (1), 34-78.

Rasmusen, Eric, "Entry for Buyout," The Journal of Industrial Economics, 1988, 36 (3), 281-299.

Reinganum, Jennifer F, "Uncertain innovation and the persistence of monopoly," The American Economic Review, 1983, 73 (4), 741-748.

Salop, Steven, "Modifying merger consent decrees: an economist plot to improve merger enforcement policy," Antitrust, 2016, pp. 15-20.

Salop, Steven C. and Carl Shapiro, "Whither Antitrust Enforcement in the Trump Administration?," Antitrust Source, 2017. 
Schmutzler, Armin, "Competition and investment-A unified approach," International Journal of Industrial Organization, 2013, 31 (5), 477-487.

Segal, Ilya and Michael D. Whinston, "Antitrust in innovative industries," American Economic Review, 2007, 97 (5), 1703-1730.

Shapiro, Carl, "Antitrust in a Time of Populism," International Journal of Industrial Organization, 2018, 61, 714-748.

Vives, Xavier, "Innovation and Competitive Pressure," The Journal of Industrial Economics, 2008, 56 (3), pp. 419-469.

Wickelgren, Abraham L., "Optimal merger standards for potential competition: the effect of ex ante investment incentives," Unpublished Draft, 2021. February, 2021.

Yi, Sang-Seung, "Market structure and incentives to innovate: the case of Cournot oligopoly," Economics Letters, 1999, 65 (3), 379-388. 


\section{B Appendix (For Online Publication)}

This online appendix provides proofs of our most important results, a full description of equilibria under the no-acquisition policy as well as precise statements of some additional results mentioned briefly in the main text.

Section B.1 proves the auxiliary results of Section 4.1 and provides some additional ones. In Section B.2, we deal with equilibrium characterization. Section B.3 addresses the effects of the no-acquisition policy. Sections B.4 and B.5 provide the proofs of the statements in Section 6 (Alternative Policies) and the robustness claims of Section 7, respectively. In Section B.6 we introduce the parameterized model capturing consumer surplus effects mentioned in Section 8. Finally, Section B.7 considers a one-dimensional model where firms choose total investment level.

\section{B.1 Auxiliary Results}

This section contains the proofs of the auxiliary results from Section 4.1 and adds a related result for the case where acquisitions are prohibited.

\section{B.1.1 Proof of Lemma 1}

Consider first the commercialization subgame. The entrant commercializes a technology if the payoff from doing so is at least zero. Since $\pi_{E}(L, \ell) \geq \kappa$ by Assumption 2(i) and $\pi(H) \geq \kappa$ by Assumptions 1(i) and 2(ii), the entrant commercializes both technologies. The incumbent commercializes a technology if the payoff of doing so is at least $\pi_{I}(\ell, 0)$. Since $\pi(H)-\kappa \geq \pi_{I}(\ell, 0)$ by Assumption 2(ii), the incumbent always commercializes the $H$ technology. The incumbent commercializes the $L$ technology if and only if $\pi_{I}(L, 0)-$ $\pi_{I}(\ell, 0) \geq \kappa$.

Now consider the acquisitions subgame. There are three possible cases. Either the entrant holds no patent, or he holds the $H$ patent or the $L$ patent. We will examine the three cases in turn. First, suppose that the entrant holds no patent. Then, since the entrant cannot compete without an innovation, the incumbent's profits are the same with or without the acquisition. Thus, the incumbent has no reason to acquire the entrant. Second, suppose the entrant holds a patent on the $H$ technology. Without an acquisition, the entrant commercializes the technology and obtains the payoff $\pi(H)-\kappa$ while the incumbent obtains $\pi_{I}(\ell, H)=0$. With the acquisition, the incumbent commercializes the technology and obtains the payoff $\pi(H)-\kappa$. Thus the total payoffs are equal with or without the acquisition. Since the acquisition (by assumption) only goes through if the total payoffs strictly increase, the incumbent does not acquire the entrant. Third, consider the case when the entrant has a patent for the $L$ technology. If there is no acquisition, 
the entrant commercializes the technology and obtains payoffs $\pi_{E}(L, \ell)-\kappa$, while the incumbent's payoffs are $\pi_{I}(\ell, L)$. If the incumbent acquires the entrant and commercializes the technology, she obtains $\pi_{I}(L, 0)-\kappa$, while without commercialization she obtains $\pi_{I}(\ell, 0)$. Thus she will choose to commercialize only if $\pi_{I}(L, 0)-\kappa \geq \pi_{I}(\ell, 0)$. The incumbent's payoff is $\max \left\{\pi_{I}(L, 0)-\kappa, \pi_{I}(\ell, 0)\right\}$, while the entrant obtains a payoff of zero. Consequently, the acquisition surplus is positive if and only if $\max \left\{\pi_{I}(L, 0)-\kappa, \pi_{I}(\ell, 0)\right\}>$ $\pi_{E}(L, \ell)+\pi_{I}(\ell, L)-\kappa$. We can add $\kappa$ to both sides of the inequality and use Assumption 1 (iv) to show that this inequality indeed holds:

$$
\max \left\{\pi_{I}(L, 0), \pi_{I}(\ell, 0)+\kappa\right\} \geq \max \left\{\pi_{I}(L, 0), \pi_{I}(\ell, 0)\right\}>\pi_{E}(L, \ell)+\pi_{I}(\ell, L)
$$

\section{B.1.2 Characterization of the order of critical projects}

We now first provide the proof of the result on the ordering of critical projects under laissez-faire, before we state and prove a similar result for the no-acquisition policy.

\section{Proof of Lemma 3}

The result will follow immediately from Steps 1 and 2 below.

Step 1: (a) $\theta_{I}^{2}=\theta_{E}^{2}$ and (b) $\theta_{E}^{2}<\theta_{E}^{1}$.

(a) To prove this statement, note that $v_{I}(H)=v_{E}(H)$. Thus

$$
\begin{aligned}
C\left(\theta_{I}^{2}\right) & =\frac{1}{2}\left[p v_{E}(H)+(1-p)\left(v_{I}(L, 0)-v_{I}(\ell, L)\right)\right] \\
& =\frac{1}{2}\left[p v_{E}(H)+(1-p) v_{E}(L, \ell)\right]=C\left(\theta_{E}^{2}\right)
\end{aligned}
$$

(b) Since $C\left(\theta_{E}^{2}\right)<C\left(\theta_{E}^{1}\right)$, part (b) of Step 1 follows immediately.

Step 2: In the killer-acquisition case, only $\theta_{I}^{1}<\theta_{E}^{1}$ is possible.

To see this, note that in the killer-acquisition case, $\max \left\{\pi_{I}(L, 0)-\kappa, \pi_{I}(\ell, 0)\right\}=\pi_{I}(\ell, 0)$ has to hold, so that $v_{I}(L, 0)=v_{I}(\ell, 0)$. Then $\theta_{I}^{1}<\theta_{E}^{1}$ if and only if:

$$
\begin{aligned}
C\left(\theta_{I}^{1}\right) & <C\left(\theta_{E}^{1}\right) \\
p v_{I}(H)+(1-p) v_{I}(L, 0)-v_{I}(\ell, 0) & <p v_{E}(H)+(1-p) v_{E}(L, \ell) \\
-p v_{I}(\ell, 0) & <(1-p) v_{E}(L, \ell),
\end{aligned}
$$

which always holds.

\section{Critical Projects under a No-acquisition Policy}

Lemma B.1. Consider the no-acquisition policy. 
(i) In the killer-acquisition case, the following relations hold:

(a) $\theta_{E}^{2}<\theta_{I}^{2}$; (b) $\theta_{E}^{2}<\theta_{E}^{1}$; (c) $\theta_{I}^{1}<\theta_{E}^{1}$.

(ii) In the genuine-acquisition case, the following relations hold:

(a) $\theta_{E}^{2}<\theta_{I}^{2}$; (b) $\theta_{E}^{2}<\theta_{E}^{1}$.

Proof. Note that (a) and (b) are the same in both the killer- and genuine-acquisition case. We prove them without distinguishing between the cases.

(a): Note that $v_{E}(H)=v_{I}(H) . \theta_{E}^{2}<\theta_{I}^{2}$ will hold if and only if:

$$
\begin{aligned}
C\left(\theta_{E}^{2}\right) & <C\left(\theta_{I}^{2}\right) \\
v_{E}(L, \ell) & <v_{I}(L, 0)-v_{I}(\ell, L) \\
\pi_{E}(L, \ell)-\kappa & <\max \left\{\pi_{I}(\ell, 0), \pi_{I}(L, 0)-\kappa\right\}-\pi_{I}(\ell, L)
\end{aligned}
$$

which is satisfied by Assumption 1(iv).

(b): Since $C\left(\theta_{E}^{2}\right)<C\left(\theta_{E}^{1}\right)$, it follows immediately that $\theta_{E}^{2}<\theta_{E}^{1}$.

For $(c)$, we restrict attention to the killer-acquisition case.

(c): Consider the killer-acquisition case. The claim will hold if and only if

$$
\begin{aligned}
C\left(\theta_{I}^{1}\right) & <C\left(\theta_{E}^{1}\right) \\
p v_{I}(H)+(1-p) v_{I}(L, 0)-v_{I}(\ell, 0) & <p v_{E}(H)+(1-p) v_{E}(L, \ell) \\
-p v_{I}(\ell, 0) & <(1-p) v_{E}(L, \ell)
\end{aligned}
$$

where the equivalence of the last two lines follows from $v_{I}(L, 0)=v_{I}(\ell, 0)$, which always holds in the killer-acquisition case, and Assumption 1(i).

\section{B.2 Equilibrium Characterizations}

In this section, we provide the details on the equilibrium characterization results under laissez-faire and the no-acquisition policy, respectively. In Section B.2.1, we prove the general equilibrium characterization result for each conceivable constellation of critical projects that we already presented in Appendix A. In Section B.2.2, we then state equilibrium characterization results for the no-acquisition policy (Propositions B.1 and B.2). Finally, Section B.2.3 contains the proofs of the equilibrium characterizations Propositions 1, B.1 and B.2, which are straightforward implications of B.2.

\section{B.2.1 General Characterization Result}

We now prove Proposition A.1, the foundation of our equilibrium characterizations (Propositions 1, B.1 and B.2). In the proof, we will require the following immediate implication 
of Lemmas 3 and B.1.

Corollary B.1. Irrespective of policy, the following relations hold:

(i) $\theta_{E}^{1}>\theta_{E}^{2}$

(ii) $\theta_{I}^{2} \geq \theta_{E}^{2}$.

We are now ready to prove Proposition A.1.

Proof. (a) Projects in this interval are (weakly) profitable for the entrant irrespective of the behavior of the incumbent since $\theta \leq \theta_{E}^{2}<\theta_{E}^{1}$ by Corollary B.1(i). Given that the entrant invests, investing is also profitable for the incumbent, as $\theta \leq \theta_{I}^{2}$ by Corollary B.1(ii). Consequently, investment behavior on this interval is consistent with an equilibrium if and only if $r_{E}(\theta)=1$ and $r_{I}(\theta)=1$.

(b) Projects in this interval are never profitable for the entrant irrespective of the behavior of the incumbent since $\theta_{E}^{2}<\theta_{E}^{1}<\theta$ by Corollary B.1(i). As the entrant does not invest, investment is not profitable for the incumbent as $\theta>\theta_{I}^{1}$.

(c) In this interval, it is a unique best response of the incumbent not to invest irrespective of the investment of the entrant. Therefore, using $\theta \leq \theta_{E}^{1}$, it is always a unique best response of the entrant to choose $r_{I}(\theta)=1$.

(d) By now straightforward considerations, there can be no equilibrium where firms choose zero or maximal investment (on a positive measure of projects). Thus, any equilibrium must involve firms choosing strictly interior equilibrium efforts. However, since project payoffs are linear in effort, interior equilibrium efforts can only be sustained when each firm makes the rival exactly indifferent between investing and not investing into a given project. The incumbent is indifferent if and only if

$$
\begin{aligned}
\left(1-r_{E}(\theta)\right) v_{I}(\ell, 0)+r_{E}(\theta)(1-p) v_{I}(\ell, L) & = \\
-C(\theta)+\left(1-r_{E}(\theta)\right) & {\left[p v_{I}(H)+(1-p) v_{I}(L, 0)\right] } \\
& +r_{E}(\theta) \frac{1}{2}\left[p v_{I}(H)+(1-p)\left(v_{I}(L, 0)+v_{I}(\ell, L)\right)\right]
\end{aligned}
$$

where the LHS represents the incumbent's project payoff from investing 0 and the RHS represents the incumbent's project payoff from investing 1 .

Using the definitions of $C\left(\theta_{I}^{1}\right)$ and $C\left(\theta_{I}^{2}\right)$ and solving for $r_{E}(\theta)$, we arrive at $r_{E}(\theta)=$ $\frac{C(\theta)-C\left(\theta_{I}^{1}\right)}{C\left(\theta_{I}^{2}\right)-C\left(\theta_{I}^{1}\right)}$, which is the unique solution to the above equation. We can proceed analogously to arrive at $r_{I}(\theta)=\frac{C\left(\theta_{E}^{1}\right)-C(\theta)}{C\left(\theta_{E}^{1}\right)-C\left(\theta_{E}^{2}\right)}$. Moreover, these investment levels are feasible, since they are between 0 and 1 for every $\theta \in\left(\max \left\{\theta_{I}^{1}, \theta_{E}^{2}\right\}, \min \left\{\theta_{I}^{2}, \theta_{E}^{1}\right\}\right]$. Thus, effort levels $r_{E}$ and $r_{I}$ for each project $\theta$ in this interval are consistent with equilibrium behavior if and only if they are defined as in (d). 
(e) It is simple to show that the strategies delineated in Definition 1 (i) and (ii) constitute equilibrium behavior and that no other equilibrium with minimal or maximal effort choices exists. Proceeding as in (d), we find that the strategy in Definition 1 (iii) is also consistent with equilibrium behavior for $\theta$ in this interval, and there is no other strategy with interior effort levels for which this is the case.

(f) In (a)-(e), we have shown that, if $\theta$ lies in the given interval for each of the cases, we arrive at the respective equilibrium behavior for project $\theta$.

We now show that in all remaining cases one of the following must hold:

(i) $\theta \in\left(\theta_{E}^{2}, \min \left\{\theta_{I}^{1}, \theta_{I}^{2}\right\}\right]$ and $\min \left\{\theta_{I}^{1}, \theta_{I}^{2}\right\}<\theta_{E}^{1}$

(ii) $\theta \in\left(\max \left\{\theta_{I}^{2}, \theta_{E}^{1}\right\}, \theta_{I}^{1}\right]$

(iii) $\theta \in\left(\theta_{E}^{2}, \theta_{I}^{1}\right]$ and $\min \left\{\theta_{I}^{1}, \theta_{I}^{2}\right\} \geq \theta_{E}^{1}$

All equilibria satisfy $(a)$ and $(b)$, but which ones of the remaining cases apply in the interval $\left(\theta_{E}^{2}, \max \left\{\theta_{I}^{1}, \theta_{E}^{1}\right\}\right.$ depends on the exact order of critical projects. We will thus consider each case $(c)-(f)$ in turn and show that, if there are still intervals not covered, they fall into at least one of the listed cases:

Assuming case $(c)$ occurs, we need to characterize the possible constellations in the interval $\left(\theta_{E}^{2}, \max \left\{\theta_{I}^{2}, \theta_{I}^{1}\right\}\right] . \quad\left(\min \left\{\theta_{I}^{2}, \theta_{I}^{1}\right\}, \max \left\{\theta_{I}^{2}, \theta_{I}^{1}\right\}\right]$ corresponds to case $(d)$ if $\theta_{I}^{1} \leq \theta_{I}^{2}$ and to case $(e)$ if $\theta_{I}^{1}>\theta_{I}^{2}$. Thus, we are left with the interval $\left(\theta_{E}^{2}, \min \left\{\theta_{I}^{1}, \theta_{I}^{2}\right\}\right]$, which is case $(i)$ above.

Assuming case $(d)$ occurs, we need to characterize the possible constellations in the intervals $\left(\theta_{E}^{2}, \max \left\{\theta_{I}^{1}, \theta_{E}^{2}\right\}\right]$ and $\left(\min \left\{\theta_{E}^{1}, \theta_{I}^{2}\right\}, \theta_{E}^{1}\right]$. Since $\left(\min \left\{\theta_{E}^{1}, \theta_{I}^{2}\right\}, \theta_{E}^{1}\right]$ only has positive measure if $\theta_{I}^{2}<\theta_{E}^{1}$, the interval falls into case $(c) . \quad\left(\theta_{E}^{2}, \max \left\{\theta_{I}^{1}, \theta_{E}^{2}\right\}\right]$ only has positive measure if $\theta_{E}^{2}<\theta_{I}^{1}$, and then the interval corresponds to case $(i)$ above.

Assuming case $(e)$ occurs, we need to characterize the possible constellations in the intervals $\left(\theta_{E}^{2}, \theta_{I}^{2}\right]$ and $\left(\min \left\{\theta_{I}^{1}, \theta_{E}^{1}\right\},\left(\max \left\{\theta_{I}^{1}, \theta_{E}^{1}\right\}\right]\right.$. For the second interval, if $\theta_{I}^{1}<\theta_{E}^{1}$, we are in case $(c)$ and if $\theta_{I}^{1} \geq \theta_{E}^{1}$, we are in case $(i i)$ above. $\left(\theta_{E}^{2}, \theta_{I}^{2}\right]$ corresponds to case $(i)$ above.

Cases $(c),(d)$ and $(e)$ all require $\min \left\{\theta_{I}^{1}, \theta_{I}^{2}\right\}<\theta_{E}^{1}$. Assuming that $\min \left\{\theta_{I}^{1}, \theta_{I}^{2}\right\} \geq \theta_{E}^{1}$ implies that neither $(c),(d)$ or $(e)$ occurs. Case $(i i i)$ above therefore covers the whole interval $\left(\theta_{E}^{2}, \theta_{I}^{1}\right]$. Moreover, if $\min \left\{\theta_{I}^{1}, \theta_{I}^{2}\right\}<\theta_{E}^{1}$, at least one of the three cases, $(c),(d)$ or $(e)$, occurs and thus there are no cases left to consider.

Having established that we identified the remaining cases, we can use arguments that are standard by now to show that efforts in each of those cases are consistent with equilibrium behavior if and only if $r_{E}(\theta)=0$ and $r_{I}(\theta)=1$. 


\section{B.2.2 Equilibria under a No-acquisition Policy}

We now state equilibrium characterizations for the no-acquisition policy (first for the killeracquisition case, then for the genuine-acquisition case). The proofs are in Section B.2.3.

Proposition B.1 (Killer acquisition). Consider the killer-acquisition case under a noacquisition policy. In any equilibrium under laissez-faire,

$$
\begin{aligned}
& \text { (A) } r_{E}(\theta)=1 \text { and } r_{I}(\theta)=1 \text { for } \theta \in\left[0, \theta_{E}^{2}\right] \\
& \text { (B) } r_{E}(\theta)=0 \text { and } r_{I}(\theta)=0 \text { for } \theta \in\left(\theta_{E}^{1}, 1\right) .
\end{aligned}
$$

(a) If $\theta_{I}^{2} \geq \theta_{I}^{1}$, then $\max \left\{\theta_{I}^{1}, \theta_{E}^{2}\right\} \leq \min \left\{\theta_{I}^{2}, \theta_{E}^{1}\right\}$. There is a unique equilibrium. Functions $r_{I}$ and $r_{E}$ constitute an equilibrium if and only if, in addition to $(A)$ and $(B)$, the following conditions hold:

$$
\begin{aligned}
& \text { (i) } r_{E}(\theta)=0 \text { and } r_{I}(\theta)=1 \text { for } \theta \in\left(\theta_{E}^{2}, \max \left\{\theta_{I}^{1}, \theta_{E}^{2}\right\}\right] \\
& \text { (ii) } r_{E}(\theta)=\frac{C(\theta)-C\left(\theta_{I}^{1}\right)}{C\left(\theta_{I}^{2}\right)-C\left(\theta_{I}^{1}\right)} \text { and } r_{I}(\theta)=\frac{C\left(\theta_{E}^{1}\right)-C(\theta)}{C\left(\theta_{E}^{1}\right)-C\left(\theta_{E}^{2}\right)}, \\
& \text { for } \theta \in\left(\max \left\{\theta_{I}^{1}, \theta_{E}^{2}\right\}, \min \left\{\theta_{I}^{2}, \theta_{E}^{1}\right\}\right], \\
& \text { (iii) } r_{E}(\theta)=1 \text { and } r_{I}(\theta)=0 \text { for } \theta \in\left(\min \left\{\theta_{I}^{2}, \theta_{E}^{1}\right\}, \theta_{E}^{1}\right],
\end{aligned}
$$

(b) If $\theta_{I}^{2}<\theta_{I}^{1}$ then $\theta_{E}^{2}<\theta_{I}^{2}<\theta_{I}^{1}<\theta_{E}^{1}$. The equilibrium is not unique. Functions $r_{I}$ and $r_{E}$ constitute an equilibrium if and only if, in addition to $(A)$ and $(B)$, the following conditions hold:

(i) $r_{E}(\theta)=0$ and $r_{I}(\theta)=1$ for $\theta \in\left(\theta_{E}^{2}, \theta_{I}^{2}\right]$

(ii) $r_{E}(\theta)=1$ and $r_{I}(\theta)=0$ for $\theta \in\left(\theta_{I}^{1}, \theta_{E}^{1}\right]$,

(iii) Firms use an anti-coordination profile for $\theta \in\left(\theta_{I}^{2}, \theta_{I}^{1}\right]$.

Proposition B.2 (Genuine acquisition). Consider the genuine-acquisition case under a no-acquisition policy. In any equilibrium,

$$
\begin{aligned}
& \text { (A) } r_{E}(\theta)=1 \text { and } r_{I}(\theta)=1 \text { for } \theta \in\left[0, \theta_{E}^{2}\right], \\
& \text { (B) } r_{E}(\theta)=0 \text { and } r_{I}(\theta)=0 \text { for } \theta \in\left(\max \left\{\theta_{I}^{1}, \theta_{E}^{1}\right\}, 1\right) .
\end{aligned}
$$

(a) If $\theta_{I}^{2} \geq \theta_{I}^{1}$, then $\max \left\{\theta_{I}^{1}, \theta_{E}^{2}\right\} \leq \min \left\{\theta_{I}^{2}, \theta_{E}^{1}\right\}$ and there is a unique equilibrium. Functions $r_{I}$ and $r_{E}$ constitute an equilibrium if and only if, in addition to $(A)$ and $(B)$, the following conditions hold:

(i) $r_{E}(\theta)=0$ and $r_{I}(\theta)=1$ for $\theta \in\left(\theta_{E}^{2}, \max \left\{\theta_{I}^{1}, \theta_{E}^{2}\right\}\right]$

(ii) $r_{E}(\theta)=\frac{C(\theta)-C\left(\theta_{I}^{1}\right)}{C\left(\theta_{I}^{2}\right)-C\left(\theta_{I}^{1}\right)}$ and $r_{I}(\theta)=\frac{C\left(\theta_{E}^{1}\right)-C(\theta)}{C\left(\theta_{E}^{1}\right)-C\left(\theta_{E}^{2}\right)}$

for $\theta \in\left(\max \left\{\theta_{I}^{1}, \theta_{E}^{2}\right\}, \min \left\{\theta_{I}^{2}, \theta_{E}^{1}\right\}\right]$, 
(iii) $r_{E}(\theta)=1$ and $r_{I}(\theta)=0$ for $\theta \in\left(\min \left\{\theta_{I}^{2}, \theta_{E}^{1}\right\}, \theta_{E}^{1}\right]$.

(b) If $\theta_{I}^{2}<\theta_{I}^{1}<\theta_{E}^{1}$, then $\theta_{E}^{2}<\theta_{I}^{2}<\theta_{I}^{1}<\theta_{E}^{1}$. The equilibrium is not unique. Functions $r_{I}$ and $r_{E}$ constitute an equilibrium if and only if, in addition to $(A)$ and $(B)$, the following conditions hold:

(i) $r_{E}(\theta)=0$ and $r_{I}(\theta)=1$ for $\theta \in\left(\theta_{E}^{2}, \theta_{I}^{2}\right]$

(ii) $r_{E}(\theta)=1$ and $r_{I}(\theta)=0$ for $\theta \in\left(\theta_{I}^{1}, \theta_{E}^{1}\right]$,

(iii) Firms use an anti-coordination profile for $\theta \in\left(\theta_{I}^{2}, \theta_{I}^{1}\right]$.

(c) If $\theta_{I}^{2}<\theta_{E}^{1} \leq \theta_{I}^{1}$, then $\theta_{E}^{2}<\theta_{I}^{2}<\theta_{E}^{1} \leq \theta_{I}^{1}$. The equilibrium is not unique. Functions $r_{I}$ and $r_{E}$ constitute an equilibrium if and only if, in addition to $(A)$ and $(B)$, the following conditions hold:

(i) $r_{E}(\theta)=0$ and $r_{I}(\theta)=1$ for $\theta \in\left(\theta_{E}^{2}, \theta_{I}^{2}\right]$

(ii) $r_{E}(\theta)=0$ and $r_{I}(\theta)=1$ for $\theta \in\left(\theta_{E}^{1}, \theta_{I}^{1}\right]$,

(iii) Firms use an anti-coordination profile for $\theta \in\left(\theta_{I}^{2}, \theta_{E}^{1}\right]$.

(d) If $\theta_{E}^{1} \leq \min \left\{\theta_{I}^{2}, \theta_{I}^{1}\right\}$, then $\theta_{E}^{2}<\theta_{E}^{1} \leq \min \left\{\theta_{I}^{1}, \theta_{I}^{2}\right\}$. The equilibrium is unique. Functions $r_{I}$ and $r_{E}$ constitute an equilibrium if and only if, in addition to $(A)$ and $(B)$, $r_{E}(\theta)=0$ and $r_{I}(\theta)=1$, for $\theta \in\left(\theta_{E}^{2}, \theta_{I}^{1}\right]$.

\section{B.2.3 Proof of Equilibrium Characterizations}

Proof of Proposition 1 According to Lemma 3, in the laissez-faire regime one of the following constellations applies:
(i) $\theta_{I}^{1} \leq \theta^{2}<\theta_{E}^{1}$
(ii) $\theta^{2}<\theta_{I}^{1}<\theta_{E}^{1}$
(iii) $\theta^{2}<\theta_{E}^{1} \leq \theta_{I}^{1}$.

Applying Proposition A.1 to each constellation gives the result.

Proof of Proposition B.1 According to Lemma B.1(i), under the no-acquisition policy in the killer-acquisition case, one of the following five constellations applies:
(i) $\theta_{I}^{1} \leq \theta_{E}^{2}<\theta_{I}^{2} \leq \theta_{E}^{1}$
(ii) $\theta_{I}^{1} \leq \theta_{E}^{2}<\theta_{E}^{1}<\theta_{I}^{2}$
(iii) $\theta_{E}^{2}<\theta_{I}^{1} \leq \theta_{I}^{2} \leq \theta_{E}^{1}$
(iv) $\theta_{E}^{2}<\theta_{I}^{1}<\theta_{E}^{1}<\theta_{I}^{2}$
(v) $\theta_{E}^{2}<\theta_{I}^{2}<\theta_{I}^{1}<\theta_{E}^{1}$.

Applying Proposition A.1 to each constellation gives the result. 
Proof of Proposition B.2 According to Lemma B.1(ii), under the no-acquisitions policy in the genuine-acquisition case, one of the following constellations applies:
(i) $\theta_{I}^{1} \leq \theta_{E}^{2}<\theta_{I}^{2} \leq \theta_{E}^{1}$
(ii) $\theta_{I}^{1} \leq \theta_{E}^{2}<\theta_{E}^{1}<\theta_{I}^{2}$
(iii) $\theta_{E}^{2}<\theta_{I}^{1} \leq \theta_{I}^{2} \leq \theta_{E}^{1}$
(iv) $\theta_{E}^{2}<\theta_{I}^{1} \leq \theta_{E}^{1}<\theta_{I}^{2}$
(v) $\theta_{E}^{2}<\theta_{I}^{2}<\theta_{I}^{1} \leq \theta_{E}^{1}$
(vi) $\theta_{E}^{2}<\theta_{E}^{1}<\theta_{I}^{1} \leq \theta_{I}^{2}$
(vii) $\theta_{E}^{2}<\theta_{E}^{1} \leq \theta_{I}^{2}<\theta_{I}^{1}$
(viii) $\theta_{E}^{2}<\theta_{I}^{2}<\theta_{E}^{1}<\theta_{I}^{1}$.

Applying Proposition A.1 to each constellation gives the result.

\section{B.3 The Effects of Prohibiting Acquisitions}

This section contains details on the effects of prohibiting acquisitions. In Section B.3.1, we prove Proposition 2. Section B.3.2 gives conditions under which the innovation effect of prohibiting acquisitions is zero. Section B.3.3 contains the proof of Proposition 3. In Section B.3.4, we evaluate the effect of prohibiting acquisitions on duplication of $R \& D$ investment, as discussed towards the end of Section 5 .

\section{B.3.1 Proof of Proposition 2}

Denote the equilibrium strategies under laissez-faire and the no-acquisition policy as $\left(r_{I}^{A}, r_{E}^{A}\right)$ and $\left(r_{I}^{N}, r_{E}^{N}\right)$, respectively. The result follows from Steps 1-5.

Step 1: $\mathcal{V}^{A}=\max \left\{\theta_{E}^{1}(A), \theta_{I}^{1}(A)\right\}$ and $\mathcal{V}^{N}=\max \left\{\theta_{E}^{1}(N), \theta_{I}^{1}(N)\right\}$.

The first claim holds because, by Proposition $1, r_{I}^{A}(\theta)+r_{E}^{A}(\theta)=0$ if and only if $\theta \in$ $\left(\max \left\{\theta_{E}^{1}(A), \theta_{I}^{1}(A)\right\}, 1\right)$. Hence, $\mathcal{V}^{A}=\max \left\{\theta_{E}^{1}(A), \theta_{I}^{1}(A)\right\}$. The second claim holds because, by Propositions B.1 and B.2, $r_{I}^{N}(\theta)+r_{E}^{N}(\theta)=0$ if and only if $\theta \in\left(\max \left\{\theta_{E}^{1}(N), \theta_{I}^{1}(N)\right\}, 1\right)$.

Step 2: $\theta_{I}^{1}(A)=\theta_{I}^{1}(N)$.

To show this, it is sufficient that $C\left(\theta_{I}^{1}(A)\right)=C\left(\theta_{I}^{1}(N)\right)$, or equivalently

$$
p v_{I}^{A}(H)+(1-p) v_{I}^{A}(L, 0)-v_{I}^{A}(\ell, 0)=p v_{I}^{N}(H)+(1-p) v_{I}^{N}(L, 0)-v_{I}^{N}(\ell, 0) .
$$

This holds since $v_{I}^{A}(t, 0)=v_{I}^{N}(t, 0)$ for all $t \in\{\ell, L, H\}$.

Step 3: $\theta_{E}^{1}(N)<\theta_{E}^{1}(A)$.

To show this, it is sufficient that $C\left(\theta_{E}^{1}(N)\right)<C\left(\theta_{E}^{1}(A)\right)$ The claim requires that

$$
p v_{E}^{N}(H)+(1-p) v_{E}^{N}(L, \ell)<p v_{E}^{A}(H)+(1-p) v_{E}^{A}(L, \ell) .
$$

This holds because

$$
\begin{aligned}
& p(\pi(H)-\kappa)+(1-p)\left(\pi_{E}(L, \ell)-\kappa\right)<p(\pi(H)-\kappa)+(1-p) v_{E}^{A}(L, \ell) \\
& \pi_{E}(L, \ell)-\kappa<\beta\left(\max \left\{\pi_{I}(L, 0)-\kappa, \pi_{I}(\ell, 0)\right\}-\pi_{I}(\ell, L)\right)+(1-\beta)\left(\pi_{E}(L, \ell)-\kappa\right)
\end{aligned}
$$




$$
\pi_{E}(L, \ell)-\kappa<\max \left\{\pi_{I}(L, 0)-\kappa, \pi_{I}(\ell, 0)\right\}-\pi_{I}(\ell, L)
$$

where simple algebra leads to the last inequality, which holds by Assumption 1(iv).

Step 4: If $\theta_{E}^{1}(A)>\theta_{I}^{1}(A)$, then $\mathcal{V}^{A}>\mathcal{V}^{N}$ and if $\left(r_{I}^{A}, r_{E}^{A}\right)$ is a simple equilibrium then $\mathcal{P}\left(r_{I}^{A}, r_{E}^{A}\right)>\mathcal{P}\left(r_{I}^{N}, r_{E}^{N}\right)$.

Since $\theta_{E}^{1}(A)>\theta_{E}^{1}(N)$ by Step 3 and $\theta_{I}^{1}(A)=\theta_{I}^{1}(N)$ by Step 2, we obtain $\theta_{E}^{1}(A)>$ $\max \left\{\theta_{E}^{1}(N), \theta_{I}^{1}(N)\right\}$. Hence, $\mathcal{V}^{A}>\mathcal{V}^{N}$. Since $\mathcal{P}\left(r_{I}, r_{E}\right) \leq \mathcal{V}\left(r_{I}, r_{E}\right)$ for any $\left(r_{I}, r_{E}\right)$ and $\mathcal{P}\left(r_{I}, r_{E}\right)=\mathcal{V}\left(r_{I}, r_{E}\right)$ for simple equilibria, then also $\mathcal{P}\left(r_{I}^{A}, r_{E}^{A}\right)>\mathcal{P}\left(r_{I}^{N}, r_{E}^{N}\right)$ if $\left(r_{I}^{A}, r_{E}^{A}\right)$ is a simple equilibrium.

Step 5: If $\theta_{E}^{1}(A) \leq \theta_{I}^{1}(A)$, then $\mathcal{V}^{A}=\mathcal{V}^{N}$ and if $\left(r_{I}^{A}, r_{E}^{A}\right)$ is a simple equilibrium then $\mathcal{P}\left(r_{I}^{A}, r_{E}^{A}\right) \geq \mathcal{P}\left(r_{I}^{N}, r_{E}^{N}\right)$.

If $\theta_{E}^{1}(A) \leq \theta_{I}^{1}(A)$, then by Steps 2 and $3, \theta_{E}^{1}(N)<\theta_{I}^{1}(N)$. Then $\mathcal{V}^{A}=\theta_{I}^{1}(A)=\theta_{I}^{1}(N)=$ $\mathcal{V}^{N}$. Since $\mathcal{P}\left(r_{I}, r_{E}\right) \leq \mathcal{V}\left(r_{I}, r_{E}\right)$ for any $\left(r_{I}, r_{E}\right)$ and $\mathcal{P}\left(r_{I}, r_{E}\right)=\mathcal{V}\left(r_{I}, r_{E}\right)$ for simple equilibria, then also $\mathcal{P}\left(r_{I}^{A}, r_{E}^{A}\right) \geq \mathcal{P}\left(r_{I}^{N}, r_{E}^{N}\right)$.

\section{B.3.2 Conditions for the Absence of an Innovation Effect}

We now present and prove the result mentioned in Section 5.2 which gives conditions under which the innovation effect is zero.

Proposition B.3. (i) Suppose $\Pi=\left(\pi_{I}(L, 0), \pi_{I}(\ell, 0), \pi_{I}(\ell, L), \pi_{E}(L, \ell), \pi(H)\right)$ satisfies Assumption 1. Then there exists a vector $(\kappa, p, \beta) \in \mathbb{R}^{+} \times[0,1] \times[0,1]$ such that $(a)(\Pi, \kappa)$ is consistent with Assumption 2 and (b) the innovation effect is zero if and only if the following condition holds:

$$
\pi_{I}(L, 0)-\pi_{I}(\ell, 0) \geq \pi_{E}(L, \ell)
$$

(ii) If (1) holds and $\pi_{I}(\ell, L)<\pi_{I}(\ell, 0)$, there exists $a \widehat{p} \in[0,1)$ and $\widehat{\beta} \in[0,1)$ and a weakly decreasing function $P(\beta):[0, \widehat{\beta}] \rightarrow[0,1]$ with $P(0)=\widehat{p}$ and $P(\widehat{\beta})=0$ such that the innovation effect is zero for any $(\beta, \kappa, p)$ such that Assumption 2 holds and

$$
\begin{aligned}
\pi_{I}(L, 0)-\pi_{I}(\ell, 0) & \geq \kappa \\
0 & \leq \beta \leq \widehat{\beta} \\
0 & \leq p \leq P(\beta)
\end{aligned}
$$

Proof. We will first show that the requirements of consistency with Assumption 2 and absence of an innovation effect are easiest to fulfill if $(\beta, \kappa, p)=(0,0,0)$. In other words, if, for fixed vector $\Pi$ the requirements hold for any $(\kappa, p, \beta) \in \mathbb{R}^{+} \times[0,1] \times[0,1]$, they hold 
for $(\kappa, p, \beta)=(0,0,0)$. To see this, first note that Assumption 2 requires that

$$
\begin{aligned}
\pi_{E}(L, \ell) & \geq \kappa \text { and } \\
\pi(H)-\pi_{I}(\ell, 0) & \geq \kappa,
\end{aligned}
$$

so that it is easiest to satisfy for $\kappa=0$. Next, the condition under which there is no innovation effect is that there is commercialization,

$$
\pi_{I}(L, 0)-\pi_{I}(\ell, 0) \geq \kappa
$$

and

$$
(1-p) v_{I}(L, 0)-v_{I}(\ell, 0) \geq(1-p) v_{E}(L, \ell)
$$

Substituting expressions from Lemma 2 and rearranging, (5) can be expressed as

$$
(1-p)\left[(1-\beta)\left(\pi_{I}(L, 0)-\pi_{E}(L, \ell)\right)+\beta \pi_{I}(\ell, L)\right] \geq \pi_{I}(\ell, 0) .
$$

To fulfil the commercialization condition (4) at least for $\kappa=0, \Pi$ must satisfy $\pi_{I}(L, 0) \geq$ $\pi_{I}(\ell, 0)$. Then Assumption 1(iv) implies

$$
\pi_{I}(L, 0)-\pi_{E}(L, \ell)-\pi_{I}(\ell, L)>0 .
$$

Thus, the LHS in (6) is strictly decreasing in $\beta$ for $p<1$. (7) implies that $\pi_{I}(L, 0)-$ $\pi_{E}(L, \ell)>0$. By Assumption $1(\mathrm{i}), \pi_{I}(\ell, L) \geq 0$. Therefore, the square bracket in $(6)$ is positive and the LHS is decreasing in $p$ as long as $\beta<1$. Thus, (6) is easiest to fulfill if $p=0$ and $\beta=0$. All told, therefore, if $(3),(6)$ and (7) hold for any $(\kappa, p, \beta) \in \mathbb{R}^{+} \times[0,1] \times[0,1]$, they hold for $(\kappa, p, \beta)=(0,0,0)$.

Thus, for there to be a zero innovation effect for any $(\kappa, p, \beta) \in \mathbb{R}^{+} \times[0,1] \times[0,1]$ such that Assumption 2 hold is that $\Pi$ satisfies the following four conditions:

$$
\begin{aligned}
\pi_{I}(L, 0)-\pi_{I}(\ell, 0) & \geq \pi_{E}(L, \ell) \\
\pi_{I}(L, 0)-\pi_{I}(\ell, 0) & \geq 0 \\
\pi_{E}(L, \ell) & \geq 0 \\
\pi(H)-\pi_{I}(\ell, 0) & \geq 0
\end{aligned}
$$

In particular, therefore (1) holds. This proves the "only if"-part of (i).

As to the "if"-part, note that $\pi_{E}(L, \ell) \geq 0$ by Assumption 1(i). Thus, the first three conditions of $(8)$ reduce to $\pi_{I}(L, 0)-\pi_{I}(\ell, 0) \geq \pi_{E}(L, \ell)$. Assumption 1 further implies 
that this condition is at least as restrictive as $\pi(H)-\pi_{I}(\ell, 0) \geq 0$. Hence, the four conditions in (8) are fulfilled if (1) holds. Under these conditions, $\Pi$ and $(\kappa, \beta, p)=$ $(0,0,0)$ jointly satisfy all requirements for the absence of an innovation effect.

(ii) Part (i) has already shown that (4) and (6) both hold for $\Pi$ and $(\kappa, p, \beta)=(0,0,0)$ if $\Pi$ satisfies (1). Next, (6) is violated for $(p, \beta)=(0,1)$ : It simplifies to $\pi_{I}(\ell, L) \geq \pi_{I}(\ell, 0)$. Similarly, (6) is violated for $(p, \beta)=(1,0)$ : It reduces to $0 \geq \pi_{I}(\ell, 0)$, which is inconsistent with the positivity of monopoly profits (Assumption 1(i)).

Finally, by Assumption 1, the LHS of (6) is decreasing and continuous in $\beta$ and in $p$. Thus, by the intermediate value theorem there exist $\widehat{p}$ and $\widehat{\beta}$ such that (6) holds with equality for $(\widehat{p}, 0)$ and $(0, \widehat{\beta})$ and with inequality for $(p, 0)$ with $p<\widehat{p}$ and for $(0, \beta)$ with $\beta<\widehat{\beta}$. Thus, the statement holds for $\beta=0$ and $\beta=\widehat{\beta}$ with $P(0)=\widehat{p}$ and $P(\widehat{\beta})=0$. The fact that the LHS of (6) is weakly decreasing then leads to the result for $\beta \in(0, \widehat{\beta})$.

Intuitively, the necessary condition (1) in (i) for the innovation effect to be zero is that the innovation would increase incumbent monopoly profits by a large amount, while, under duopoly competition, the entrant's profits would be relatively low (competition is either intense or biased against the entrant). Once this condition on product market profits holds, the innovation effect will be zero according to (ii) if $\kappa, p$ and $\beta$ are sufficiently low. Thus, if these conditions hold together, then one can take decisions entirely based on the competition effect.

\section{B.3.3 Proof of Proposition 3}

Proposition 2(i) implies $\Delta_{\mathcal{V}}=\mathcal{V}^{A}-\mathcal{V}^{N}=\max \left\{\theta_{E}^{1}(A), \theta_{I}^{1}(A)\right\}-\max \left\{\theta_{E}^{1}(N), \theta_{I}^{1}(N)\right\} \geq 0$, where $\theta_{I}^{1}(A)=\theta_{I}^{1}(N)=\theta_{I}^{1}$ and $\theta_{E}^{1}(A)>\theta_{E}^{1}(N)$. We will analyze the change of $\Delta_{\mathcal{V}}$ as a result of a change in $\beta, \pi_{I}(\ell, L)$ and $\pi_{E}(L, \ell)$ for all orderings of $\theta_{I}^{1}, \theta_{E}^{1}(A)$ and $\theta_{E}^{1}(N)$.

This gives us five cases, which we analyze below. The proposition aggregates the effects in these five cases.

Case 1: If $\theta_{I}^{1}<\theta_{E}^{1}(N)<\theta_{E}^{1}(A)$, then $\Delta_{\mathcal{V}}=\theta_{E}^{1}(A)-\theta_{E}^{1}(N)$. Applying the inverse function theorem, we obtain:

(a) $\partial \Delta_{\mathcal{V}} / \partial \beta>0$ is equivalent with

$$
\frac{\partial\left(\theta_{E}^{1}(A)-\theta_{E}^{1}(N)\right)}{\partial \beta}=\frac{(1-p)\left(\max \left\{\pi_{I}(L, 0)-\kappa, \pi_{I}(\ell, 0)\right\}-\pi_{I}(\ell, L)-\pi_{E}(L, \ell)+\kappa\right)}{C^{\prime}\left(\theta_{E}^{1}(A)\right)}>0
$$

which follows from Assumption 1(iv). 
(b) $\partial \Delta_{\mathcal{V}} / \partial \pi_{I}(\ell, L)<0 \quad$ is equivalent with

$$
\frac{\partial\left(\theta_{E}^{1}(A)-\theta_{E}^{1}(N)\right)}{\partial \pi_{I}(\ell, L)}=\frac{-(1-p) \beta}{C^{\prime}\left(\theta_{E}^{1}(A)\right)}<0
$$

(c) $\partial \Delta_{\mathcal{V}} / \partial \pi_{E}(L, \ell)<0$ is equivalent with

$$
\begin{aligned}
\frac{\partial\left(\theta_{E}^{1}(A)-\theta_{E}^{1}(N)\right)}{\partial \pi_{E}(L, \ell)} & =\frac{(1-p)(1-\beta)}{C^{\prime}\left(\theta_{E}^{1}(A)\right)}-\frac{(1-p)}{C^{\prime}\left(\theta_{E}^{1}(N)\right)}<0 \\
& \Leftrightarrow(1-\beta)<\frac{C^{\prime}\left(\theta_{E}^{1}(A)\right)}{C^{\prime}\left(\theta_{E}^{1}(N)\right)}
\end{aligned}
$$

where the inequality follows from the convexity of $C$.

Case 2: If $\theta_{E}^{1}(N)<\theta_{I}^{1}<\theta_{E}^{1}(A)$, then $\Delta_{\mathcal{V}}=\theta_{E}^{1}(A)-\theta_{I}^{1}$. Again applying the inverse function theorem:

(a) $\partial \Delta_{\mathcal{V}} / \partial \beta>0$ is equivalent with

$$
\frac{\partial\left(\theta_{E}^{1}(A)-\theta_{I}^{1}\right)}{\partial \beta}=\frac{(1-p)\left(\max \left\{\pi_{I}(L, 0)-\kappa, \pi_{I}(\ell, 0)\right\}-\pi_{I}(\ell, L)-\pi_{E}(L, \ell)+\kappa\right)}{C^{\prime}\left(\theta_{E}^{1}(A)\right)}>0
$$

which follows from Assumption 1(iv).

(b) $\partial \Delta_{\mathcal{V}} / \partial \pi_{I}(\ell, L)<0 \quad$ is equivalent with

$$
\frac{\partial\left(\theta_{E}^{1}(A)-\theta_{I}^{1}\right)}{\partial \pi_{I}(\ell, L)}=\frac{-(1-p) \beta}{C^{\prime}\left(\theta_{E}^{1}(A)\right)}<0
$$

(c) $\partial \Delta_{\mathcal{V}} / \partial \pi_{E}(L, \ell)>0$ is equivalent with

$$
\frac{\partial\left(\theta_{E}^{1}(A)-\theta_{I}^{1}\right)}{\partial \pi_{E}(L, \ell)}=\frac{(1-p)(1-\beta)}{C^{\prime}\left(\theta_{E}^{1}(A)\right)}>0 .
$$

Case 3: If $\theta_{E}^{1}(N)<\theta_{E}^{1}(A)<\theta_{I}^{1}, \Delta_{\mathcal{V}}=0$ and $\partial \Delta_{\mathcal{V}} / \partial x=0$ for $x \in\left\{\beta, \pi_{I}(\ell, L), \pi_{E}(L, \ell)\right\}$. Case 4: If $\theta_{E}^{1}(N)=\theta_{I}^{1}<\theta_{E}^{1}(A)$, then $\Delta_{\mathcal{V}}=\theta_{E}^{1}(A)-\max \left\{\theta_{I}^{1}, \theta_{E}^{1}(N)\right\}$. Provided that the derivative exists, the effect on variety is

$$
\frac{\partial\left(\theta_{E}^{1}(A)-\max \left\{\theta_{I}^{1}, \theta_{E}^{1}(N)\right\}\right)}{\partial x}
$$

Note that $\partial \theta_{I}^{1} / \partial x=0$ and $\partial \theta_{E}^{1}(N) / \partial x=0$ for $x \in\left\{\beta, \pi_{I}(\ell, L)\right\}$, which implies that the derivative exists and $\partial \max \left\{\theta_{I}^{1}, \theta_{E}^{1}(N)\right\} / \partial x=0$. Therefore, $\partial \Delta_{\mathcal{V}} / \partial \beta=\partial \theta_{E}^{1}(A) / \partial \beta>0$ and $\partial \Delta_{\mathcal{V}} / \partial \pi_{I}(\ell, L)=\partial \theta_{E}^{1}(A) / \partial \pi_{I}(\ell, L)<0$. 
Case 5: If $\theta_{E}^{1}(N)<\theta_{I}^{1}=\theta_{E}^{1}(A)$, then $\Delta_{\mathcal{V}}=\max \left\{\theta_{I}^{1}, \theta_{E}^{1}(A)\right\}-\theta_{I}^{1}$. Since $\partial \theta_{I}^{1} / \partial x=0$ for $x \in\left\{\beta, \pi_{I}(\ell, L), \pi_{E}(L, \ell)\right\}$, the effect on variety is equal to the effect on $\max \left\{\theta_{I}^{1}, \theta_{E}^{1}(A)\right\}$.

First, consider the effect of $\beta$. Note that $\partial \theta_{E}^{1}(A) / \partial \beta>0$ and fix $\beta_{0}$ such that $\theta_{I}^{1}\left(\beta_{0}\right)=$ $\theta_{E}^{1}\left(A, \beta_{0}\right)$. Then for all $\beta^{\prime}$ and $\beta^{\prime \prime}$ such that $\beta^{\prime}<\beta_{0}<\beta^{\prime \prime}$, we have $\theta_{I}^{1}\left(\beta^{\prime}\right)>\theta_{E}^{1}\left(A, \beta^{\prime}\right)$ and $\theta_{I}^{1}\left(\beta^{\prime \prime}\right)<\theta_{E}^{1}\left(A, \beta^{\prime \prime}\right)$. Denoting with $\partial_{-}$and $\partial_{+}$the left and the right derivative (at $\left.\beta_{0}\right)$ respectively, the argument above implies

$$
\frac{\partial_{-} \Delta_{\mathcal{V}}}{\partial \beta}=\frac{\partial \theta_{I}^{1}}{\partial \beta}=0 \quad \text { and } \quad \frac{\partial_{+} \Delta_{\mathcal{V}}}{\partial \beta}=\frac{\partial \theta_{E}^{1}(A)}{\partial \beta}>0
$$

Next, noting that $\partial \theta_{E}^{1}(A) / \partial \pi_{I}(\ell, L)<0$ and $\partial \theta_{E}^{1}(A) / \partial \pi_{E}(L, \ell)>0$, we analogously obtain

$$
\frac{\partial_{-} \Delta_{\mathcal{V}}}{\partial \pi_{I}(\ell, L)}=\frac{\partial \theta_{E}^{1}(A)}{\partial \pi_{I}(\ell, L)}<0 \quad \text { and } \quad \frac{\partial_{+} \Delta_{\mathcal{V}}}{\partial \pi_{I}(\ell, L)}=\frac{\partial \theta_{I}^{1}}{\partial \pi_{I}(\ell, L)}=0
$$

and

$$
\frac{\partial_{-} \Delta_{\mathcal{V}}}{\partial \pi_{E}(L, \ell)}=\frac{\partial \theta_{I}^{1}}{\partial \pi_{E}(L, \ell)}=0 \quad \text { and } \quad \frac{\partial_{+} \Delta_{\mathcal{V}}}{\partial \pi_{E}(L, \ell)}=\frac{\partial \theta_{E}^{1}(A)}{\partial \pi_{E}(L, \ell)}>0
$$

\section{B.3.4 The Effect on Duplication}

Duplication is measured by the probability that both firms discover the innovation:

$$
\mathcal{D}\left(r_{I}, r_{E}\right)=\int_{0}^{1} r_{I}(\theta) r_{E}(\theta) d \theta
$$

We distinguish between equilibria with $\theta_{I}^{2}(N) \leq \theta_{I}^{1}(N)$ and $\theta_{I}^{2}(N)>\theta_{I}^{1}(N)$.

Proposition B.4 (The effect of prohibiting start-up acquisitions on duplication).

(i) When $\theta_{I}^{2}(N) \leq \theta_{I}^{1}(N)$, duplication is strictly smaller in any simple equilibrium under the no-acquisition policy than in any simple equilibrium under laissez-faire.

(ii) When $\theta_{I}^{2}(N)>\theta_{I}^{1}(N)$, there exists a threshold bargaining power $\tilde{\beta} \in[0,1)$ such that in any equilibrium under the no-acquisition policy duplication is

(a) larger than in any simple equilibrium under laissez-faire if $\beta<\tilde{\beta}$, and

(b) smaller than in any simple equilibrium under laissez-faire if $\beta>\tilde{\beta}$.

Proof. (i) First note that, under the no-acquisition policy, simple equilibria only exist when $\theta_{I}^{2}(N) \leq \theta_{I}^{1}(N)$. In any simple equilibrium under laissez-faire, $\mathcal{D}\left(r_{I}^{A}, r_{E}^{A}\right)=\theta^{2}(A)$ by Proposition 1 and in any simple equilibrium under the no-acquisition policy $\mathcal{D}\left(r_{I}^{N}, r_{E}^{N}\right)=$ 
$\theta_{E}^{2}(N)$ by Proposition B.1. For $\theta_{E}^{2}(N)<\theta^{2}(A)$, we need

$$
\begin{aligned}
C\left(\theta_{E}^{2}(N)\right) & <C\left(\theta^{2}(A)\right) \\
v_{E}^{N}(L, \ell) & <v_{E}^{A}(L, \ell) \\
\pi_{E}(L, \ell)-\kappa & <\beta\left(\max \left\{\pi_{I}(L, 0)-\kappa, \pi_{I}(\ell, 0)\right\}-\pi_{I}(\ell, L)\right)+(1-\beta)\left(\pi_{E}(L, \ell)-\kappa\right) \\
\pi_{E}(L, \ell)-\kappa & <\max \left\{\pi_{I}(L, 0)-\kappa, \pi_{I}(\ell, 0)\right\}-\pi_{I}(\ell, L)
\end{aligned}
$$

which holds by Assumption 1(iv). Hence $\mathcal{D}\left(r_{I}^{N}, r_{E}^{N}\right)<\mathcal{D}\left(r_{I}^{A}, r_{E}^{A}\right)$, which establishes part (i) of the Proposition.

(ii) We need to consider duplication in any equilibrium under the no-acquisition policy. When $\theta_{I}^{2}(N)>\theta_{I}^{1}(N)$, only non-simple equilibria exist under the no-acquisition policy. In these cases, by Proposition B.1, duplication is given by:

$$
\mathcal{D}\left(r_{I}^{N}, r_{E}^{N}\right)=\theta_{E}^{2}(N)+\int_{\max \left\{\theta_{E}^{2}(N), \theta_{I}^{1}(N)\right\}}^{\min \left\{\theta_{I}^{2}(N), \theta_{E}^{1}(N)\right\}} r_{I}^{N}(\theta) r_{E}^{N}(\theta) d \theta
$$

Now we show that there exists a threshold $\tilde{\beta}$ which determines the sign of the effect of acquisitions on duplication. When $\beta=0$, then $\theta^{2}(A)=\theta_{E}^{2}(N)$, thus

$$
\mathcal{D}\left(r_{I}^{A}, r_{E}^{A} ; \beta=0\right)-\mathcal{D}\left(r_{I}^{N}, r_{E}^{N} ; \beta=0\right)=-\int_{\max \left\{\theta_{E}^{2}(N), \theta_{I}^{1}(N)\right\}}^{\min \left\{\theta_{I}^{2}(N), \theta_{E}^{1}(N)\right\}} r_{I}^{N}(\theta) r_{E}^{N}(\theta) d \theta \leq 0 .
$$

When $\beta=1$, then $\theta^{2}(A)=\theta_{I}^{2}(N)$, thus

$$
\begin{aligned}
\mathcal{D}\left(r_{I}^{A}, r_{E}^{A} ; \beta=1\right)-\mathcal{D} & \left(r_{I}^{N}, r_{E}^{N} ; \beta=1\right)= \\
& \theta_{I}^{2}(N)-\theta_{E}^{2}(N)-\int_{\max \left\{\theta_{E}^{2}(N), \theta_{I}^{1}(N)\right\}}^{\min \left\{\theta_{I}^{2}(N), \theta_{E}^{1}(N)\right\}} r_{I}^{N}(\theta) r_{E}^{N}(\theta) d \theta>0 .
\end{aligned}
$$

The last inequality follows from the following two observations, which are implied by Proposition B.1: (i) $0 \leq \min \left\{\theta_{I}^{2}(N), \theta_{E}^{1}(N)\right\}-\max \left\{\theta_{E}^{2}(N), \theta_{I}^{1}(N)\right\} \leq \theta_{I}^{2}(N)-\theta_{E}^{2}(N)$, and (ii) $r_{I}^{N}(\theta) r_{E}^{N}(\theta) \leq 1$ for all $\theta$ and $r_{I}^{N}(\theta) r_{E}^{N}(\theta)<1$ for some $\theta$. Finally, the effect of $\beta$ on the change in duplication is monotone:

$$
\begin{aligned}
& \frac{\partial\left(\mathcal{D}\left(r_{I}^{A}, r_{E}^{A}\right)-\mathcal{D}\left(r_{I}^{N}, r_{E}^{N}\right)\right)}{\partial \beta}=\frac{\partial \theta^{2}(A)}{\partial \beta} \\
& =\frac{(1-p)\left(\max \left\{\pi_{I}(L, 0)-\kappa, \pi_{I}(\ell, 0)\right\}-\pi_{I}(\ell, L)-\pi_{E}(L, \ell)+\kappa\right)}{C^{\prime}\left(\theta^{2}(A)\right)}>0 .
\end{aligned}
$$


The intuition for the result is the following: If $\theta_{I}^{2}(N) \leq \theta_{I}^{1}(N)$, and we only consider simple equilibria in both regimes, then it is only the change in the entrant's incentive to invest in duplicate projects $\theta_{E}^{2}(N)$ which will determine the result. Similar to the effect on variety, banning acquisitions also decreases the profitability of duplicate innovations for the entrant, which leads to lower $\theta_{E}^{2}(N)$ and thus less duplication.

In the complementary case, $\theta_{I}^{2}(N)>\theta_{I}^{1}(N)$, there exists a positive measure of projects in which the incumbent invests only if this reduces the entrant's probability of receiving a patent on an $L$ innovation. This creates additional duplication for more costly projects. Allowing for acquisitions will reduce this duplication, while still increasing the duplication for rather cheap projects as discussed in the previous paragraph. The overall effect of allowing acquisitions then depends on the relative size of those countervailing effects, which is determined by the bargaining power.

The above analysis identifies two distinct reasons for duplication: Duplication because of high relative payoff of innovation, such that both investing in the same project still pays off, and duplication due to the blocking incentives of the incumbent. The latter is stronger when acquisitions are not allowed and, while duplication by the incumbent has the negative side-effect of preventing the commercialization of a new $L$ innovation and thereby competition, conversely, duplication by the entrant has the positive side-effect of increasing competition and can thus not only be considered as wasteful. However, even if duplication might be decreased by allowing acquisitions, the positive side-effect of duplication by the entrant is also shut down because his $L$ innovation will just be acquired and will not lead to more product market competition.

\section{B.4 Alternative policies}

This section contains the proofs of all four results on alternative policies (Propositions 4 to 7$)$.

\section{B.4.1 Proof of Proposition 4 (Restrictions on Technology Usage)}

Denote with $\theta_{i}^{k}(\rho)$ the critical value $k \in\{1,2\}$ of firm $i$ when the remedy is $\rho \in[0,1)$ and with $\bar{B}=\max \left\{\pi_{I}(L, 0)-\kappa, \pi_{I}(\ell, 0)\right\}-\pi_{E}(L, \ell)-\pi_{I}(\ell, L)+\kappa$ the bargaining surplus in the laissez-faire regime.

The statement in Proposition 4 on the killer-acquisitions case is obvious since the remedies do not affect the payoffs without commercialization. As to the remaining parts of Proposition 4, we prove the following statements. In the genuine-acquisitions case:

(i) If $\pi_{I}(\ell, 0) \leq \pi_{E}(L, \ell)-\kappa+\pi_{I}(\ell, L)$ and $\rho \leq \frac{\pi_{E}(L, \ell)+\pi_{I}(\ell, L)}{\pi_{I}(L, 0)}$ then $\theta_{i}^{k}(\rho)=\theta_{i}^{k}(N)$ for $k \in\{1,2\}$ and the incumbent never acquires the entrant. 
(ii) If $\pi_{I}(\ell, 0)>\pi_{E}(L, \ell)-\kappa+\pi_{I}(\ell, L)$ or $\rho>\frac{\pi_{E}(L, \ell)+\pi_{I}(\ell, L)}{\pi_{I}(L, 0)}$, then the incumbent acquires the entrant with the $L$ innovation and:

(a) $\theta_{E}^{1}(N)<\theta_{E}^{1}(\rho)<\theta_{E}^{1}(A)$;

(b) $\theta_{I}^{1}(N)=\theta_{I}^{1}(\rho)=\theta_{I}^{1}(A)$;

(c) $\theta_{E}^{2}(N)<\theta_{E}^{2}(\rho)<\theta_{E}^{2}(A)$;

(d) $\theta_{I}^{2}(N)>\theta_{I}^{2}(\rho)>\theta_{I}^{2}(A)$;

(iii) If $\pi_{I}(\ell, 0)>\pi_{E}(L, \ell)-\kappa+\pi_{I}(\ell, L)$ and $\rho \leq \frac{\pi_{I}(\ell, 0)+\kappa}{\pi_{I}(L, 0)}$, in contrast to the case without remedies, the incumbent does not commercialize the innovation after an acquisition.

Proof. Suppose that $\pi_{I}(L, 0)-\pi_{I}(\ell, 0) \geq \kappa$. We will distinguish between two cases: $\pi_{I}(\ell, 0)>\pi_{E}(L, \ell)-\kappa+\pi_{I}(\ell, L)$ and $\pi_{I}(\ell, 0) \leq \pi_{E}(L, \ell)-\kappa+\pi_{I}(\ell, L)$. A killer acquisition would increase joint surplus in the first case, but not in the second.

Case 1: $\pi_{I}(\ell, 0)>\pi_{E}(L, \ell)-\kappa+\pi_{I}(\ell, L)$.

Denote with $\bar{\rho}$ the remedies such that the incumbent is indifferent between commercializing technology $L$ and not commercializing it in case of an acquisition. That is

$$
\begin{aligned}
\bar{\rho} \pi_{I}(L, 0)-\kappa & =\pi_{I}(\ell, 0) \\
\bar{\rho} & =\frac{\pi_{I}(\ell, 0)+\kappa}{\pi_{I}(L, 0)} .
\end{aligned}
$$

Then, for any $\rho \in[0,1)$ the bargaining surplus is given by

$$
B^{\rho}= \begin{cases}\rho \pi_{I}(L, 0)-\pi_{E}(L, \ell)-\pi_{I}(\ell, L) & \text { for all } \rho \in(\bar{\rho}, 1) \\ \pi_{I}(\ell, 0)-\pi_{E}(L, \ell)-\pi_{I}(\ell, L)+\kappa & \text { for all } \rho \in[0, \bar{\rho}]\end{cases}
$$

In this case, since $\pi_{I}(\ell, 0)>\pi_{E}(L, \ell)-\kappa+\pi_{I}(\ell, L)$ and $\pi_{I}(L, 0)-\pi_{I}(\ell, 0) \geq \kappa, 0<B^{\rho}<\bar{B}$ and the acquisition takes place $\forall \rho \in(0,1)$. In contrast to Lemma 1 , the incumbent commercializes the acquired $L$ technology if and only if $\rho \in[\bar{\rho}, 1)$, while she always commercializes an $L$ technology she discovered herself. Hence, if $\rho \leq \bar{\rho}$ the incumbent acquires the entrant, but does not commercialize the innovation. This proves part $(i i i)$.

Moreover, Lemma 2 applies, with differences only in $v_{E}^{\rho}(L, \ell)=\pi_{E}(L, \ell)-\kappa+\beta B^{\rho}$ and $v_{I}^{\rho}(\ell, L)=\max \left\{\rho \pi_{I}(L, 0)-\kappa, \pi_{I}(\ell, 0)\right\}-v_{E}^{\rho}(L, \ell)$. We prove the claims $(a)-(d)$ in turn. 
(a) $\theta_{E}^{1}(N)<\theta_{E}^{1}(\rho)<\theta_{E}^{1}(A)$. This is equivalent to

$$
\begin{aligned}
C\left(\theta_{E}^{1}(N)\right) & <C\left(\theta_{E}^{1}(\rho)\right)<C\left(\theta_{E}^{1}(A)\right) \\
p v_{E}^{N}(H)+(1-p) v_{E}^{N}(L, \ell) & <p v_{E}^{\rho}(H)+(1-p) v_{E}^{\rho}(L, \ell)<p v_{E}^{A}(H)+(1-p) v_{E}^{A}(L, \ell) \\
v_{E}^{N}(L, \ell) & <v_{E}^{\rho}(L, \ell)<v_{E}^{A}(L, \ell) \\
\pi_{E}(L, \ell)-\kappa & <\pi_{E}(L, \ell)-\kappa+\beta B^{\rho}<\pi_{E}(L, \ell)-\kappa+\beta \bar{B} \\
0 & <B^{\rho}<\bar{B}
\end{aligned}
$$

which always holds.

(b) $\theta_{I}^{1}(N)=\theta_{I}^{1}(\rho)=\theta_{I}^{1}(A)$. This follows immediately by observing that $C\left(\theta_{I}^{1}\right)$ does not depend on either $\rho$ or the acquisition policy.

(c) $\theta_{E}^{2}(N)<\theta_{E}^{2}(\rho)<\theta_{E}^{2}(A)$. Observe that $C\left(\theta_{E}^{2}\right)=\frac{1}{2} C\left(\theta_{E}^{1}\right)$. The step then follows by (a) above.

(d) $\theta_{I}^{2}(N)>\theta_{I}^{2}(\rho)>\theta_{I}^{2}(A)$. This is equivalent to

$$
\begin{gathered}
C\left(\theta_{I}^{2}(N)\right)>C\left(\theta_{I}^{2}(\rho)\right)>C\left(\theta_{I}^{2}(A)\right) \\
\frac{p v_{I}^{N}(H)+(1-p)\left(v_{I}^{N}(L, 0)-v_{I}^{N}(\ell, L)\right)}{2}>\frac{p v_{I}^{\rho}(H)+(1-p)\left(v_{I}^{\rho}(L, 0)-v_{I}^{\rho}(\ell, L)\right)}{2}> \\
>\frac{p v_{I}^{A}(H)+(1-p)\left(v_{I}^{A}(L, 0)-v_{I}^{A}(\ell, L)\right)}{2} \\
-v_{I}^{N}(\ell, L)>-v_{I}^{\rho}(\ell, L)>-v_{I}^{A}(\ell, L) \\
\pi_{I}(\ell, L)<\pi_{I}(\ell, L)+(1-\beta) B^{\rho}<\pi_{I}(\ell, L)+(1-\beta) \bar{B},
\end{gathered}
$$

which always holds since $0<B^{\rho}<\bar{B}$. This proves the claim in part $(i i)$ when $\pi_{I}(\ell, 0)>$ $\pi_{E}(L, \ell)-\kappa+\pi_{I}(\ell, L)$.

Case 2: $\pi_{I}(\ell, 0) \leq \pi_{E}(L, \ell)-\kappa+\pi_{I}(\ell, L)$.

Recall that, in this case, a killer acquisition would not be worthwhile. As a genuine acquisition cannot be profitable if $\rho=0$, it will only take place if $\rho$ is sufficiently large. Denote with $\overline{\bar{\rho}}$ the remedies such that the incumbent is indifferent between acquiring and not acquiring the entrant with technology $L$, that is,

$$
\begin{aligned}
\overline{\bar{\rho}} \pi_{I}(L, 0)-\kappa & =\pi_{E}(L, \ell)-\kappa+\pi_{I}(\ell, L) \\
\overline{\bar{\rho}} & =\frac{\pi_{E}(L, \ell)+\pi_{I}(\ell, L)}{\pi_{I}(L, 0)} .
\end{aligned}
$$

In this case, the bargaining surplus is given by

$$
B^{\rho}= \begin{cases}\rho \pi_{I}(L, 0)-\pi_{E}(L, \ell)-\pi_{I}(\ell, L) & \text { for all } \rho \in(\overline{\bar{\rho}}, 1) \\ 0 & \text { for all } \rho \in[0, \overline{\bar{\rho}}]\end{cases}
$$


If $\rho>\frac{\pi_{E}(L, \ell)+\pi_{I}(\ell, L)}{\pi_{I}(L, 0)}$ as in the remaining condition of part $(i i)$, then $\rho \in(\overline{\bar{\rho}}, 1)$ and $0<$ $B^{\rho}<\bar{B}$. Then, the proof of claims $(a)-(d)$ is the same as in Case 1 above. This concludes the proof of part $(i i)$.

For part $(i)$, note when $\pi_{I}(\ell, 0) \leq \pi_{E}(L, \ell)-\kappa+\pi_{I}(\ell, L)$ and $\rho \leq \frac{\pi_{E}(L, \ell)+\pi_{I}(\ell, L)}{\pi_{I}(L, 0)}$ then $B^{\rho}=0$, by the analysis of Case 2 above. When the bargaining surplus is zero, the outcome is identical to the one when acquisitions are prohibited.

\section{B.4.2 Proof of Proposition 5 (Prohibition of "killing")}

Denote with $\theta_{i}^{k}(P K)$ the critical value $k \in\{1,2\}$ of firm $i$ when killer acquisitions are prohibited. For the statement in Proposition 5 on the genuine-acquisition case, note that in this case the incumbent acquires the entrant with $L$ technology and commercializes that technology (Lemma 1). Since "killing" never occurs, prohibiting it has no effect. To prove the remaining statements in Proposition 5, we show that (i) and (ii) below hold.

(i) If $\pi_{I}(L, 0)-\pi_{I}(\ell, 0)<\kappa$ and $\pi_{I}(L, 0)-\pi_{I}(\ell, L) \leq \pi_{E}(L, \ell)$, then $\theta_{i}^{k}(P K)=\theta_{i}^{k}(N)$ for $k \in\{1,2\}$ and the incumbent never acquires the entrant.

(ii) If $\pi_{I}(L, 0)-\pi_{I}(\ell, 0)<\kappa$ and $\pi_{I}(L, 0)-\pi_{I}(\ell, L)>\pi_{E}(L, \ell)$, then:

(a) $\theta_{E}^{1}(N)<\theta_{E}^{1}(P K)<\theta_{E}^{1}(A)$;

(b) $\theta_{I}^{1}(N)=\theta_{I}^{1}(P K)=\theta_{I}^{1}(A)$;

(c) $\theta_{E}^{2}(N)<\theta_{E}^{2}(P K)<\theta_{E}^{2}(A)$;

(d) $\theta_{I}^{2}(N)>\theta_{I}^{2}(P K)>\theta_{I}^{2}(A)$;

The incumbent acquires the entrant with the $\mathrm{L}$ innovation and commercializes it after the acquisition.

Proof. (i) If the incumbent acquires the entrant, she has to commercialize the technology. The total surplus is $\pi_{I}(L, 0)-\kappa$. Without the acquisition, the total surplus is equal to $\pi_{I}(\ell, L)+\pi_{E}(L, \ell)-\kappa$. Since $\pi_{I}(L, 0)-\pi_{I}(\ell, L) \leq \pi_{E}(L, \ell)$, the bargaining surplus is weakly negative, so that an acquisition never materializes and the outcome is identical to the one when acquisitions are prohibited.

(ii) Since $\pi_{I}(L, 0)-\pi_{I}(\ell, L)>\pi_{E}(L, \ell)$ the bargaining surplus $B^{P K}=\pi_{I}(L, 0)-$ $\pi_{I}(\ell, L)-\pi_{E}(L, \ell)>0$, so that the incumbent acquires the entrant with the $L$ technology. Denote the bargaining surplus in the laissez-faire regime $\bar{B}=\max \left\{\pi_{I}(L, 0)-\kappa, \pi_{I}(\ell, 0)\right\}-$ $\pi_{E}(L, \ell)-\pi_{I}(\ell, L)+\kappa$ and note that $\bar{B}>B^{P K}$. The proofs of claims (a)-(d) are completely analogous to those of claims (a)-(d) in the subsection above and are omitted. 


\section{B.4.3 Proof of Proposition 6 (Taxing acquisitions)}

Denote with $\theta_{i}^{k}(\tau)$ the critical value $k \in\{1,2\}$ of firm $i$ when the tax rate is $\tau$. As before, let $\bar{B}=\max \left\{\pi_{I}(L, 0)-\kappa, \pi_{I}(\ell, 0)\right\}-\pi_{E}(L, \ell)-\pi_{I}(\ell, L)+\kappa$, denote the bargaining surplus in the laissez-faire regime. Denote the bargaining surplus when the tax rate is $\tau$ with $B^{\tau}$. The acquisition price is $\pi_{E}(L, \ell)-\kappa+\beta B^{\tau}$. Since the initial bargaining surplus is reduced by the total amount of tax paid, the following equality has to be satisfied

$$
B^{\tau}=\bar{B}-\tau\left(\pi_{E}(L, \ell)-\kappa+\beta B^{\tau}\right)
$$

so that

$$
B^{\tau}=\frac{\bar{B}-\tau\left(\pi_{E}(L, \ell)-\kappa\right)}{1+\tau \beta} .
$$

The following statements imply Proposition 6:

(i) If $\tau \geq \frac{\bar{B}}{\pi_{E}(L, \ell)-\kappa}$, then $\theta_{i}^{k}(\tau)=\theta_{i}^{k}(N)$ for $k \in\{1,2\}$ and the incumbent never acquires the entrant

(ii) If $0<\tau<\frac{\bar{B}}{\pi_{E}(L, \ell)-\kappa}$ then the incumbent acquires the entrant with the $\mathrm{L}$ innovation and:

(a) $\theta_{E}^{1}(N)<\theta_{E}^{1}(\tau)<\theta_{E}^{1}(A)$

(b) $\theta_{I}^{1}(N)=\theta_{I}^{1}(\tau)=\theta_{I}^{1}(A)$;

(c) $\theta_{E}^{2}(N)<\theta_{E}^{2}(\tau)<\theta_{E}^{2}(A)$;

(d) $\theta_{I}^{2}(N)>\theta_{I}^{2}(\tau)>\theta_{I}^{2}(A)$.

Proof. Consider part (i), so that $\tau \geq \frac{\bar{B}}{\pi_{E}(L, \ell)-\kappa}$. Then, $B^{\tau}=\frac{\bar{B}-\tau\left(\pi_{E}(L, \ell)-\kappa\right)}{1+\tau \beta} \leq 0$. Hence, no acquisitions will take place, so that the outcome is the same as in the game where acquisitions are prohibited.

Now suppose that $0<\tau<\frac{\bar{B}}{\pi_{E}(L, \ell)-\kappa}$. Then, $B^{\tau}=\frac{\bar{B}-\tau\left(\pi_{E}(L, \ell)-\kappa\right)}{1+\tau \beta}>0$. Therefore, the bargaining surplus is positive and Lemma 1 holds. Lemma 2 holds as well, except for the values when the acquisition takes place. Now, $v_{E}^{\tau}(L, \ell)=\pi_{E}(L, \ell)-\kappa+\beta B^{\tau}$ and $v_{I}^{\tau}(\ell, L)=v_{I}(L, 0)-(1+\tau) v_{E}^{\tau}(L, \ell)$.

We prove the claims in part (ii) in turn.

(a) $\theta_{E}^{1}(N)<\theta_{E}^{1}(\tau)<\theta_{E}^{1}(A)$. 
This is equivalent to

$$
\begin{aligned}
C\left(\theta_{E}^{1}(N)\right) & <C\left(\theta_{E}^{1}(\tau)\right)<C\left(\theta_{E}^{1}(A)\right) \\
p v_{E}^{N}(H)+(1-p) v_{E}^{N}(L, \ell) & <p v_{E}^{\tau}(H)+(1-p) v_{E}^{\tau}(L, \ell)<p v_{E}^{A}(H)+(1-p) v_{E}^{A}(L, \ell) \\
v_{E}^{N}(L, \ell) & <v_{E}^{\tau}(L, \ell)<v_{E}^{A}(L, \ell) \\
\pi_{E}(L, \ell)-\kappa & <\pi_{E}(L, \ell)-\kappa+\beta B^{\tau}<\pi_{E}(L, \ell)-\kappa+\beta \bar{B} \\
0 & <B^{\tau}<\bar{B}
\end{aligned}
$$

which always holds for the values $\tau$ takes in the case examined.

(b) $\theta_{I}^{1}(N)=\theta_{I}^{1}(\tau)=\theta_{I}^{1}(A)$.

This follows immediately by observing that $C\left(\theta_{I}^{1}\right)$ does not depend on either $\tau$ or the acquisition policy.

(c) $\theta_{E}^{2}(N)<\theta_{E}^{2}(\tau)<\theta_{E}^{2}(A)$.

Observe that $C\left(\theta_{E}^{2}\right)=\frac{1}{2} C\left(\theta_{E}^{1}\right)$. The step then follows by Step 1 above.

(d) $\theta_{I}^{2}(N)>\theta_{I}^{2}(\tau)>\theta_{I}^{2}(A)$.

This is equivalent to

$$
\begin{gathered}
C\left(\theta_{I}^{2}(N)\right)>C\left(\theta_{I}^{2}(\tau)\right)>C\left(\theta_{I}^{2}(A)\right) \\
\frac{p v_{I}^{N}(H)+(1-p)\left(v_{I}^{N}(L, 0)-v_{I}^{N}(\ell, L)\right)}{2}>\frac{p v_{I}^{\tau}(H)+(1-p)\left(v_{I}^{\tau}(L, 0)-v_{I}^{\tau}(\ell, L)\right)}{2}> \\
>\frac{p v_{I}^{A}(H)+(1-p)\left(v_{I}^{A}(L, 0)-v_{I}^{A}(\ell, L)\right)}{2} \\
-v_{I}^{N}(\ell, L)>-v_{I}^{\tau}(\ell, L)>-v_{I}^{A}(\ell, L) \\
\pi_{I}(\ell, L)<\pi_{I}(\ell, L)+(1-\beta) B^{\tau}<\pi_{I}(\ell, L)+(1-\beta) \bar{B},
\end{gathered}
$$

which always holds since $0<B^{\tau}<\bar{B}$.

\section{B.4.4 Proof of Proposition 7 (Increasing Profitability of IPOs)}

Consider a policy with preferential treatment of IPOs. We operationalize this policy by supposing that the net profit of the entrant is given by $\eta \pi_{E}(H)$ and $\eta \pi_{E}(L, \ell)$, where $\eta=1$ represents the status quo and $\eta>1$ represents the policy of preferential IPO treatment. Denote with $\theta_{i}^{k}(\eta)$ the critical value $k \in\{1,2\}$ of firm $i$ when the IPO policy is $\eta>1$. As before, let $\bar{B}=\max \left\{\pi_{I}(L, 0)-\kappa, \pi_{I}(\ell, 0)\right\}-\pi_{E}(L, \ell)-\pi_{I}(\ell, L)+\kappa$, denote the bargaining surplus in the laissez-faire regime. The following statements imply Proposition 7:

(i) For any $\eta>1$ :
(a) $\theta_{E}^{1}(A)<\theta_{E}^{1}(\eta)$;
(b) $\theta_{I}^{1}(A)=\theta_{I}^{1}(\eta)$;
(c) $\theta_{E}^{2}(A)<\theta_{E}^{2}(\eta)$;
(d) $\theta_{I}^{2}(A)<\theta_{I}^{2}(\eta)$. 
(ii) The incumbent acquires the entrant if and only if

$$
\eta<\frac{\bar{B}+\pi_{E}(L, \ell)}{\pi_{E}(L, \ell)}
$$

Proof. First, note that the measure does not affect the commercialization decision of the entrant or the incumbent. Next, the incumbent acquires the entrant if and only if the entrant has technology $L$ and

$$
\begin{aligned}
\eta \pi_{E}(L, \ell)+\pi_{I}(\ell, L)-\kappa & <\max \left\{\pi_{I}(L, 0)-\kappa, \pi_{I}(\ell, 0)\right\} \\
\eta & <\frac{\max \left\{\pi_{I}(L, 0)-\kappa, \pi_{I}(\ell, 0)\right\}-\pi_{I}(\ell, L)+\kappa}{\pi_{E}(L, \ell)} \\
\eta & <\frac{\bar{B}+\pi_{E}(L, \ell)}{\pi_{E}(L, \ell)} .
\end{aligned}
$$

The entrant's values after the realization of the innovation outcomes are

$$
\begin{aligned}
v_{E}^{\eta}(H)= & \eta \pi(H)-\kappa \\
v_{E}^{\eta}(L, \ell)= & \eta \pi_{E}(L, \ell)-\kappa+ \\
& +\beta \max \left\{0,\left(\max \left\{\pi_{I}(L, 0)-\kappa, \pi_{I}(\ell, 0)\right\}-\eta \pi_{E}^{\eta}(L, \ell)-\pi_{I}(\ell, L)+\kappa\right)\right\} \\
v_{E}^{\eta}\left(0, t_{I}\right)= & 0 \text { for } t_{I} \in\{\ell, L, H\} .
\end{aligned}
$$

The incumbent's values after the realization of the innovation outcomes are

$$
\begin{aligned}
& v_{I}^{\eta}(H)=\pi(H)-\kappa \\
& v_{I}^{\eta}(L, 0)=\max \left\{\pi_{I}(L, 0)-\kappa, \pi_{I}(\ell, 0)\right\} \\
& v_{I}^{\eta}(\ell, L)=\pi_{I}(\ell, L)+ \\
& +(1-\beta) \max \left\{0,\left(\max \left\{\pi_{I}(L, 0)-\kappa, \pi_{I}(\ell, 0)\right\}-\eta \pi_{E}^{\eta}(L, \ell)-\pi_{I}(\ell, L)+\kappa\right)\right\} \\
& v_{I}^{\eta}(\ell, 0)=\pi_{I}(\ell, 0) \\
& v_{I}^{\eta}(\ell, H)=0 \text {. }
\end{aligned}
$$

We prove the claims $(a)-(d)$ in the proposition in turn.

(a) $\theta_{E}^{1}(A)<\theta_{E}^{1}(\eta)$.

This is equivalent to

$$
\begin{gathered}
C\left(\theta_{E}^{1}(A)\right)<C\left(\theta_{E}^{1}(\eta)\right) \\
p v_{E}^{A}(H)+(1-p) v_{E}^{A}(L, \ell)<p v_{E}^{\eta}(H)+(1-p) v_{E}^{\eta}(L, \ell)
\end{gathered}
$$

which holds since $v_{E}^{A}(H)<v_{E}^{\eta}(H)$ and $v_{E}^{A}(L, \ell)<v_{E}^{\eta}(L, \ell)$.

(b) $\theta_{I}^{1}(A)=\theta_{I}^{1}(\eta)$.

This follows immediately by observing that $C\left(\theta_{I}^{1}\right)$ is not affected by $\eta$.

(c) $\theta_{E}^{2}(A)<\theta_{E}^{2}(\eta)$.

Observe that $C\left(\theta_{E}^{2}\right)=\frac{1}{2} C\left(\theta_{E}^{1}\right)$. The step then follows by (a) above.

(d) $\theta_{I}^{2}(A)<\theta_{I}^{2}(\eta)$. 
This is equivalent to

$$
\begin{aligned}
C\left(\theta_{I}^{2}(A)\right) & <C\left(\theta_{I}^{2}(\eta)\right) \\
\frac{p v_{I}^{A}(H)+(1-p)\left(v_{I}^{A}(L, 0)-v_{I}^{A}(\ell, L)\right)}{2} & <\frac{p v_{I}^{\eta}(H)+(1-p)\left(v_{I}^{\eta}(L, 0)-v_{I}^{\eta}(\ell, L)\right)}{2} \\
v_{I}^{A}(\ell, L) & >v_{I}^{\eta}(\ell, L),
\end{aligned}
$$

which always holds since the incumbent's bargaining surplus is lower when $\eta>1$. That is

$$
\begin{aligned}
\max \left\{\pi_{I}(L, 0)-\kappa, \pi_{I}(\ell, 0)\right\}-\pi_{E}(L, \ell)-\pi_{I}(\ell, L)+\kappa \\
>\max \left\{0,\left(\max \left\{\pi_{I}(L, 0)-\kappa, \pi_{I}(\ell, 0)\right\}-\eta \pi_{E}^{\eta}(L, \ell)-\pi_{I}(\ell, L)+\kappa\right)\right\} .
\end{aligned}
$$

\section{B.5 Robustness Results}

This section contains precise statements of the robustness claims of Section 7 as well as proofs of these results.

\section{B.5.1 Innovation Uncertainty at the Time of Acquisition}

The new timeline leads to the following result in the acquisition subgame:

Lemma B.2 (Acquisitions). Suppose at the time of the acquisition the technology level of the innovation is uncertain. The incumbent acquires the entrant if and only if the entrant holds a patent for the innovation. After the acquisition, the incumbent always commercializes the $H$ technology. She commercializes the L technology if and only if $\pi(L, 0)-\pi(\ell, 0) \geq \kappa$.

Proof. First, suppose that the entrant holds no patent. Then, since the entrant cannot compete without an innovation, the incumbent's profits are the same with or without the acquisition. Thus, the incumbent has no reason to acquire the entrant.

Second, suppose the entrant holds a patent. Without an acquisition, the entrant commercializes the technology irrespective of the realized technology level according to Assumption 2. He thus obtains the expected payoff $p(\pi(H)-\kappa)+(1-p)\left(\pi_{E}(L, \ell)-\kappa\right)$ while the incumbent obtains $(1-p) \pi_{I}(\ell, L)$. With the acquisition, the incumbent commercializes the $H$ technology according to Assumption 2, but only commercializes the $L$ technology if $\pi_{I}(L, 0)-\kappa \geq \pi_{I}(\ell, 0)$. Thus, the incumbent's expected payoff is $p(\pi(H)-\kappa)+(1-$ $p)\left(\max \left\{\pi_{I}(L, 0)-\kappa, \pi_{I}(\ell, 0)\right\}\right)$. The entrant obtains a payoff of zero. Consequently, the 
expected acquisition surplus is

$$
(1-p)\left[\max \left\{\pi_{I}(L, 0)-\kappa, \pi_{I}(\ell, 0)\right\}-\pi_{E}(L, \ell)-\pi_{I}(\ell, L)+\kappa\right]
$$

The acquisition surplus is positive if and only if $\max \left\{\pi_{I}(L, 0)-\kappa, \pi_{I}(\ell, 0)\right\}>\pi_{E}(L, \ell)+$ $\pi_{I}(\ell, L)-\kappa$, which holds by Assumption 1(iv).

Under the conditions of B.2 acquisitions happen more frequently than in the case of 1. Not only does the incumbent acquire the entrant if his innovation turns out to be non-drastic, but also if the entrant's innovation turns out to be drastic. Thus, the entrant will never enter the market, neither as competitor nor as new monopolist. However, he will be compensated for the possibility that his innovation may turn out to be drastic.

Proposition B.5. With uncertainty at the time of acquisition, any investment equilibrium under the alternative timeline with uncertainty is an investment equilibrium under the original timeline without uncertainty and vice versa.

Proof. As in the main model, the equilibrium investment behavior will depend on the critical projects, for which the respective firm $E$ or $I$ is just indifferent between investing and not investing conditional on the behavior of the rival. Since, to be indifferent, payoffs need to equal investment costs, we will first introduce the new values $\tilde{v}_{i}$ for each firm $i \in\{I, E\}$ at the beginning of the acquisition stage in the laissez-faire regime, depending on whether the firm owns a patent, $t_{i}^{\text {int }} \in\{0,1\}$ :

Lemma B.3 (Payoffs).

In the case with uncertainty at the time of acquisition, consider the laissez-faire policy.

(i) The entrant's values after the realization of the innovation results are

$$
\begin{aligned}
& \tilde{v}_{E}(1,0)=p \pi(H)+(1-p) \pi_{E}(L, \ell)-\kappa+\beta(1-p)\left(\max \left\{\pi_{I}(L, 0)-\kappa, \pi_{I}(\ell, 0)\right\}-\right. \\
& \left.\pi_{E}(L, \ell)-\pi_{I}(\ell, L)+\kappa\right) \\
& \tilde{v}_{E}\left(0, t_{I}^{\text {int }}\right)=0 \text { for } t_{I}^{\text {int }} \in\{0,1\} .
\end{aligned}
$$

(ii) The incumbent's values after the realization of the innovation results are

$$
\begin{aligned}
& \tilde{v}_{I}(1,0)=p(\pi(H)-\kappa)+(1-p) \max \left\{\pi_{I}(L, 0)-\kappa, \pi_{I}(\ell, 0)\right\} \\
& \tilde{v}_{I}(0,1)=p(\pi(H)-\kappa)+(1-p) \max \left\{\pi_{I}(L, 0)-\kappa, \pi_{I}(\ell, 0)\right\}-\tilde{v}_{E}(1,0) \\
& \tilde{v}_{I}(0,0)=\pi_{I}(\ell, 0)
\end{aligned}
$$

We will refer to the critical thresholds under the alternative timeline, and thus new values, as $\tilde{\theta}_{i}^{1}, \tilde{\theta}_{i}^{2}, i \in\{E, I\}$. It turns out that these critical projects are identical to their 
counterparts in the original timeline without uncertainty:

$$
\begin{aligned}
C\left(\tilde{\theta}_{E}^{1}\right)= & \tilde{v}_{E}(1,0) \\
= & p \pi(H)+(1-p) \pi_{E}(L, \ell)-\kappa+ \\
& \beta(1-p)\left(\max \left\{\pi_{I}(L, 0)-\kappa, \pi_{I}(\ell, 0)\right\}-\pi_{E}(L, \ell)-\pi_{I}(\ell, L)+\kappa\right) \\
= & p(\pi(H)-\kappa)+(1-p)\left(\pi_{E}(L, \ell)-\kappa+\right. \\
& \left.\beta\left(\max \left\{\pi_{I}(L, 0)-\kappa, \pi_{I}(\ell, 0)\right\}-\pi_{E}(L, \ell)-\pi_{I}(\ell, L)+\kappa\right)\right) \\
= & p v_{E}(H)+(1-p) v_{E}(L, \ell)=C\left(\theta_{E}^{1}\right) \\
C\left(\tilde{\theta}_{E}^{2}\right)= & \frac{1}{2} \tilde{v}_{E}(1,0)=\frac{1}{2} p v_{E}(H)+(1-p) v_{E}(L, \ell)=\frac{1}{2} C\left(\theta_{E}^{1}\right)=C\left(\theta_{E}^{2}\right) \\
C\left(\tilde{\theta}_{I}^{1}\right)= & \tilde{v}_{I}(1,0)-\tilde{v}_{I}(0,0) \\
= & p(\pi(H)-\kappa)+(1-p) \max \left\{\pi_{I}(L, 0)-\kappa, \pi_{I}(\ell, 0)\right\}-\pi_{I}(\ell, 0) \\
= & p v_{I}(H)+(1-p) v_{I}(L, 0)-v_{I}(\ell, 0)=C\left(\theta_{I}^{1}\right) \\
C\left(\tilde{\theta}_{I}^{2}\right)= & \frac{1}{2} \tilde{v}_{I}(1,0)+\frac{1}{2} \tilde{v}_{I}(0,1)-\tilde{v}_{I}(0,1)=\frac{1}{2} v_{E}(1,0) \\
= & \frac{1}{2}\left(p \pi(H)+(1-p) \pi_{E}(L, \ell)-\kappa+\right. \\
& \left.\beta(1-p)\left(\max \left\{\pi_{I}(L, 0)-\kappa, \pi_{I}(\ell, 0)\right\}-\pi_{E}(L, \ell)-\pi_{I}(\ell, L)+\kappa\right)\right) \\
= & \frac{1}{2}\left(p v_{I}(H)+(1-p) v_{E}(L, \ell)\right)=C\left(\theta_{I}^{2}\right) .
\end{aligned}
$$

Since projects costs are strictly increasing in $\theta$, equality of costs establishes equality of the values themselves, i.e. $\tilde{\theta}_{i}^{1}=\theta_{i}^{1}$ and $\tilde{\theta}_{i}^{2}=\theta_{i}^{2}$ for $i \in\{I, E\}$.

Again, under the no-acquisition policy, only two values change, $v_{E}(1,0)=p \pi(H)+$ $(1-p) \pi_{E}(L, \ell)-\kappa$ and $v_{I}(0,1)=(1-p) \pi_{I}(\ell, L)$. Moreover, it is easy to see that the critical values are identical irrespective of which timeline we assume. Recall that according to Proposition A.1, the relative position of critical values is sufficient for the construction of equilibrium research strategies.

Proposition B.5 implies that equilibrium research strategies in the two policy regimes do not depend on whether there is uncertainty at the time of acquisition. Moreover, we can apply Propositions 2 to evaluate the effect of prohibiting acquisitions. Since the effect is solely based on the research strategies, it is not affected by the amount of the uncertainty at the time of acquisition. 


\section{B.5.2 Asymmetric Chances of Receiving Patents}

We now prove the following result.

Proposition B.6. Consider the case with asymmetric patenting probabilities $\alpha_{I} \in(0,1)$ and $\alpha_{E}=1-\alpha_{I}$.

(i) In any equilibrium $\left(r_{I}^{N}, r_{E}^{N}\right)$ under the no-acquisition policy, (a) the variety of research projects is weakly smaller than in any equilibrium $\left(r_{I}^{A}, r_{E}^{A}\right)$ under the laissez-faire policy; and (b) the probability of an innovation is weakly smaller than in any simple equilibrium $\left(r_{I}^{A}, r_{E}^{A}\right)$ under the laissez-faire policy.

(ii) The policy effect $\Delta_{\mathcal{V}}=\mathcal{V}^{A}-\mathcal{V}^{N}$ is independent of $\alpha_{I} \in(0,1)$.

The subgames after the end of the investment stage are the same as in the main model, so that the continuation values under the laissez-faire policy are given by Lemma 2 , and under the no-acquisition policy they are the same as in Lemma 2 except that $v_{I}^{N}(\ell, L)=\pi_{I}(\ell, L)$ and $v_{E}^{N}(L, \ell)=\pi_{E}(L, \ell)-\kappa$ (as in the main model). In addition, the critical projects $\theta_{I}^{1}$ and $\theta_{E}^{1}$ do not depend on $\alpha$; so that their definition in Section 3 still applies. However, $\alpha_{I}$ affects the critical projects $\theta_{I}^{2}$ and $\theta_{E}^{2}$ and thus the equilibrium investments $r_{i}$. Denote the critical project $\theta_{I}^{2}$ under the policy $A$ for the given $\alpha_{I}$ as $\theta_{E}^{2}\left(A, \alpha_{I}\right)$, and similarly for the other critical projects. Under laissez-faire,

$$
\begin{aligned}
C\left(\theta_{E}^{2}\left(A, \alpha_{I}\right)\right) & =\left(1-\alpha_{I}\right)\left(p v_{E}(H)+(1-p) v_{E}(L, \ell)\right) \\
C\left(\theta_{I}^{2}\left(A, \alpha_{I}\right)\right) & =p \alpha_{I} v_{I}(H)+(1-p)\left(\alpha_{I} v_{I}(L, 0)+\left(1-\alpha_{I}\right) v_{I}(\ell, L)\right)-(1-p) v_{I}(\ell, L) .
\end{aligned}
$$

First, note that $\theta_{E}^{1}(A)>\theta_{E}^{2}\left(A, \alpha_{I}\right)$ for all $\alpha_{I} \in(0,1)$. Furthermore, since $\theta_{E}^{1}(A)$ and $\theta_{I}^{1}(A)$ do not depend on $\alpha_{I}$, the following result follows directly (by arguments which are standard by now).

Lemma B.4. Fix any $\alpha_{I} \in(0,1)$. Under the laissez-faire policy, in any equilibrium, $\mathcal{V}^{A}=\max \left\{\theta_{E}^{1}(A), \theta_{I}^{1}(A)\right\}$.

The critical projects under the no-acquisition policy, $\theta_{I}^{1}(N)$ and $\theta_{E}^{1}(N)$, are given as in Section 3 and thus are independent of $\alpha_{I} . \theta_{I}^{2}\left(N, \alpha_{I}\right)$ and $\theta_{E}^{2}\left(N, \alpha_{I}\right)$ are defined implicitly as follows:

$$
\begin{aligned}
C\left(\theta_{E}^{2}\left(N, \alpha_{I}\right)\right) & =\left(1-\alpha_{I}\right)\left(p v_{E}(H)+(1-p) v_{E}^{N}(L, \ell)\right) \\
C\left(\theta_{I}^{2}\left(N, \alpha_{I}\right)\right) & =p \alpha_{I} v_{I}(H)+(1-p)\left(\alpha_{I} v_{I}(L, 0)+\left(1-\alpha_{I}\right) v_{I}^{N}(\ell, L)\right)-(1-p) v_{I}^{N}(\ell, L) .
\end{aligned}
$$

Again, note that $\theta_{E}^{1}(N)>\theta_{E}^{2}\left(N, \alpha_{I}\right)$ for all $\alpha_{I} \in(0,1)$ and thus, since $\theta_{E}^{1}(N)$ and $\theta_{I}^{1}(N)$ do not depend on $\alpha_{I}$, it follows directly that: 
Lemma B.5. Fix any $\alpha_{I} \in(0,1)$. Under the laissez-faire policy, in any equilibrium, $\mathcal{V}^{N}=\max \left\{\theta_{E}^{1}(N), \theta_{I}^{1}(N)\right\}$.

Therefore, neither $\mathcal{V}^{A}$ nor $\mathcal{V}^{N}$ depend on $\alpha_{I}$, proving Proposition B.6.

\section{B.5.3 Heterogeneous Commercialization Costs}

We now prove the following result.

Proposition B.7. Suppose $\kappa_{I}<\kappa_{E} \leq \pi_{E}(L, \ell)$ and $\pi_{I}(L, 0)-\pi_{I}(\ell, 0)<\kappa_{I} \leq \pi(H)-$ $\pi_{I}(\ell, 0)$. In any equilibrium $\left(r_{I}^{N}, r_{E}^{N}\right)$ under the no-acquisition policy,

(i) the variety of research projects is strictly smaller than in any equilibrium $\left(r_{I}^{A}, r_{E}^{A}\right)$ under the laissez-faire policy;

(ii) the probability of an innovation is strictly smaller than in any simple equilibrium $\left(r_{I}^{A}, r_{E}^{A}\right)$ under the laissez-faire policy.

Solving the game backwards, we first characterize the behavior of the firms in the commercialization and acquisition subgames.

Lemma B.6. In the model with heterogeneous commercialization costs, the incumbent acquires the entrant whenever the entrant holds a patent for any technology. The incumbent commercializes only the technology $H$. The entrant commercializes both technologies.

Proof. Since by assumption $\pi_{I}(L, 0)-\pi_{I}(\ell, 0)<\kappa_{I} \leq \pi(H)-\pi_{I}(\ell, 0)$, the incumbent commercializes only the $H$ technology. Since $\pi(H)>\pi_{E}(L, \ell) \geq \kappa_{E}$, the entrant commercializes both technologies. In the acquisition stage, if the entrant does not hold a patent, there is no reason for the acquisition. If the entrant holds a patent for the $H$ technology, joint profits strictly increase after the acquisition, since $\pi(H)-\kappa_{I}>\pi(H)-\kappa_{E}$. Hence, the incumbent acquires the entrant. If the entrant holds a patent for the $L$ technology, joint profits strictly increase after the acquisition, since $\pi_{I}(\ell, 0)>\pi_{E}(L, \ell)-\kappa_{E}+\pi_{I}(\ell, L)$, which holds by Assumption 1. Hence, the incumbent acquires the entrant.

Under the laissez-faire policy, the continuation payoffs are given below.

(i) The entrant's values after the realization of the innovation results are

$$
\begin{aligned}
& v_{E}(H)=\pi(H)-\kappa_{E}+\beta\left(\kappa_{E}-\kappa_{I}\right) \\
& v_{E}(L, \ell)=\pi_{E}(L, \ell)-\kappa_{E}+\beta\left(\pi_{I}(\ell, 0)-\pi_{E}(L, \ell)-\pi_{I}(\ell, L)+\kappa_{E}\right) \\
& v_{E}\left(0, t_{I}\right)=0 \text { for } t_{I} \in\{\ell, L, H\} .
\end{aligned}
$$

(ii) The incumbent's values after the realization of the innovation results are

$$
\begin{aligned}
& v_{I}(H)=\pi(H)-\kappa_{I} \\
& v_{I}(L, 0)=v_{I}(\ell, 0)=\pi_{I}(\ell, 0) \\
& v_{I}(\ell, L)=\pi_{I}(\ell, 0)-v_{E}(L, \ell) \\
& v_{I}(\ell, H)=\pi(H)-\kappa_{I}-v_{E}(H)=(1-\beta)\left(\kappa_{E}-\kappa_{I}\right) .
\end{aligned}
$$


Using these continuation values to calculate the critical values, we immediately obtain that $\theta_{E}^{2}(A)<\theta_{E}^{1}(A)$. Next, $\theta_{I}^{1}(A)<\theta_{E}^{1}(A)$ if and only if

$$
\begin{aligned}
C\left(\theta_{I}^{1}(A)\right) & <C\left(\theta_{E}^{1}(A)\right) \\
p v_{I}(H)+(1-p) v_{I}(L, 0)-v_{I}(\ell, 0) & <p v_{E}(H)+(1-p) v_{E}(L, \ell) \\
p\left(\pi(H)-\kappa_{I}\right)-p \pi(\ell, 0) & <p\left(\pi(H)-\kappa_{E}+\beta\left(\kappa_{E}-\kappa_{I}\right)\right)+(1-p) v_{E}(L, \ell) \\
p\left((1-\beta)\left(\kappa_{E}-\kappa_{I}\right)-\pi(\ell, 0)\right) & <(1-p) v_{E}(L, \ell) .
\end{aligned}
$$

Since $v_{E}(L, \ell)>0$, for the above to hold it is sufficient that $\kappa_{E}-\kappa_{I} \leq \pi_{I}(\ell, 0)$. Since $\pi_{E}(L, \ell) \geq \kappa_{E}$ and $\pi_{I}(L, 0)-\pi_{I}(\ell, 0)<\kappa_{I}$ by assumption, then $\kappa_{E}-\kappa_{I}<\pi_{E}(L, \ell)-$ $\left(\pi_{I}(L, 0)-\pi_{I}(\ell, 0)\right)$. Furthermore, $\pi_{E}(L, \ell) \leq \pi_{I}(L, 0)$ implies that $\pi_{E}(L, \ell)-\left(\pi_{I}(L, 0)-\right.$ $\left.\pi_{I}(\ell, 0)\right) \leq \pi_{I}(\ell, 0)$, so that $\kappa_{E}-\kappa_{I}<\pi_{I}(\ell, 0)$ always holds. Therefore, $\theta_{I}^{1}(A)<\theta_{E}^{1}(A)$ is always satisfied.

Together, this implies that $\theta_{E}^{1}(A)>\max \left\{\theta_{E}^{2}(A), \theta_{I}^{1}(A)\right\}$, which leads (by arguments which are standard by now) to the following result.

Lemma B.7. Suppose that $\kappa_{I}<\kappa_{E} \leq \pi_{E}(L, \ell)$ and $\pi_{I}(L, 0)-\pi_{I}(\ell, 0)<\kappa_{I} \leq \pi(H)-$ $\pi_{I}(\ell, 0)$. Then, in any equilibrium under the laissez-faire policy, $\mathcal{V}^{A}=\theta_{E}^{1}(A)$.

Next, we analyze the no-acquisition policy. The continuation payoffs are given below.

(i) The entrant's values after the realization of the innovation results are

$$
\begin{aligned}
& v_{E}(H)=\pi(H)-\kappa_{E} \\
& v_{E}(L, \ell)=\pi_{E}(L, \ell)-\kappa_{E} \\
& v_{E}\left(0, t_{I}\right)=0 \text { for } t_{I} \in\{\ell, L, H\} .
\end{aligned}
$$

(ii) The incumbent's values after the realization of the innovation results are

$$
\begin{aligned}
& v_{I}(H)=\pi(H)-\kappa_{I} \\
& v_{I}(L, 0)=v_{I}(\ell, 0)=\pi_{I}(\ell, 0) \\
& v_{I}(\ell, L)=\pi_{I}(\ell, L) \\
& v_{I}(\ell, H)=0 .
\end{aligned}
$$

As before, it is immediate that $\theta_{E}^{2}(N)<\theta_{E}^{1}(N)$. Next, $\theta_{I}^{1}(N) \leq \theta_{E}^{1}(N)$ if and only if

$$
\begin{aligned}
C\left(\theta_{I}^{1}(N)\right) & \leq C\left(\theta_{E}^{1}(N)\right) \\
p v_{I}(H)+(1-p) v_{I}(L, 0)-v_{I}(\ell, 0) & \leq p v_{E}(H)+(1-p) v_{E}(L, \ell) \\
p\left(\pi(H)-\kappa_{I}\right)-p \pi_{I}(\ell, 0) & \leq p\left(\pi(H)-\kappa_{E}\right)+(1-p)\left(\pi_{E}(L, \ell)-\kappa_{E}\right) \\
-p \kappa_{I}-p \pi_{I}(\ell, 0) & \leq(1-p) \pi_{E}(L, \ell)-\kappa_{E} .
\end{aligned}
$$

For this inequality to hold, it is sufficient that

$$
-p\left(\pi_{I}(L, 0)-\pi_{I}(\ell, 0)\right)-p \pi_{I}(\ell, 0) \leq(1-p) \pi_{E}(L, \ell)-\pi_{E}(L, \ell)
$$




$$
\begin{aligned}
-p \pi_{I}(L, 0) & \leq-p \pi_{E}(L, \ell) \\
\pi_{I}(L, 0) & \geq \pi_{E}(L, \ell)
\end{aligned}
$$

which is satisfied by assumption. Therefore, $\theta_{E}^{1}(N) \geq \max \left\{\theta_{E}^{2}(N), \theta_{I}^{1}(N)\right\}$, which leads (by arguments which are standard by now) to the following result.

Lemma B.8. Suppose $\kappa_{I}<\kappa_{E} \leq \pi_{E}(L, \ell)$ and $\pi_{I}(L, 0)-\pi_{I}(\ell, 0)<\kappa_{I} \leq \pi(H)-\pi_{I}(\ell, 0)$. Then, in any equilibrium under the no-acquisition policy, $\mathcal{V}^{N}=\theta_{E}^{1}(N)$.

Since $\theta_{E}^{1}(N)<\theta_{E}^{1}(A)$, the two lemmas in this section prove Proposition B.7.

\section{B.5.4 Heterogeneous Innovation Outcomes}

Denote the expected net payoff of investing in project $\theta$ conditional on the other firm not investing as $R_{i}^{1}(\theta)$ and the expected net payoff of investing in project $\theta$ conditional on the other firm investing as $R_{i}^{2}(\theta)$. These expected payoffs are given by:

$$
\begin{aligned}
R_{E}^{1}(\theta) & =p(\theta) v_{E}(H)+(1-p(\theta)) v_{E}(L, \ell) \\
R_{E}^{2}(\theta) & =\frac{1}{2}\left[p(\theta) v_{E}(H)+(1-p(\theta)) v_{E}(L, \ell)\right] \\
R_{I}^{1}(\theta) & =p(\theta) v_{I}(H)+(1-p(\theta)) v_{I}(L, 0)-v_{I}(\ell, 0) \\
R_{I}^{2}(\theta) & =\frac{1}{2}\left[p(\theta) v_{I}(H)+(1-p(\theta))\left(v_{I}(L, 0)-v_{I}(\ell, L)\right)\right] .
\end{aligned}
$$

The critical projects are then given by $\theta_{i}^{k}: C\left(\theta_{i}^{k}\right)=R_{i}^{k}\left(\theta_{i}^{k}\right), i \in\{I, E\}, k \in\{1,2\}$. Since $p(\theta)$ is concave, so are all $R_{i}^{k}(\theta)$ (notice that all $R_{i}^{k}$ are concave monotone transformations of $p$ ). Given that $C$ is increasing and convex, all critical projects exist and are unique.

We first establish that the familiar ordering of critical values also holds in this setting.

Lemma B.9. Consider the case where the probability of drastic innovation $p:[0,1) \rightarrow$ $[0,1)$ is an increasing function of $\theta$.

(i) Under laissez-faire policy, the following relations hold:

(a) $\theta_{E}^{2}(A)=\theta_{I}^{2}(A) ;(b) \theta_{E}^{2}(A)<\theta_{E}^{1}(A)$.

(ii) Under no-acquisition policy, the following relations hold:

(a) $\theta_{E}^{2}(N)<\theta_{I}^{2}(N)$; (b) $\theta_{E}^{2}(N)<\theta_{E}^{1}(N)$.

(iii) In the killer-acquisition case, $\theta_{I}^{1}<\theta_{E}^{1}$ under both policies.

Proof. For part (a) in (i), note that $\forall \theta \in[0,1): R_{I}^{2}(\theta ; A)=R_{E}^{2}(\theta ; A)$ because $v_{I}^{A}(L, 0)-$ $v_{I}^{A}(\ell, L)=v_{E}^{A}(L, \ell)$ and $v_{I}^{A}(H)=v_{E}^{A}(H)$. Since $\theta_{I}^{2}(A)$ and $\theta_{E}^{2}(A)$ are implicitly given by $C\left(\theta_{I}^{2}(A)\right)=R_{I}^{2}\left(\theta_{I}^{2}(A) ; A\right)$ and $C\left(\theta_{E}^{2}(A)\right)=R_{E}^{2}\left(\theta_{E}^{2}(A) ; A\right)$ and both are unique, it directly follows that $\theta_{E}^{2}(A)=\theta_{I}^{2}(A)$. 
For part (a) in (ii), note that $\forall \theta \in[0,1): R_{I}^{2}(\theta ; N)>R_{E}^{2}(\theta ; N)$ because $v_{I}^{N}(L, 0)-$ $v_{I}^{N}(\ell, L)>v_{E}^{N}(L, \ell)$ by Assumption $1(\mathrm{iv})$ and $v_{I}^{N}(H)=v_{E}^{N}(H)$. Since $\theta_{I}^{2}(N)$ and $\theta_{E}^{2}(N)$ are implicitly given by $C\left(\theta_{I}^{2}(N)\right)=R_{I}^{2}\left(\theta_{I}^{2}(N) ; N\right)$ and $C\left(\theta_{E}^{2}(N)\right)=R_{E}^{2}\left(\theta_{E}^{2}(N) ; N\right)$, both are unique and $C$ is increasing, it directly follows that $\theta_{E}^{2}(N)<\theta_{I}^{2}(N)$.

For part (b) in both (i) and (ii), note that $\forall \theta \in[0,1)$ :

$$
\begin{aligned}
R_{E}^{1}(\theta) & =p(\theta) v_{E}(H)+(1-p(\theta)) v_{E}(L, \ell) \\
& >\frac{1}{2}\left[p(\theta) v_{E}(H)+(1-p(\theta)) v_{E}(L, \ell)\right]=R_{E}^{2}(\theta)
\end{aligned}
$$

Since $C$ is increasing and and the critical projects are unique, it directly follows that $\theta_{E}^{1}>\theta_{E}^{2}$.

Finally, for part (iii), note that $\forall \theta \in[0,1)$ :

$$
\begin{aligned}
R_{I}^{1}(\theta) & =p(\theta) v_{I}(H)+(1-p(\theta)) v_{I}(L, 0)-v_{I}(\ell, 0) \\
& =p(\theta)\left[v_{I}(H)-v_{I}(L, 0)\right] \\
& <p(\theta) v_{E}(H)+(1-p(\theta)) v_{E}(L, \ell)=R_{E}^{1}(\theta)
\end{aligned}
$$

where the second line follows from $v_{I}(L, 0)=v_{I}(\ell, 0)$ which is true in the killer-acquisition case irrespective of the policy. Moreover the following relations hold irrespective of the policy: $v_{I}(H)=v_{E}(H)$ by Assumption $1\left(\right.$ iii), $v_{E}(L, \ell) \geq \pi(L, \ell)-\kappa$ (where the inequality is strict under laissez-faire due to Assumption 1 (iv)) and $\pi(L, \ell)-\kappa \geq 0$ by Assumption 2(i). The strict inequality in the last line then follows by Assumption 1(i), $v_{I}(\ell, 0)>0$. Then, since $C$ is increasing and convex, it directly follows that $\theta_{I}^{1}<\theta_{E}^{1}$.

Since the orderings of critical projects don't change qualitatively, also the main result remains unchanged.

Proposition B.8. Consider the case where the probability of drastic innovation $p:[0,1) \rightarrow$ $[0,1)$ is an increasing function of $\theta$.

(i) In any equilibrium $\left(r_{I}^{N}, r_{E}^{N}\right)$ under the no-acquisition policy, (a) the variety of research projects is weakly smaller than in any equilibrium $\left(r_{I}^{A}, r_{E}^{A}\right)$ under the laissez-faire policy; and (b) the probability of any innovation is weakly smaller than in any simple equilibrium $\left(r_{I}^{A}, r_{I}^{A}\right)$ under the laissez-faire policy.

(ii) The inequalities in $(i)$ are strict, except if $\theta_{E}^{1}(A) \leq \theta_{E}^{1}(N)$.

Proof. Denote the equilibrium strategies under laissez-faire and the no-acquisition policy as $\left(r_{I}^{A}, r_{E}^{A}\right)$ and $\left(r_{I}^{N}, r_{E}^{N}\right)$, respectively. The result follows from Steps 1-5.

Step 1: $\mathcal{V}^{A}=\max \left\{\theta_{E}^{1}(A), \theta_{I}^{1}(A)\right\}$ and $\mathcal{V}^{N}=\max \left\{\theta_{E}^{1}(N), \theta_{I}^{1}(N)\right\}$.

Equipped with the ordering of critical projects we can apply Proposition A.1 to construct 
the equilibria with the small modification that mixed-strategy equilibria are given by the following expressions whenever they apply: $r_{E}(\theta)=\frac{R_{I}^{1}(\theta)-C(\theta)}{R_{I}^{1}(\theta)-R_{I}^{2}(\theta)}$ and $r_{I}(\theta)=\frac{R_{E}^{1}(\theta)-C(\theta)}{R_{E}^{1}(\theta)-R_{E}^{2}(\theta)}$. Hence, as before, $r_{I}^{A}(\theta)+r_{E}^{A}(\theta)=0$ if and only if $\theta \in\left(\max \left\{\theta_{E}^{1}(A), \theta_{I}^{1}(A)\right\}, 1\right)$ and $\mathcal{V}^{A}=$ $\max \left\{\theta_{E}^{1}(A), \theta_{I}^{1}(A)\right\}$. Similarly, the second claim holds because constructing equilibria using Proposition A.1, $r_{I}^{N}(\theta)+r_{E}^{N}(\theta)=0$ if and only if $\theta \in\left(\max \left\{\theta_{E}^{1}(N), \theta_{I}^{1}(N)\right\}, 1\right)$.

Step 2: $\theta_{I}^{1}(A)=\theta_{I}^{1}(N)$.

To show this, since critical projects are uniquely pinned down by $R_{I}^{1}\left(\theta_{I}^{1}\right)=C\left(\theta_{I}^{1}\right)$, it is sufficient that $R_{I}^{1}(\theta ; A)=R_{I}^{1}(\theta ; N) \forall \theta$, or equivalently

$$
p(\theta) v_{I}^{A}(H)+(1-p(\theta)) v_{I}^{A}(L, 0)-v_{I}^{A}(\ell, 0)=p(\theta) v_{I}^{N}(H)+(1-p(\theta)) v_{I}^{N}(L, 0)-v_{I}^{N}(\ell, 0)
$$

This holds since $v_{I}^{A}(t, 0)=v_{I}^{N}(t, 0)$ for all $t \in\{\ell, L, H\}$.

Step 3: $\theta_{E}^{1}(N)<\theta_{E}^{1}(A)$.

To show this, since $C$ is increasing, it is sufficient that $R_{E}^{1}(\theta ; N)<R_{E}^{1}(\theta ; A) \forall \theta$. The claim requires that $\forall \theta \in[0,1)$ :

$$
p(\theta) v_{E}^{N}(H)+(1-p(\theta)) v_{E}^{N}(L, \ell)<p(\theta) v_{E}^{A}(H)+(1-p(\theta)) v_{E}^{A}(L, \ell)
$$

This holds because $v_{E}^{N}(H)=v_{E}^{A}(H)$ and $v_{E}^{N}(L, \ell)<v_{E}^{A}(L, \ell)$, which is equivalent to

$$
\pi_{E}(L, \ell)-\kappa<\max \left\{\pi_{I}(L, 0)-\kappa, \pi_{I}(\ell, 0)\right\}-\pi_{I}(\ell, L)
$$

which holds by Assumption 1(iv).

Step 4: If $\theta_{E}^{1}(A)>\theta_{I}^{1}(A)$, then $\mathcal{V}^{A}>\mathcal{V}^{N}$ and if $\left(r_{I}^{A}, r_{E}^{A}\right)$ is a simple equilibrium then $\mathcal{P}\left(r_{I}^{A}, r_{E}^{A}\right)>\mathcal{P}\left(r_{I}^{N}, r_{E}^{N}\right)$.

Since $\theta_{E}^{1}(A)>\theta_{E}^{1}(N)$ by Step 3 and $\theta_{I}^{1}(A)=\theta_{I}^{1}(N)$ by Step 2, we obtain $\theta_{E}^{1}(A)>$ $\max \left\{\theta_{E}^{1}(N), \theta_{I}^{1}(N)\right\}$. Hence, $\mathcal{V}^{A}>\mathcal{V}^{N}$. Since $\mathcal{P}\left(r_{I}, r_{E}\right) \leq \mathcal{V}\left(r_{I}, r_{E}\right)$ for any $\left(r_{I}, r_{E}\right)$ and $\mathcal{P}\left(r_{I}, r_{E}\right)=\mathcal{V}\left(r_{I}, r_{E}\right)$ for simple equilibria, then also $\mathcal{P}\left(r_{I}^{A}, r_{E}^{A}\right)>\mathcal{P}\left(r_{I}^{N}, r_{E}^{N}\right)$ if $\left(r_{I}^{A}, r_{E}^{A}\right)$ is a simple equilibrium.

Step 5: If $\theta_{E}^{1}(A) \leq \theta_{I}^{1}(A)$, then $\mathcal{V}^{A}=\mathcal{V}^{N}$ and if $\left(r_{I}^{A}, r_{E}^{A}\right)$ is a simple equilibrium then $\mathcal{P}\left(r_{I}^{A}, r_{E}^{A}\right) \geq \mathcal{P}\left(r_{I}^{N}, r_{E}^{N}\right)$.

If $\theta_{E}^{1}(A) \leq \theta_{I}^{1}(A)$, then by Steps 2 and $3, \theta_{E}^{1}(N)<\theta_{I}^{1}(N)$. Then $\mathcal{V}^{A}=\theta_{I}^{1}(A)=\theta_{I}^{1}(N)=$ $\mathcal{V}^{N}$. Since $\mathcal{P}\left(r_{I}, r_{E}\right) \leq \mathcal{V}\left(r_{I}, r_{E}\right)$ for any $\left(r_{I}, r_{E}\right)$ and $\mathcal{P}\left(r_{I}, r_{E}\right)=\mathcal{V}\left(r_{I}, r_{E}\right)$ for simple equilibria, then also $\mathcal{P}\left(r_{I}^{A}, r_{E}^{A}\right) \geq \mathcal{P}\left(r_{I}^{N}, r_{E}^{N}\right)$.

While our results on banning acquisitions on any innovation is qualitatively robust, there is an important twist: Banning acquisitions will lead to a reduction in investment into the most expensive projects (if it leads to a reduction in investment). Since the most expensive projects are those with the highest probability of drastic innovation, giving those 
up disproportionally reduces the probability of drastic innovation relative to non-drastic innovation.

\section{B.6 Consumer Surplus Effects}

We now ask under which circumstances the positive competition effect of prohibiting acquisitions dominates the negative innovation effect from a consumer perspective, focusing on the killer-acquisition case. ${ }^{36}$ We provide the main results in Section B.6.1. Section B.6.2 gives the details for the parameterized examples.

\section{B.6.1 Main Results}

We denote consumer surplus when the entrant competes with technology $L$ against the incumbent as $S(\ell, L)$, and as $S(t)$ for a monopoly with technology $t \in\{\ell, H\} .37$ We assume that $S(H)>S(\ell, L)>S(\ell)$. Thus, consumers prefer the high-state monopoly to the duopoly, which they prefer to the low-state monopoly in turn. We denote the probability of a duopoly in policy regime $R$ as $\operatorname{prob}^{R}(\ell, L)$ and the probability of a monopoly with technology $t \in\{\ell, H\}$ as $\operatorname{prob}^{R}(t) .{ }^{38}$ Then, the expected consumer surplus under laissezfaire is:

$$
\operatorname{prob}^{A}(H) S(H)+\operatorname{prob}^{A}(\ell) S(\ell) .
$$

Under the no-acquisition policy, the expected consumer surplus is:

$$
\operatorname{prob}^{N}(H) S(H)+\operatorname{prob}^{N}(\ell, L) S(\ell, L)+\operatorname{prob}^{N}(\ell) S(\ell) .
$$

The following result gives a simple condition under which the competition effect dominates the innovation effect from a consumer perspective.

Proposition B.9. Suppose the killer-acquisition case applies. Prohibiting start-up acquisitions increases the expected consumer surplus if and only if

$$
\operatorname{prob}^{N}(\ell, L)[S(\ell, L)-S(\ell)]>\left[\operatorname{prob}^{A}(H)-\operatorname{prob}^{N}(H)\right][S(H)-S(\ell)]
$$

\footnotetext{
${ }^{36}$ In the genuine-acquisition case, such an analysis is not necessary for $\theta_{E}^{1}(A) \leq \theta_{I}^{1}(A)$, because then there is no innovation effect by Proposition 2. If $\theta_{E}^{1}(A)>\theta_{I}^{1}(A)$, the analysis and the insights for the genuine- and killer-acquisition cases are similar. However, since the decomposition of the welfare effect is more involved in the former case, we focus on the killer-acquisition case.

${ }^{37}$ Note that, while only the incumbent can be a monopolist with technology $\ell$, both incumbent and entrant may end up with an $H$ monopoly in both regimes.

${ }^{38}$ Note that these probabilities follow directly from the equilibrium innovation strategies $\left(r_{I}, r_{E}\right)$, characterized in Propositions 1, B.1 and B.2.
} 
Proof. Subtracting the two expressions for expected consumer surplus gives the welfare difference

$$
\begin{array}{r}
\operatorname{prob}^{N}(\ell, L) S(\ell, L)+\left[\operatorname{prob}^{N}(H)-\operatorname{prob}^{A}(H)\right] S(H)+ \\
{\left[\operatorname{prob}^{N}(\ell)-\operatorname{prob}^{A}(\ell)\right] S(\ell)=} \\
\operatorname{prob}^{N}(\ell, L)[S(\ell, L)-S(\ell)]+\left[\operatorname{prob}^{N}(H)-\operatorname{prob}^{A}(H)\right] S(H)+ \\
{\left[\operatorname{prob}^{N}(\ell)+\operatorname{prob}^{N}(\ell, L)-\operatorname{prob}^{A}(\ell)\right] S(\ell)}
\end{array}
$$

The result then follows because

$$
\operatorname{prob}^{N}(\ell)+\operatorname{prob}^{N}(\ell, L)-\operatorname{prob}^{A}(\ell)=\operatorname{prob}^{A}(H)-\operatorname{prob}^{N}(H) .
$$

The proposition illustrates the countervailing effects of prohibiting acquisitions. On the one hand, the policy measure introduces desirable competition (and potentially better technology) with probability $\operatorname{prob}^{N}(\ell, L)$, leading to a competitive surplus $S(\ell, L)$ rather than the non-competitive surplus $S(\ell)$. On the other hand, the measure reduces the probability of a drastic innovation (which would increase consumer surplus from $S(\ell)$ to $S(H))$ by $\operatorname{prob}^{A}(H)-\operatorname{prob}^{N}(H)$. Note that $S(H)-S(\ell)$ depends on the size of the drastic innovation and, closely related, on its effect on demand, whereas $S(\ell, L)-S(\ell)$ captures the consumer value of duopolistic competition. Both terms are independent of the firms' investment decisions. By contrast, $\operatorname{prob}^{N}(\ell, L)$ is the product of the entrant's endogenous innovation probability under the no-acquisition policy and the conditional probability $1-p$ that this innovation is non-drastic. $\operatorname{prob}^{A}(H)-\operatorname{prob}^{N}(H)$ is the product of the effect of the acquisition policy on the probability of an innovation success (see Section 4) and the conditional probability $p$ that an innovation is drastic.

These general considerations lead to some insights into the determinants of the consumer surplus effect. Assuming that the effect on probability corresponds to the effect on variety (see the discussion of Proposition 2(b)), an increase in the entrant's bargaining power $\beta$ increases $\operatorname{prob}^{A}(H)-\operatorname{prob}^{N}(H)$ and thus the adverse innovation effect of a restrictive acquisition policy; there is no such effect when $\beta=0 .{ }^{39}$ Therefore, a restrictive acquisition policy will always be justified for sufficiently low bargaining power of the entrant, but not necessarily when this bargaining power increases.

By contrast, whether prohibiting acquisitions increases or decreases consumer surplus depends on product market competition in an ambiguous way. According to Proposition 3 , in the killer-acquisition case an exogenous reduction in the entrant's duopoly profits

\footnotetext{
${ }^{39}$ Remember that the extent to which the policy induces desirable competition only depends on the entrant's innovation probability under no-acquisition, which is independent of $\beta$.
} 


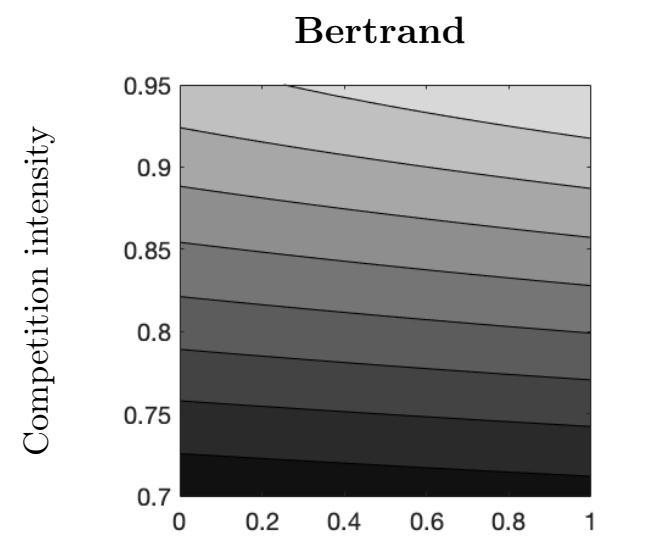

Bargaining power of the entrant

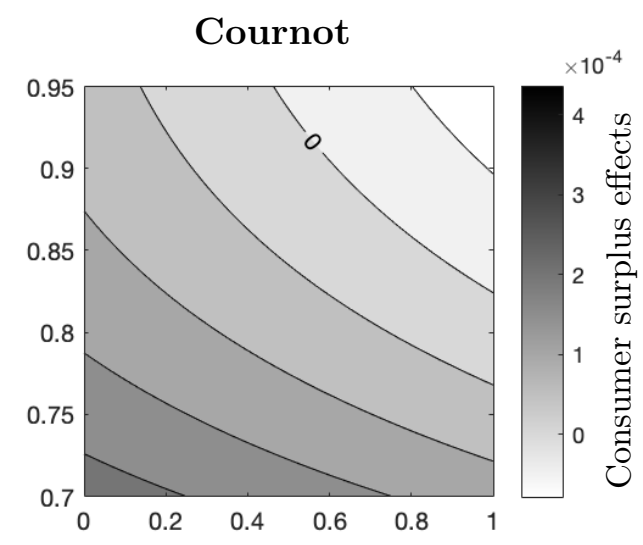

Bargaining power of the entrant

Figure 3: Effect of prohibiting acquisitions on consumer surplus: Contour plot depicts effect levels when firms compete à la heterogenous Bertrand (left panel) or heterogenous Cournot (right panel) based on a parameterized example as described in Section B.6.2. Brighter shade implies lower effect and the "0" highlights the no effect contour line.

$\pi_{E}(L, \ell)$ tends to increase the size of the adverse innovation effect. However, such a change in the market environment may reflect more intense competitive interaction between the firms and therefore a higher consumer surplus $S(\ell, L)$ relative to the monopoly case. Thus whether a reduction in the entrant's duopoly profits makes a positive consumer surplus effect of prohibiting acquisitions more or less likely is not clear without considering special parameterized models. Similar arguments apply to the incumbent's duopoly profits.

We analyze these ambiguities in a standard linear demand model, where we consider heterogeneous Bertrand as well as heterogeneous Cournot competition. Figure 3 shows that the overall effect of prohibiting acquisitions on consumer surplus is in line with our comparative statics result for the killer-acquisition case (see Proposition 3): The lighter shades towards the top right corner of both panels represent the fact that the prohibition of acquisitions has a monotonously lower effect on consumer surplus the higher the bargaining power and intensity of competition, which is true for both modes of competition. ${ }^{40}$ Whether prohibiting acquisitions may even result in a negative consumer-surplus effect depends on the specific parameterization and mode of competition. In the left panel of Figure 3 with Bertrand competition, the innovation effect never fully trumps the competition effect. However, when firms compete à la Cournot as is the case in the right panel of Figure 3, the effect of prohibition actually turns negative for parameter combinations in the north east of the zero effect contour line.

Our focus on consumer surplus in this welfare discussion reflects the common practice

\footnotetext{
${ }^{40}$ Here, the intensity of competition corresponds to the degree of substitution between the goods, with higher intensity (i.e. higher substitutability between goods) leading to lower duopoly profits. The details of the model and our calculations can be found in the following Section B.6.2.
} 
of many competition agencies. That said, extending the analysis beyond this welfare standard may well be interesting. For instance, the discussion of duplication in Section 5.3 suggests further channels by which the acquisition policy can affect welfare.

\section{B.6.2 Calculations for Consumer Surplus Effects in Figure 3}

Product Market We assume linear demand and consider both heterogeneous Bertrand as well as Cournot competition. The utility of the representative consumer is given by:

$$
U\left(q_{I}, q_{E}\right)=\alpha_{I} q_{I}+\alpha_{E} q_{E}-\frac{1}{2}\left[\left(q_{I}^{2}+q_{E}^{2}\right)+2 \gamma q_{I} q_{E}\right]
$$

where $q_{i}$ is the quantity consumed from firm $i \in\{I, E\}, \alpha_{i}$ is a quality parameter and $\gamma$ governs substitutability. If $\gamma=0$, both products are independent. When both firms are active, the demand functions are:

$$
q_{i}\left(p_{i}, p_{j}\right)=\frac{\alpha_{i}-\alpha_{j} \gamma-p_{i}+\gamma p_{j}}{1-\gamma^{2}}
$$

We normalize marginal cost of production to 0 , hence we focus on product innovations which may increase the quality parameter $\alpha_{i}$. The quality of the incumbent's product is $\alpha_{L} \in \mathbb{R}^{+}$, which is also the quality of the entrant's product under a non-drastic innovation, i.e. $L=\ell$. The minimum quality level of a drastic product innovation is then given by the condition that, even if the firm owning the drastic technology $\alpha_{H}$ sets a monopoly price, the rival firm cannot profitably compete in the market, which can be derived as $\alpha_{H} \geq \frac{2}{\gamma} \alpha_{L}$.

Assumptions 1(i), (ii) and (iii) are satisfied by construction. For suitable parameter spaces 1 (iv) and 2 are satisfied as well.

Innovation Effect We assume that $C(\theta)=\frac{s \theta}{1-\theta}$ (where $s>0$ ) to calculate the equilibrium investments. Remember that for some critical value constellations, equilibria are not unique. Therefore, we calculate bounds on the innovation and competition effects. Using Proposition 1 and denoting equilibrium intermediate effort levels with $r_{E}^{\mu}(\theta)=$ $\frac{C\left(\theta_{I}^{1}(\mu)\right)-C(\theta)}{C\left(\theta_{I}^{1}(\mu)\right)-C\left(\theta_{I}^{2}(\mu)\right)}$ and $r_{I}^{\mu}(\theta)=\frac{C\left(\theta_{E}^{1}(\mu)\right)-C(\theta)}{C\left(\theta_{E}^{1}(\mu)\right)-C\left(\theta_{E}^{2}(\mu)\right)}$, where $\mu \in\{A, N\}$, the upper and lower bound innovation probabilities in the laissez-faire regime are

$$
\begin{aligned}
\overline{\operatorname{prob}^{A}(H)} / p & =\theta_{E}^{1}(A) \\
\underline{\operatorname{prob}^{A}(H)} / p & =\theta_{E}^{1}(A)-\max \left\{\theta_{I}^{1}(A)-\theta^{2}(A), 0\right\} \\
& +\max \left\{\int_{\theta^{2}}^{\theta_{I}^{1}} r_{E}^{A}+r_{I}^{A}-r_{E}^{A} r_{I}^{A} d \theta, 0\right\} .
\end{aligned}
$$


Using Proposition B.1, the upper and lower bound in the no-acquisition regime are:

$$
\begin{aligned}
\overline{\operatorname{prob}^{N}(H)} / p & =\theta_{E}^{1}(N) \\
& -\max \left\{\min \left\{\theta_{I}^{2}(N), \theta_{E}^{1}(N)\right\}-\max \left\{\theta_{I}^{1}(N), \theta_{E}^{2}(N)\right\}, 0\right\} \\
& +\max \left\{\int_{\max \left\{\theta_{I}^{1}, \theta_{E}^{2}\right\}}^{\min \left\{\theta_{I}^{2}, \theta_{E}^{1}\right\}} r_{E}^{N}+r_{I}^{N}-r_{E}^{N} r_{I}^{N} d \theta, 0\right\} \\
\underline{\operatorname{prob}^{N}(H) / p} & =\theta_{E}^{1}(N) \\
& -\left|\min \left\{\theta_{I}^{2}(N), \theta_{E}^{1}(N)\right\}-\max \left\{\theta_{I}^{1}(N), \theta_{E}^{2}(N)\right\}\right| \\
& +\left|\int_{\max \left\{\theta_{I}^{1}, \theta_{E}^{2}\right\}}^{\min \left\{\theta_{I}^{2}, \theta_{E}^{1}\right\}} r_{E}^{N}+r_{I}^{N}-r_{E}^{N} r_{I}^{N} d \theta\right| .
\end{aligned}
$$

We obtain the upper bound on the effect on drastic innovation by selecting equilibria in the two regimes, such that the policy has the least negative effect on the probability of drastic innovation, which is $\overline{\operatorname{prob}^{N}(H)}-\underline{\operatorname{prob}^{A}(H)}$. Similarly, the lower bound is $\underline{\operatorname{prob}^{N}(H)}-$ $\overline{\operatorname{prob}^{A}(H)}$.

Competition Effect The competition effect is given by the reduction in the entry probability. Since there is no competition in the laissez-faire regime, we only need to consider the probability of an $L$ innovation by the entrant in the no-acquisition regime. We again calculate upper and lower bounds using Proposition B.1:

$$
\begin{aligned}
\overline{\operatorname{prob}^{N}(L, L)} /(1-p) & =\frac{1}{2} \theta_{E}^{2}(N)+\theta_{E}^{1}(N)-\min \left\{\theta_{I}^{2}(N), \theta_{E}^{1}(N)\right\} \\
& +\max \left\{\int_{\max \left\{\theta_{E}^{2}(N), \theta_{I}^{1}(N)\right\}}^{\min \left\{\theta_{I}^{2}(N), \theta_{E}^{1}(N)\right\}} r_{E}^{N}\left(1-r_{I}^{N}\right)+\frac{1}{2} r_{E}^{N} r_{I}^{N} d \theta, 0\right\} \\
\underline{\operatorname{prob}^{N}(L, L)} /(1-p) & =\frac{1}{2} \theta_{E}^{2}(N)+\theta_{E}^{1}(N)-\min \left\{\max \left\{\theta_{I}^{1}(N), \theta_{I}^{2}(N)\right\}, \theta_{E}^{1}(N)\right\} \\
& +\max \left\{\int_{\max \left\{\theta_{E}^{2}(N), \theta_{I}^{1}(N)\right\}}^{\min \left\{\theta_{I}^{2}(N), \theta_{E}^{1}(N)\right\}} r_{E}^{N}\left(1-r_{I}^{N}\right)+\frac{1}{2} r_{E}^{N} r_{I}^{N} d \theta, 0\right\} .
\end{aligned}
$$

Overall Consumer Surplus Effect Note that consumer surplus differences $S(L, L)$ $S(L)$ and $S(H)-S(L)$ are calculated by the net utility difference of the representative consumer for the respective technological states of the firms. The upper bound on the consumer surplus effect $\overline{\Delta S}$ represents the effect of banning acquisitions when selecting equilibria which are most preferable to the policy change, thus considering the upper bound on the competition and the innovation effect; vice versa for the lower bound on the consumer surplus effect $\underline{\Delta S}$ (see Proposition B.9):

$$
\overline{\Delta S}=\overline{\operatorname{prob}^{N}(L, L)}[S(L, L)-S(L)]+\left(\overline{\operatorname{prob}^{N}(H)}-\underline{\left.\operatorname{prob}^{A}(H)\right)}[S(H)-S(L)]\right.
$$




$$
\underline{\Delta S}=\underline{\operatorname{prob}^{N}(L, L)}[S(L, L)-S(L)]+\left(\underline{\operatorname{prob}^{N}(H)}-\overline{\operatorname{prob}^{A}(H)}\right)[S(H)-S(L)] .
$$

Parameter Values Figure 3 is constructed considering the following values for the quality parameters: $a_{L}=0.5, a_{H}=1.5$. The probability of a drastic innovation is chosen at $p=0.01$, such that consumer surplus effects are unique, that is effects where $\overline{\Delta S}=\Delta S$. Other parameters differ by mode of competition to bring the depicted effects on a similar level. The scaling parameter in the investment cost function is taken to be $s=2(s=0.5)$ and the commercialization costs are given by $\kappa=0.005(k=0.028)$ in case of Bertrand (Cournot) competition. We consider $\gamma \geq 0.70$ to make sure Assumption 1(iv) is satisfied and $\gamma \leq 0.95$ to make sure Assumption 2 is satisfied. Hence, Figure 3 is depicted in the parameter space $\gamma \in[0.70,0.95]$ and $\beta \in[0,1]$.

\section{B.7 One-dimensional Innovation Model}

In this section we show that, in a model where firms only choose the amount of resources they invest in research, banning acquisitions will have an ambiguous effect on innovations.

Let $x_{i}$ be the probability that the firm $i \in\{I, E\}$ discovers the innovation, with the associated cost given by $K(\cdot)$, where $K$ is strictly increasing and convex. Apart from the investment stage, the model is unchanged.

Profits and Best Responses The expected profit of the incumbent and the entrant, given $x_{I}$ and $x_{E}$, can be written as

$$
\begin{aligned}
\mathbb{E} \Pi_{I}\left(x_{I}, x_{E}\right)= & x_{I}\left(1-\frac{1}{2} x_{E}\right)\left[p v_{I}(H)+(1-p) v_{I}(L, 0)\right] \\
& +x_{E}\left(1-\frac{1}{2} x_{I}\right)(1-p) v_{I}(\ell, L)+\left(1-x_{I}\right)\left(1-x_{E}\right) v_{I}(\ell, 0)-K\left(x_{I}\right) \\
\mathbb{E} \Pi_{E}\left(x_{E}, x_{I}\right)= & x_{E}\left(1-\frac{1}{2} x_{I}\right)\left[p v_{E}(H)+(1-p) v_{E}(L, \ell)\right]-K\left(x_{E}\right) .
\end{aligned}
$$

Consequently, the first-order conditions and, implicitly, the best responses of the firms are

$$
\begin{aligned}
& K^{\prime}\left(x_{I}\left(x_{E}\right)\right)=\left(1-x_{E}\right)\left[p v_{I}(H)+(1-p) v_{I}(L, 0)-v_{I}(\ell, 0)\right] \\
&+\frac{1}{2} x_{E}\left[p v_{I}(H)+(1-p)\left(v_{I}(L, 0)-v_{I}(\ell, L)\right)\right] \\
& K^{\prime}\left(x_{E}\left(x_{I}\right)\right)=\left(1-\frac{1}{2} x_{I}\right)\left[p v_{E}(H)+(1-p) v_{E}(L, \ell)\right]
\end{aligned}
$$

The Nash equilibrium solves the above system of equations and is denoted by $\left(x_{I}^{*}, x_{E}^{*}\right){ }^{41}$

Note that the values $v_{I}\left(t_{I}^{i n t}, t_{E}^{i n t}\right)$ and $v_{E}\left(t_{E}^{i n t}, t_{I}^{\text {int }}\right)$ are exactly the same as in the main model and thus given by Lemma 2 for the laissez-faire regime. If acquisitions are prohib-

\footnotetext{
${ }^{41}$ Second order conditions are satisfied due to convexity of $K(x)$.
} 
ited, the only terms changing in the above first-order conditions are $v_{I}(\ell, L)$ and $v_{E}(L, \ell)$. Thus, we use superscripts to disentangle the different regimes: $v_{I}^{A}(\ell, L)$ and $v_{E}^{A}(L, \ell)$ in laissez-faire and $v_{I}^{N}(\ell, L)$ and $v_{E}^{N}(L, \ell)$ in the no-acquisition policy, where $v_{I}^{N}(\ell, L)=\pi(\ell, L)$ and $v_{E}^{N}(L, \ell)=\pi(L, \ell)-\kappa$.

If acquisitions are prohibited, the incumbent's payoff is lower when the entrant discovers an innovation (compared to the case when acquisitions are allowed), increasing her incentives to invest into R\&D in order to drive out the entrant. However, the entrant also receives lower profits when he obtains a non-drastic innovation, which reduces his overall innovation incentives. Due to these counteracting effects, the net effect of a ban on acquisitions on the sum of investment levels is not clear ex-ante.

Effect of Acquisitions on Innovation Probability We assume $\pi(\ell, 0)>\pi(L, 0)-$ $\kappa$, so that $v_{I}(L, 0)=v_{I}(\ell, 0)$. To simplify the comparison between policy regimes, we introduce a new parameter $\mu$, where $\mu$ represents the probability that the acquisition will be allowed. The first order conditions of the entrant and incumbent for a given regime $\mu$ are given by:

$$
\begin{aligned}
K^{\prime}\left(x_{I}\left(x_{E}\right) ; \mu\right)= & \left(1-x_{E}\right) p\left(v_{I}(H)-v_{I}(\ell, 0)\right) \\
& +\frac{1}{2} x_{E}\left(p v_{I}(H)+(1-p)\left[\mu v_{E}^{A}(L, \ell)+(1-\mu)\left(v_{I}(\ell, 0)-v_{I}^{N}(\ell, L)\right)\right]\right) \\
K^{\prime}\left(x_{E}\left(x_{I}\right) ; \mu\right)= & \left(1-\frac{1}{2} x_{I}\right)\left(p v_{E}(H)+(1-p)\left[\mu v_{E}^{A}(L, \ell)+(1-\mu) v_{E}^{N}(L, \ell)\right]\right) .
\end{aligned}
$$

The probability of an innovation, and its change when $\mu$ increases are given by:

$$
\begin{aligned}
\operatorname{Pr}(\text { Innovation }) & =x_{I}^{*}(\mu)+x_{E}^{*}(\mu)-x_{I}^{*}(\mu) x_{E}^{*}(\mu) \\
\Rightarrow \frac{d \operatorname{Pr}(\text { Innovation })}{d \mu} & =\left(1-x_{E}^{*}(\mu)\right) \frac{d x_{I}^{*}(\mu)}{d \mu}+\left(1-x_{I}^{*}(\mu)\right) \frac{d x_{E}^{*}(\mu)}{d \mu} .
\end{aligned}
$$

We use the implicit function theorem on the first order conditions of the incumbent and entrant to evaluate the effect on the innovation efforts, $\frac{d x_{I}^{*}(\mu)}{d \mu}$ and $\frac{d x_{E}^{*}(\mu)}{d \mu}$. Inserting these expressions into the above derivative of the innovation probability, we get:

$$
\begin{aligned}
\frac{d \operatorname{Pr}(\text { Innovation })}{d \mu} & =\frac{\frac{1}{2} x_{E}^{*}(\mu)(1-p)\left(v_{I}^{A}(\ell, L)-v_{I}^{N}(\ell, L)\right) * \mathcal{I}}{|J|} \\
& +\frac{\left(1-\frac{1}{2} x_{I}^{*}(\mu)\right)(1-p)\left(v_{E}^{A}(L, \ell)-v_{E}^{N}(L, \ell)\right) * \mathcal{E}}{|J|}
\end{aligned}
$$

where

$$
\mathcal{I}=\frac{1}{2}\left(1-x_{I}^{*}(\mu)\right)\left(p v_{E}(H)+(1-p)\left(\mu v_{E}^{A}(L, \ell)+(1-\mu) v_{E}^{N}(L, \ell)\right)\right)
$$




$$
-\left(1-x_{E}^{*}(\mu)\right) K^{\prime \prime}\left(x_{E}^{*}(\mu)\right)
$$

and

$$
\begin{aligned}
\mathcal{E} & =\frac{1}{2}\left(1-x_{E}^{*}(\mu)\right)\left[p v_{I}(H)+(1-p)\left(v_{I}(\ell, 0)-\mu v_{I}^{A}(\ell, L)-(1-\mu) v_{I}^{N}(\ell, L)\right)\right. \\
& \left.-2 p\left(v_{I}(H)-v_{I}(\ell, 0)\right)\right]+\left(1-x_{I}^{*}(\mu)\right) K^{\prime \prime}\left(x_{I}^{*}(\mu)\right) .
\end{aligned}
$$

Note that the Jacobian matrix $J$ is the collection of second-order partial derivatives and is negative definite assuming strict convexity of the cost function $K(x)$. Hence the determinant of the Jacobian matrix $|J|$ is positive and the sign of the effect of acquisitions on innovation probability is the same as the sign of weighted sum of $\mathcal{I}$ and $\mathcal{E}$.

This sign is not clear ex-ante. If $\beta=0$, so that $v_{E}^{A}(L, \ell)=v_{E}^{N}(L, \ell)$, then the sign of the effect on innovation probability is determined by

$$
\left.\frac{d P r(\text { Innovation })}{d \mu}\right|_{\beta=0} \gtrless 0 \Leftrightarrow\left(p v_{E}(H)+(1-p) v_{E}^{N}(L, \ell)\right) \gtrless 2 \frac{\left(1-x_{E}^{*}(\mu)\right) K^{\prime \prime}\left(x_{E}^{*}(\mu)\right)}{1-x_{I}^{*}(\mu)} \text {. }
$$

This effect is likely to be negative for large competition intensity in a duopoly, i.e. relatively small $\pi(L, \ell)=v_{E}^{N}(L, \ell)+\kappa$.

If the entrant has all bargaining power, i.e. $\beta=1$ and $v_{I}^{A}(\ell, L)=v_{I}^{N}(\ell, L)$, we get a similar expression for the sign of the effect:

$$
\begin{aligned}
& \left.\frac{d \operatorname{Pr}(\text { Innovation })}{d \mu}\right|_{\beta=1} \gtrless 0 \\
& \quad \Leftrightarrow(1-p)\left(v_{I}(\ell, 0)-v_{I}^{N}(\ell, L)\right)+p\left(2 v_{I}(\ell, 0)-v_{I}(H)\right) \gtrless-2 \frac{\left(1-x_{I}^{*}(\mu)\right) K^{\prime \prime}\left(x_{I}^{*}(\mu)\right)}{1-x_{E}^{*}(\mu)}
\end{aligned}
$$

If drastic innovation is not too profitable, i.e. $v_{I}(H)<2 v_{I}(\ell, 0)$, a more lenient regime towards acquisitions will increase innovation probability, irrespective of product market competition intensity when both firms are active.

The above analysis shows that, if firms cannot target their R\&D efforts towards specific projects, the innovation effect of a more restrictive policy towards acquisition of start-ups will be ambiguous in general. 